\title{
UTILIZATION-FOCUSED EVALUATION OF THE PROGRAM EVALUATION PROCESS OF A MISSOURI SCHOOL DISTRICT
}

\author{
A Dissertation \\ presented to \\ The faculty of the Graduate School \\ at the University of Missouri-Columbia \\ In Partial Fulfillment \\ of the Requirements for the Degree \\ Doctor of Education \\ by \\ LAURA NELSON \\ Dr. Joyce Piveral and Dr. Phillip Messner, Dissertation Supervisors
}

DECEMBER 2008 
(C) Copyright by Laura Nelson 2008

All Rights Reserved 
The undersigned, appointed by the Dean of the Graduate School, have examined the dissertation entitled

\section{UTILIZATION-FOCUSED EVALUATION OF THE PROGRAM EVALUATION PROCESS OF A MISSOURI SCHOOL DISTRICT}

Presented by Laura Nelson

A candidate for the degree of Doctor of Education

And hereby certify that in their opinion it is worthy of acceptance.

Dr. Joyce Piveral

Dr. Phillip Messner

Dr. Michael Graham

Dr. Max Fridell

Dr. Margaret Drew 


\section{DEDICATION}

For Tom, Lindsey, Ridge, \& Kit 


\section{ACKNOWLEDGEMENTS}

The lessons I have learned and the gratitude I owe can be expressed in terms of four emergent themes: strength within, just push the button, the importance of the collective mind, and love and acceptance. Together these themes inform my understanding of the Japense word kansha, a term for what it means to have deep gratitude for the role other people play in one's life.

Strength within. The personification of strength within is my husband, Tom. He has been the guiding force behind this entire endeavor and the ultimate role model for living a tenacious, exhilarating life. For everything I didn't think I could do, he had five or more reasons why I could. His insistence that I was more that I thought I was, made it so. Tom, thank you for making every day of my life wonderful and for ensuring that this program of study came to fruition. The dissertation is as much yours as it is mine.

Just push the button. A second significant theme is embodied by the words "just push the button" (M. Smith, multiple personal communications) and their analogues "just Fedex the application" (J.Westfall, personal communication December, 2004) and “enough is enough" (Smith and Westfall, multiple personal communications). Dr. Melody Smith, thank you for being my mentor, my leader, my boss, and my friend, for inspiring not only this study but a vision for the future of our profession, for standing by me, and for ensuring that I value learning more than being right. Dr. Jeanette Westfall, thank you for refusing to leave me behind, for all of the knowledge creation we managed on our marathon rides back and forth to MU, for constantly helping me frame and reframe all of the "opportunities" that have come along these past few years, and for your steadfast friendship. Mel and JW-I owe you both a debt of gratitude beyond measure. 
The importance of the collective mind. It was through ELPA I learned the truth of this third theme. My professors and colleagues have all helped instill the requisite understanding that collaboration really does make a significant positive impact on the world and on individuals, particularly me. I owe a special thank you to my dissertation advisors, Dr. Joyce Piveral and Dr. Phil Messner. You have shifted my paradigm for thinking about learning and leading and have enabled me to become more expansive in my ability to do both. I am not only a better practitioner for having been guided by you, but I am a better person as well. Dr. Piveral, you have been a blessing in my professional life since my first year at Central and a seminal influence upon the paths I have chosen thank you for so many years of guidance. I am also grateful to the other members of my committee for the time you spent helping me refine this research. Dr. Margaret Drew, Dr. Max Fridell, Dr. Michael Graham, and again, Dr. Piveral, and Dr. Messner, thank you all for your expertise and the generosity with which you shared it with me! Additionally, I am grateful for the instructional teams at MU, particularly Dr. Joe Donaldson and Dr. Margaret Grogan, from whom I learned so very much over the course of this program.

This theme also is pertinent to the thanks I owe my colleagues in the St. Joseph School District. To the members of the Superintendent's Cabinet- thank you for the support, wisdom, guidance, and patience you have extended to me during this process. Mrs. Marlie Williams and Dr. Jaime Dial, a special thanks to you for brownies, persistent reality checks, and friendship. I owe a significant debt of particular gratitude to my assessment office colleagues. Melody Boring - thank you for all you have taught me professionally and personally. You have helped me in more ways than you know. Kerry Shepherd, you have made me laugh, inspired me, kept me on track, modeled 
fearlessness, and reminded me of what does and does not work - for this and more, thank you.

Love and acceptance. The final theme is coded as love and acceptance and directly related to the importance of my dear family and friends, without whom I especially would not be the person I am. Thank you all for accepting and supporting the havoc that this commitment on my part created in your lives. To my parents and my parents-in-law (Gordon and Thea Ide, Patrick and Susie Thomas, and Robert and Susie Nelson) thank you so much for all you do and for being the role models you are to me. To my sisters and sisters-in-law (Steph, Ali, Whit, Anita, Rhonda, and Nora) and my brothers and brothers-in-law (Andy, Doug, Trey, Craig, Bob, Ron, and John) thank you for constantly being there and reminding me of what matters in life. To my friends thank you for sticking by me and for all the encouragement, not to mention prayers, cards, calls, coffee, and candy you sent along the way. To my darling children, Kit, Ridge, and Lindsey- I am so very proud of you and grateful for all the slack you cut me these past few years. You are the ultimate source of joy, inspiration, and wonder. Your senses of humor and intelligence inform my worldview and continuously impact the meaning that I am able to make of life, both generally and specifically. Tom, thank you for the fact that you literally ran our entire household, stem to stern, during this process, for patiently doing all the things I couldn't, and for making me get this done. I love you beyond measure.

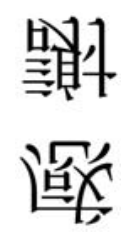

Kansha 
TABLE OF CONTENTS

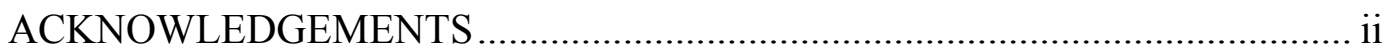

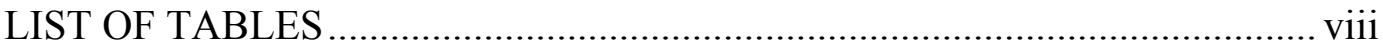

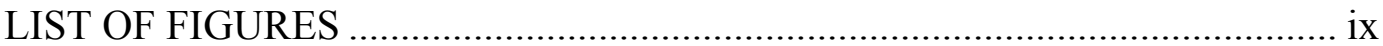

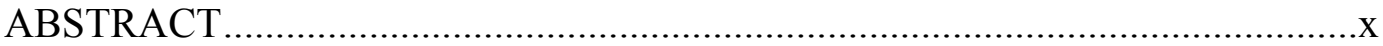

CHAPTER

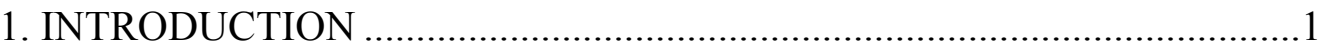

Conceptual Underpinnings of the Study

Statement of the Problem

Purpose of the Study

Research Questions

Methodology

Significance of the Study

Definition of Terms

Delimitations

Limitations

Summary

2. REVIEW OF RELATED LITERATURE ……………..............................16

3. RESEARCH DESIGN AND METHODOLOGY ……...................................45

Introduction

Research Paradigm and Philosophy

Problem and Purpose Overview 


Research Questions
Research Methods
Setting and Population
Instrument
Data Collection Strategies
Data Analysis
Summary

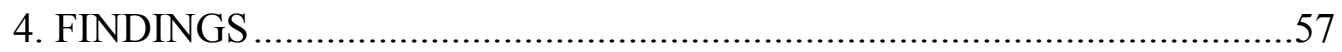

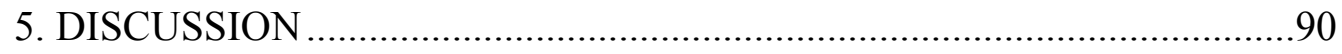

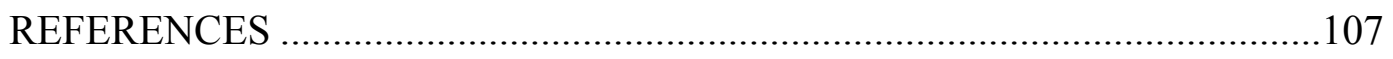

\section{APPENDICES}

A: 26 Evaluation Approaches ...................................................................113

B: Request for Permission to Conduct Research ............................................115

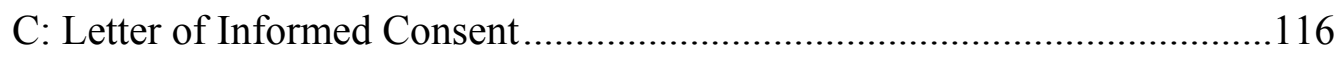

D: First Long Interview Protocol ................................................................119

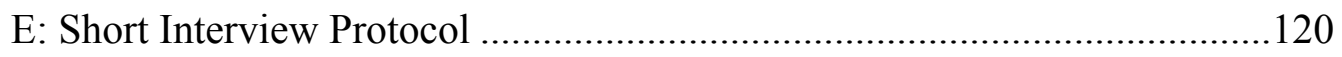

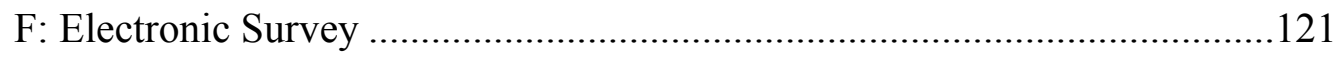

G: Second Long Interview Protocol ..............................................................122

H: Electronic Survey Cover Letter ............................................................123

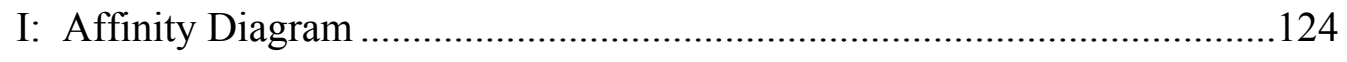

J: MSIP, Cycle 4 Content Analysis Diagram..................................................125

K: NCLB Content Analysis Diagram ........................................................126

L: School District Board Policy Content Analysis Diagram ...........................127 
M: Vision, Mission, Goals, and Values Content Analysis Diagram ..............128

$\mathrm{N}$ : Example of NCLB Document Analysis................................................129

O: Examples of Programs - Data Presented to Primary Intended User ..........130

P: Example of Coded Transcript ............................................................131

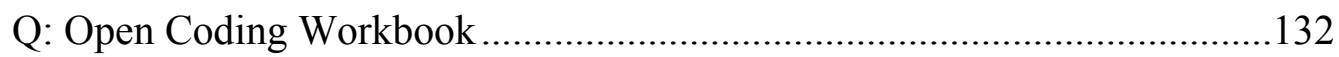

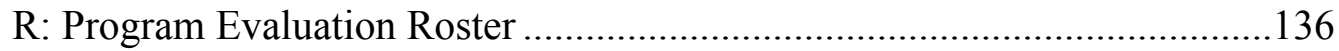

S: Program Evaluation Commissioning Form ............................................137

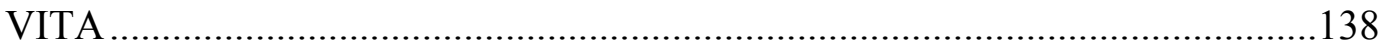




\section{LIST OF TABLES}

Table

Page

1. Strongest Program Evaluation Approaches ............................................23

2. Research Aligned to Industry-wide Indicators ....................................100 


\section{LIST OF FIGURES}

Figure Page

1. Program Evaluation Gradient ............................................................ 10

2. Review of Literature as Schema for Study ......................................... 18

3. UFE Approach Relative to the Other 25 Recognized Approaches............26

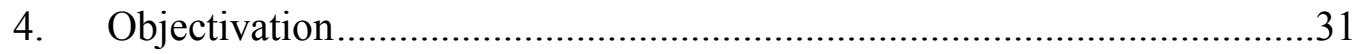

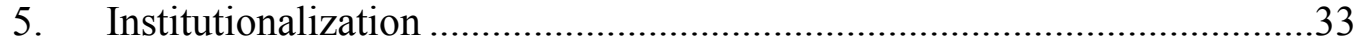

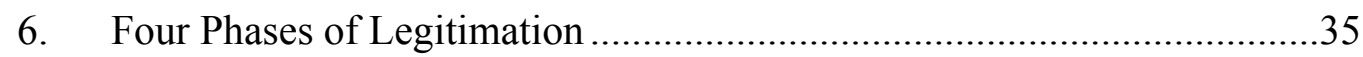

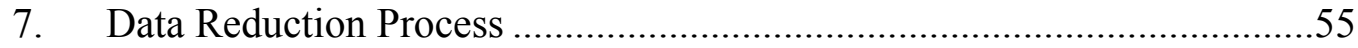

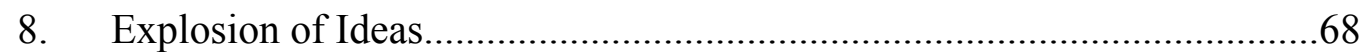

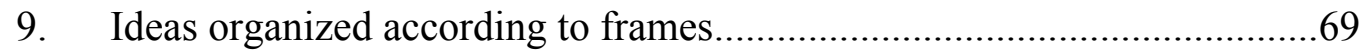

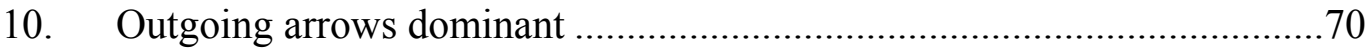

11. Procedural issues beget implications .............................................. 71

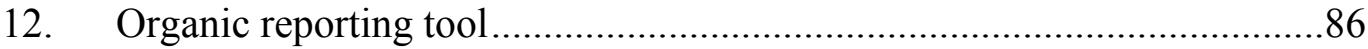

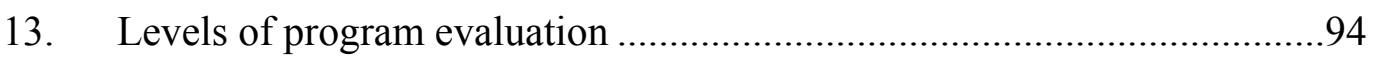




\title{
UTLIZATION-FOCUSED EVALUATION OF THE PROGRAM EVALUATION PROCESS OF A MISSOURI SCHOOL DISTRICT
}

\author{
Laura Nelson \\ Dr. Joyce Piveral and Dr. Phil Messner, Dissertation Supervisors
}

\begin{abstract}
The purpose of this study was to provide a utilization-focused evaluation (Patton, 1997) of a Missouri School District (hereafter referred to as The School District) program evaluation process. The distinguishing feature of the utilization-focused evaluation (UFE) approach is that it emphasizes intended use by clearly identified intended users. For this UFE, the primary intended user was the superintendent of a Kindergarten-12 (K-12) school district in Missouri; other stakeholders for this study were identified as the highest level of leadership within The School District. This group of stakeholders is collectively referred to as the superintendent's cabinet.

Literature suggests that the K-12 arena is not populated with professional program evaluators. As a result, evaluations typically emphasize outcomes at the expense of process assessment while also prioritizing compliance needs ahead of other stakeholder interests (Eddy \& Berry, 2007). The problem addressed by this study was that The School District had not yet determined how to implement program evaluations that are routinely capable of maximizing the use of stakeholder time, overcoming geographic constraints, and providing anonymity where necessary while still promoting organizational knowledge creation (Nonaka \& Takeuchi, 1995). The School District needed a program evaluation model that balanced organizational knowledge creation with cost effectiveness, efficiency, and compliance.
\end{abstract}


This study employed qualitative research processes and used the social construction of reality theory as a conceptual underpinning. The use of qualitative processes allowed for the collection of rich, thick descriptions (Merriam, 1998), which was particularly important given that the social construction of reality theory is predicated upon the importance of words, phrases, and narratives to construct meaning and diffuse knowledge (Berger \& Luckmann, 1967).

The chief research question asked whether or not a site-specific model for program evaluation could be generated. In response to the question, the study found that specific tools and processes could be generated. By implementing these tools, procedural issues could be rectified, which would, in turn, impact four facets of the organization: human relations, political, symbolic, and structural. However, the research also indicated a need for a fluidity in thought and practice that transcends the discrete processes normally conceived of within the confines of a model or what Morgan (1997) would call a "flexible style of organization and management" (p.44). Consequently, the study resulted in two types of conclusions-- procedural and process-use. Of particular interest is that the research findings could be aligned to three of the four evaluation industry-wide indicators of process use: enhancing shared understandings, increasing engagement, and program and organizational development (Pattton, 1997; King, 2007; Harner \& Preskill, 2007).

The most significant findings of the UFE were that a) The School District should implement clearly articulated processes, tools, and procedures, such as the type created as a result of this study, and b) The School District should also intentionally incorporate 
process-use within future evaluation practice as a method to deliberately provide for evaluation-capacity building and knowledge creation within the organization. 


\section{CHAPTER ONE \\ INTRODUCTION TO THE STUDY}

The No Child Left Behind Act (NCLB) of 2001 requires K-12 organizations to perform numerous program evaluations, despite the fact that few of these organizations have the professional capacity to support program evaluations beyond the minimum level required to achieve federal compliance (Rossi, Lipsey, \& Freeman, 2004; Scriven, 1996). Since the K-12 arena is not populated with professional program evaluators, evaluations typically emphasize outcomes at the expense of process assessment while also prioritizing compliance needs ahead of other stakeholder interests (Eddy \& Berry, 2007). As early as 2002, the Department of Education expressed concerns that "evaluation studies are not as helpful as they could be to practitioners at the local level, nor can they answer questions of causation" (New Directions, 2004). The selected Missouri school district, hereafter referred to as The School District, has not yet determined how to implement program evaluations that are routinely capable of maximizing the use of stakeholder time, overcoming geographic constraints, and providing anonymity where necessary while still promoting organizational knowledge creation.

Increasingly, leadership graduate programs are striving to provide their students with the tools to redress the imbalance of compliance and knowledge creation. At least one major university and its partners have intentionally trained their students on the practice of one form of collaborative evaluation: the utilization-focused approach, particularly as it is used within a paradigm designed to promote organizational development and knowledge creation. 
One leader trained by this program, the superintendent of The School District, created a department of assessment and program evaluation as a mechanism to comply with federal, state, and local mandates while at the same time engendering organizational development and knowledge creation. The superintendent, in conjunction with her cabinet, sought to address the challenge of reconciling what evaluation meant under NCLB with what it meant within the utilization-focused/organizational knowledge creation paradigm. In addition to the superintendent, the cabinet consisted of eight administrators who lead the district's chief areas of service: Curriculum, Instruction, and Assessment; Elementary Education; Secondary Education; Human Resources; Community Relations; Finance; Special Education; and Technology and Operations. The superintendent and her team recognized it is not cost effective to organize the district's program evaluation model, which heretofore had been non-existent, around a participatory evaluative model heavily predicated upon multiple stakeholders' meetings. Program stakeholders in the K-12 arena are comprised of district level personnel and building administrators. Stakeholders for this study believed only the highest priority program evaluations should rely upon convening high paid people to function as a think tank (personal communication, September 19, 2007).

Conceptual Underpinnings of the Study

Conceptual underpinnings are the principles that will be used to help explain cause and effect processes within the research and facilitate interpretation of research observations resulting from data analysis. This section articulates the research paradigm, the conceptual framework- social construction of reality theory- and the particular evaluative design, the utilization-focused approach. 


\section{Research Paradigm}

To best understand how processes and meanings evolve from any research, the reader is well-served to understand the cognitive logic of the researcher. Coghlan and Brannick (2005) emphasize that "researchers' epistemological and ontological perspectives legitimate their own distinctive way of doing research and determine what they consider as a valid, legitimate contribution to knowledge or theory" (p. 5).

Epistemology, the study of the theory of knowledge, concerns itself with questions regarding the essence and origin of knowledge. Again, the discipline is separated into objectivist and subjectivist leanings. Interpretation of language serves as a distinguishing factor. The researcher is an epistemological subjectivist, and, as such, "denies the possibility of a theory-neutral language" (Coghlan \& Brannick, 2005, p. 5).

This research is framed by a decidedly subjectivist ontological point of view which stipulates that individual perception is reality (Coghlan \& Brannick, 2005). As such, reality hinges on individual cognition; it has multiple frames of reference-- as opposed to an objectivist view that alternatively argues there is a single reality that hinges on external determinants. The confluence of these subjective stances creates an interpretivist/constructivist research stance.

\section{Social Construction of Reality Theory}

The conceptual framework for this research is Berger and Luckmann's (1967) social construction of reality theory, within which lies several tenets particularly pertinent to the aims of this study: objectivation, institutionalization, and legitimation. To more fully grasp the meaning of these ideas, it is useful to extrapolate the particular nuances of each as they relate to this study. 
Objectivation. Objectivation is the critical action Berger and Luckmann (1967) stipulate for creating "the foundations of knowledge in everyday life" (p. 19). It is a critical process for creating commonly held knowledge and is evidenced in the process of producing commonly understood evidence of action. Objectivation is what happens when people work together to produce a tangible product or reified concept that is mutually recognizable and understandable to not only the person or people whose activity produced it, but also to other individuals within the community. Integral to objectivation is the idea that individuals possess knowledge of everyday life in terms of structured relevances, which may or may not intersect with the relevance structures of other people (p. 45). Objectivation generates commonly understood evidence of action, and therefore knowledge, in large part because common action realigns individual relevance structures. Institutionalization. A second process within the social construction of reality theory (Berger \& Luckmann, 1967) is institutionalization. It "occurs whenever there is a reciprocal typification of habitualized actions by types of actors" (Berger \& Luckmann, 1967, p. 54). In other words, institutionalization is what happens when the habitualized actions and/or modes thinking of an individual are transferred in such a way that these same actions and/or modes of thinking become the habitualized behavior of the group. "Men together produce a human environment" ... and "social order is a human product, or, more precisely, an ongoing human production" (Berger \& Luckmann, p. 51). Berger and Luckmann go on to explain, "The objectivated meanings of institutional activity are conceived of as 'knowledge' and transmitted as such. Some of this 'knowledge is deemed relevant to all, some only to certain types. All transmission requires some sort of apparatus" (p. 70). 
Legitimation. Legitimation, the third and final "social construction of reality" concept, "produces new meanings that serve to integrate the meanings already attached to disparate institutional processes" (Berger \& Luckmann, 1967, p. 92). It takes place in four phases and is important because it results, according to Berger and Luckmann, in the "full integration of discrete institutional processes" (p. 96). Legitimation is grounded in discourse and is built upon objectification and institutionalization. This concept is important to the study because it raises the expectation that the resulting model would function as a mechanism capable of integrating discrete philosophies and processes and of uniting them under the umbrella of the three School District values: culture of collaboration, integrity of action, and commitment to excellence.

Utilization-Focused Evaluation. The evaluation lens for this study was the Utilization-Focused Evaluation (UFE) approach. When contrasting the UFE approach to program evaluation in general, the key distinction rests in the premise that "utilizationfocused program evaluation is...done for and with specific, intended primary users for specific, intended uses" (Patton, 1997, p. 23). The UFE approach is "explicitly geared to ensure that program evaluation makes an impact" (Stufflebeam \& Shinkfield, 2007, p. 230). Patton notes that this approach begins with identifying stakeholders and working with them to understand first, what it is they want to know as a result of the evaluation, and second, how they intend to use the results.

Summary of conceptual underpinnings. This research was underpinned by the social construction of reality (Berger \& Luckmann, 1967) premises. Further, it was conducted within the scope of the Utilization-Focused Evaluation approach, which is inherently qualitative in nature. The qualitative design is "especially appropriate for 
developing, innovating, or changing programs in which the focus is improving the program, facilitating more effective implementation, and exploring a variety of effects on participants" (Patton, 1997, p. 286).

The confluence of these ideas seems to mean two things for organizational knowledge creation. First, the structure of the study itself has the potential to engender learning among the stakeholders. Second, the findings of the study have the potential to be subject to objectivation, institutionalization, and legitimation.

Statement of the Problem

The School District has not yet determined how to implement program evaluations that are routinely capable of maximizing the use of stakeholder time, overcoming geographic constraints, and providing anonymity where necessary, while still promoting organizational knowledge creation. The School District needs a program evaluation model that balances organizational knowledge creation with cost effectiveness, efficiency, and compliance.

\section{Purpose of the Study}

The purpose of this study is to conduct a Utilization-Focused Evaluation (Patton, 1997) of The School District's program evaluation process. The study will generate for The School District a site-specific process and model for program evaluations in the future.

\section{Research Questions}

To address the purpose of the study, the following research questions will guide the study: 
1. Can a site-specific model for program evaluation be generated?

2. What data can be gathered to inform the creation of the program evaluation model?

3. How will data be analyzed to inform the creation of the model?

\section{Methodology}

The superintendent of The School District requested a Utilization-Focused Evaluation (Patton, 1997) of the district's program evaluation process. The superintendent and her council desired a cost-effective, program evaluation model that was flexible enough to provide information, speak to programming causation, ensure state and federal compliance, while also promoting organizational knowledge creation. The researcher utilized multiple data sources, including federal, state, and local documents; archival data; current data; an electronic survey; and in-depth, one-on-one interviews in order to satisfy the identified purpose of the evaluation.

Data analysis took place on multiple levels. Content analysis was performed upon four discrete sets of documents: The No Child Left Behind Act of 2001 (NCLB); Missouri School Improvement Program (MSIP)- Cycle 4; The School District's board policy; and The School District's vision, mission, and goals. Once content analysis was complete, an affinity diagram was created. Brassard (1996) notes, "this tool gathers large amounts of language data, organizes it into groupings based on the natural relationships between each item, and defines groups of items. It is a largely creative process" (p. 17). Content analysis of archival data from The School District's program evaluation pilot was performed, and then it was analyzed utilizing an interrelationship diagraph. Again, Brassard explains, "this tool takes a central idea, issue, or problem and maps out the 
logical or sequential links among related items." Though it is very much like an affinity diagram, it is not the same in that "an affinity allows some initial organized creative patterns to emerge but an interrelationship diagraph lets logical patterns become apparent" (p. 41). Open and axial coding was utilized to reduce data gathered from surveys and interviews. These processes allowed the researcher to first "fracture the data...to identify some categories, their properties, and dimensional locations" and then put the data "back together in new ways by making connections between a category and its subcategories" (Heppner \& Heppner, 2004, p. 152). In constructing the electronic survey, the Delphi technique was utilized to integrate data and ensure all stakeholder ideas are properly represented. Garavalia and Gredler (2004) note that this "technique permits the collection of rich evaluation data" (p. 375). The member check strategy was used throughout the process in order to provide for internal validity. Data collection and analysis followed a particular sequence.

1. Interview the primary intended user- the superintendent- via the first long interview and analyze the transcript.

2. Analyze documents: No Child Left Behind Act of 2001, Missouri School Improvement Program (MSIP 4), and The School District's board policy.

3. Analyze archival data from The School District's program evaluation pilot.

4. Interview stakeholders-superintendent's cabinet- via individual interviews and analyze transcripts.

5. Utilize Survey Monkey (2008) for electronic survey of stakeholders.

6. Interview the primary intended user- the superintendent- via second long interview and analyze transcript. 


\section{Significance of the Study}

This evaluation was designed to provide a program evaluation model for The School District. The model provided the district with a replicable process for use on future program evaluations and included several non-exclusive outcomes: compliance with federal, state, and local mandates, process analysis, and organizational knowledge creation. Since the model is potentially transportable, this evaluation may benefit other organizations within the K-12 arena, in so far as other districts may be able to transpose the model upon their unique settings and glean the aforementioned non-exclusive benefits.

The program evaluation gradient can be conceptualized as a line that contains at its left extreme a compliance-based method for program evaluation, which consumes few organizational resources. On the other extreme rests the fully inclusive UtilizationFocused Evaluation, which consumes many organizational resources, but arguably also carries greater potential for organizational knowledge creation. The vector, which has direction as well as magnitude, allows one to determine where a particular type of evaluation model fits relative to another type. 


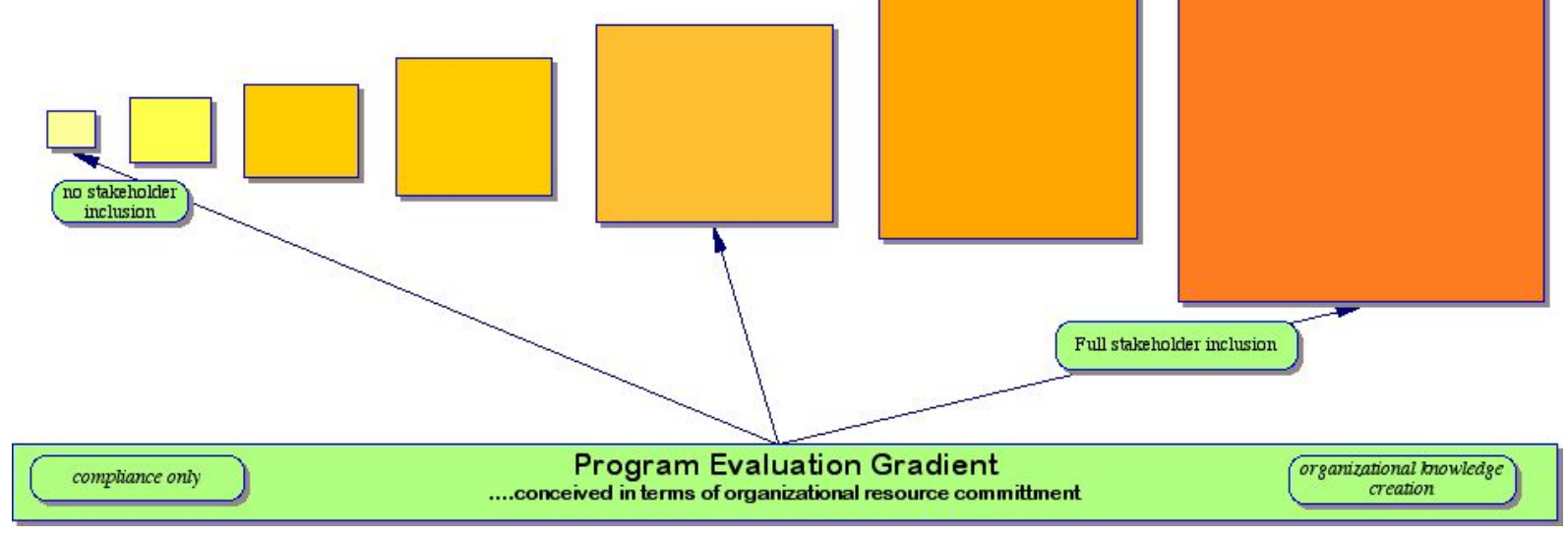

Figure 1. The School District's program evaluation model is projected to be situated in the middle of the gradient.

\section{Definition of Terms}

This section will provide definitions of key conceptual and operational constructs as they are applicable within the confines of this study. These terms are particularly relevant to the qualitative nature of the research.

Affinity diagram. Brassard (1996) explains, "this tool gathers large amounts of language data, organizes it into groupings based on the natural relationships between each item, and defines groups of items. It is a largely creative... process" (p.17).

Axial coding. This is the process of putting data derived from the open coding process together in new ways by making connections between categories (Heppner \& Heppner, 2004).

Coding. This is a data analysis strategy known as the first step in taking an analytic stance toward the data. It gives the research analytic scaffolding on which to build. The two chief forms of coding are open and axial (Denzin \& Lincoln, 2005). 
Compliance. When the term is used in this study it refers to the act of undertaking an evaluation for the sole purpose of complying with a legislative mandate.

Evaluation approach. This term refers to a broad conceptualization of evaluation methodologies and practices; it encompasses evaluation models. There are 26 routinely recognized evaluation approaches. See Appendix A for complete list (Stufflebeam \& Shinkfield, 2007).

Institutionalization. Institutionalization is what happens when the habitualized actions and/or modes thinking of an individual are transferred in such a way that these same actions and/or modes of thinking become the habitualized behavior of the group. One of the three key processes of the social construction of reality, institualization is founded upon objectification. In conjunction with objectification, it provides the foundation for legitimation (Berger \& Luckmann, 1967).

Interrelationship diagraph. Brassard (1996) explains "this tool takes a central idea, issue, or problem and maps out the logical or sequential links among related items...[it] lets logical patterns become apparent” (p. 41).

Legitimation. Legitimation produces new meanings that serve to integrate the meanings already attached to disparate institutional processes. It is founded upon objectification and institutionalization and is one of three key processes in the social construction of reality.

No Child Left Behind Act of 2001. This federal legislation demands systematic data on the performance of public education. One section of the legislation particularly mandates an emphasis upon on-going program evaluation. 
Objectivation. This is the process of producing commonly understood evidence action. To be considered an objectivated product, it must be mutually understandable by both the product's creator(s) and its audience(s). It is the first key process in the social construction of reality (Berger \& Luckmann, 1967).

Open coding. This process involves the examination of minute sections of text made up of individual words, phrases, and sentences and is one of the chief data analysis strategies of this study (Heppner \& Heppner, 2004).

Organizational knowledge creation paradigm. According to Smith (2007) such a paradigm is established by a leader who promotes life-long learning, sets high expectations, makes data-driven decisions, and aligns resources with learning (p. 34).

Primary intended user. The stakeholder identified as the individual who "personally care[s] about the evaluation and the findings it generates" (Patton, 1997, p.44) and, for the purposes of this study, The School District's superintendent.

Program evaluation. According to Wholey, Hatry, and Newcomer (2004) "Program evaluation is the systematic assessment of program results and, to the extent feasible, systemic assessment of the extent to which the program caused those results" ( $p$. xxxiii). As such, program evaluation is predicated upon a cause and effect model.

Program. A program can be thought of as the composite aggregation of resources and other inputs, program activities, outputs, and outcomes, all tied together by causality and framed by results achieved by the program's target population.

Relevance. Relevance is delegated differently by different people according to each person's values and priorities. It is used in this study to understand how individual 
relevance structures can potentially contribute and/or detract to the creation of organizational knowledge.

Relevance structures. These are probably best understood as mental models for the way an individual arranges his/her own relevance. Berger and Luckmann (1967) argue that one's relevance structures are constantly intersecting with those of other people.

Stakeholders. Those who are intended to use the findings, others who may be affected by the evaluation, and those expected to contribute to the study. These persons help affirm foundational values, define evaluation questions, clarify evaluative criteria, contribute needed information, help interpret findings, and assess evaluation reports (Stufflebeam \& Shinkfield, 2007). In addition to the primary intended user, the stakeholders for this study consist of the eight administrators who lead the district's chief areas of service: Curriculum, Instruction, and Assessment; Elementary Education; Secondary Education; Human Resources; Community Relations; Finance; Special Education; and Technology and Operations. Collectively, this group is known as the Superintendent's Cabinet.

Transportable. A program evaluation model is considered transportable when it can be conveyed from one location to another, such as from one evaluation to another or from one district to another.

Utilization-Focused Evaluation. Utilization-Focused Evaluation (UFE) is one of 26 approaches to evaluation recognized by Stufflebeam and Shinkfield (2007). Patton (1997) explains "utilization-focused program evaluation (as opposed to program 
evaluation in general) is evaluation done for and with specific, intended primary users for specific, intended use" (p. 23).

\section{Delimitations}

The following delimitations were identified for this study.

1. The study was confined to one school district. The School District was selected based upon its desire to implement a program evaluation model situated along a gradient of possibilities (Figure 1).

2. The study was confined to a district planning cycle and integration of document analysis and archival data.

\section{Limitations}

This study had several limitations:

1. The study was limited because The School District is only in the first phase of professional program evaluation.

2. The study was limited because it was based in large part upon the respondents' perceptions.

3. The study was limited because the researcher is the research instrument.

4. The study was limited because it is subject to interpretation and analysis by the researcher who is employed by The School District.

\section{Summary}

The School District has not yet determined how to implement program evaluations that are routinely capable of maximizing the use of stakeholder time, overcoming geographic constraints, and providing anonymity where necessary while still 
promoting organizational knowledge creation. The School District needs a program evaluation model that balances organizational knowledge creation with cost effectiveness, efficiency, and compliance issues. The purpose of this study was to conduct a Utilization-Focused Evaluation (Patton, 1997) of the program evaluation process of The School District. The study provided The School District a site-specific process and model for future program evaluations. 


\section{CHAPTER TWO}

\section{REVIEW OF LITERATURE}

Introduction

Problem, Purpose, Significance, and Delimitations of the Study

Problem. The School District has not yet determined how to implement program evaluations that are routinely capable of maximizing the use of stakeholder time, overcoming geographic constraints, and providing anonymity where necessary while still promoting organizational knowledge creation. The School District needs a program evaluation model that balances organizational knowledge creation with cost effectiveness, efficiency, and compliance.

Purpose. The purpose of this study was to provide a Utilization-Focused Evaluation (Patton, 1997) of The School District's program evaluation process. The study provided The School District a site-specific process and model for program evaluations in the future.

Significance. This evaluation was designed to provide a program evaluation model for The School District. The model provided the district with a transportable process, one which can be used on future program evaluations to support several nonexclusive outcomes: compliance with federal, state, and local mandates, process analysis, and organizational knowledge creation. This evaluation might also be of benefit to other organizations within the K-12 arena.

Delimitations of the Study. There were several parameters on the study. The study was confined to one school district. The School District was selected based upon its 
desire to implement a program evaluation model situated along a gradient of possibilities. The study was confined to a district planning cycle.

Review of Literature as Schema for the Study. A review of related literature for this study will provide a schema for the proposed research, while at the same time allowing the reader the opportunity to examine the anticipated integration of ideas. A reification of the schema provides a useful roadmap for the review of literature (Smith, 2007). 


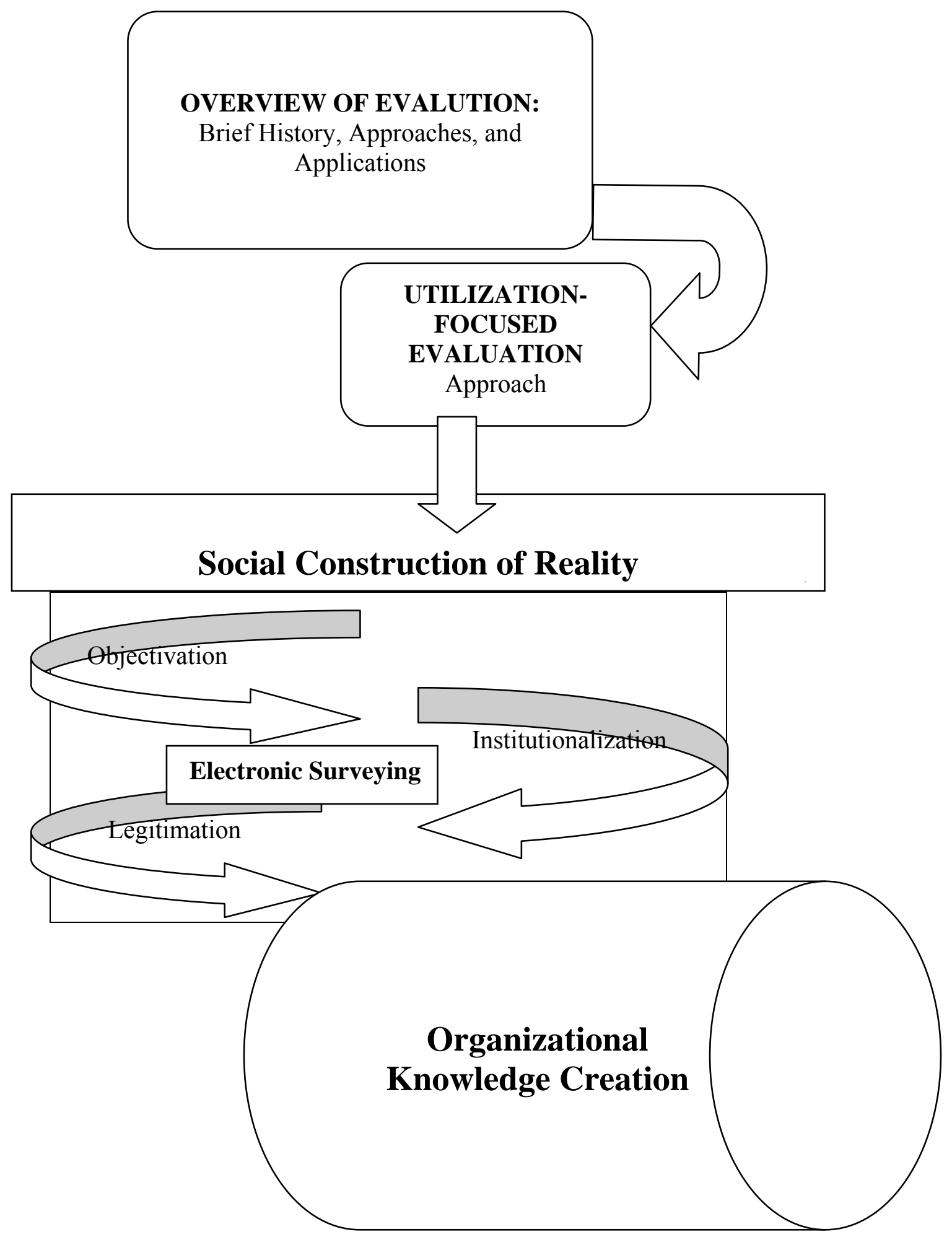

Figure 2. The review of literature serves as a schema for the study. 
A broad contextual background regarding evaluation provides an important starting point. To clarify the role of UFE relative to the rest of the art, science, and profession of evaluation, it is necessary to begin with a brief overview of evaluation history, approaches, and applications. From there, the review will turn to the UFE approach, with particular emphasis on aspects most likely to engender the social construction of reality, the chief conceptual underpinning of the study. It will address Bergman and Luckmann's (1967) three important tenets: objectification, institionalization, legitimation. It will also support the use of electronic surveying. Finally, the review of literature will elaborate upon how these ideas might be expected to impact organizational knowledge creation.

Overview of Evaluation History, Approaches, and Applications. To clarify the role of UFE relative to the rest of the art, science, and profession of evaluation, it is necessary to begin with a brief overview of evaluation history, approaches, and applications. These ideas are included to provide schema regarding the evolution of evaluation as it relates to this research.

Evaluation history. The evaluation of social programming can be traced back to the beginning of the $20^{\text {th }}$ century, where it is known to have originated, in large part, in the field of education (Rossi, Lipsey \& Freeman, 2004; Scriven, 1996). According to Freeman (1977), "by the 1930's, social scientists were using rigorous research methods to assess social programs in a variety of areas" (p. 17). By the "end of the 50's program evaluation was common place." It was a "growth industry" by the 1960's and "emerged as a distinct specialty field" by the 1970's (Rossi, Lipsey, \& Freeman, 2004, p. 9). In 1994, the American Evaluation Association developed and endorsed principles to guide 
the profession: systemic inquiry, competence, integrity/honesty, respect for people, and responsibilities for general and public welfare. The committee, commonly called The Joint Committee, also developed The Standards for Program Evaluation: utility, feasibility, propriety, and accuracy (Patton, 1997). To this day, scholars concur that The Standards are the benchmark against which all evaluations must be measured (Patton; Preskill \& Torres, 1998; Rodriguez-Campos, 2005; Rossi, Lipsey, \& Freeeman; Stufflebeam \& Shinkfield, 2007). The AEA is now 20 years old and has more than 5,200 members who represent 60 countries. As society advances, it is important to note that the "evaluation enterprise [is] powerfully shaped by changing times" (Rossi, Lipsey, \& Freeman, 2004, p. 15). Over the course of evaluation's history, one issue has "persistently plagued evaluation researchers and practitioners... the use of evaluation findings" (Preskill \& Donaldson, 2008, p. 113). It was out of concern for persistent and pervasive lack of use of evaluation findings that Patton (1997) and others began to seriously investigate and implement the utilization-focused design for evaluations.

Evaluation approaches. Evaluation in practice tends to look very much like traditional academic research. Both are driven by research questions. Both must identify data collection and analysis methods. Both typically generate meaning and provide recommendations for future actions. As in traditional academia, the evaluation field of practice and practitioners spans the rich spectrum of quantitative and qualitative methodologies (Patton, 1997; Preskill \& Torres, 1998; Rodriguez-Campos, 2005; Rossi, Lipsey, \& Freeeman; Stufflebeam \& Shinkfield, 2007) However, there is a distinction to be made: "a distinguishing characteristic of evaluation is that unlike traditional forms of academic research, it is grounded in the everyday realities of organizations" (Russ-Eft \& 
Preskill, 2001, p. 6). Patton (1997) categorizes evaluation approaches according to focus. In this way, he negotiates the distinction between approaches based upon the types of questions a given approach is best suited to answering. He provides 58 possible options, but notes that his list "by no means exhaust[s] all possibilities...and encourages evaluators to remember various options can be and often are used to together within the same evaluation or in a sequence over time" (pp. 192-194). Newcomer, Hatry, and Wholey (2004) are proponents of just such a sequential approach to program evaluation. Indeed, several of the types found on Patton's (1997) list are specifically noted key elements in what they deem "site-specific" program evaluation: logic modeling, evaluability assessment, implementation evaluation, ongoing performance monitoring, and experiential (or quasi) designs (p. 1).

Stufflebeam and Shinkfield (2007) distill the approaches to a more concise list of 26 (Appendix A). While this list possesses echoes of Patton's (1997), for the most part, it subsumes the details found in Patton's work and groups the 26 identified approaches into five categories (See table 1). The categories are listed here to provide schema regarding an overview of the field of evaluation approaches and to identify the location of the UFE approach relative to other generally accepted approaches.

1. Pseduoevaluations (5 approaches)

2. Questions-and Methods-Oriented Evaluations/Quasi-Evaluation Studies (14 approaches/ 3 in top 8)

3. Improvement-and Accountability-Oriented Evaluation (3 approaches/ 2 in top 8)

4. Social Agenda and Advocacy Evaluation (3 approaches/ 2 in top 8) 


\section{Eclectic Evaluation (1 approach (UFE)/ 1 in top 8)}

Additionally, Stufflebeam and Shinkfield argue that among the 26 approaches, eight stand out in terms of their ability to function in terms of the standards put forth by The

Joint Committee: utility, feasibility, propriety, and accuracy. These eight approaches can be rank-ordered according to overall score and rating. Of significance to this study is the placement the UFE approach, as well as the individual ratings of each evaluation standard. Because the UFE was the instrument used during this research, projections regarding the utility, feasibility, propriety, and accuracy may be generalizable to this study. 
Table 1

Strongest Program Evaluation Approaches, According to Stufflebeam and Shinkfield (2007), as Measured by Compliance with The Program Evaluation Standards

\begin{tabular}{llllll}
\hline $\begin{array}{l}\text { Evaluation } \\
\text { Approach }\end{array}$ & $\begin{array}{l}\text { Overall } \\
\text { Score } \\
\text { and } \\
\text { Rating }\end{array}$ & $\begin{array}{l}\text { Utility } \\
\text { Rating }\end{array}$ & $\begin{array}{l}\text { Feasibility } \\
\text { Rating }\end{array}$ & $\begin{array}{l}\text { Propriety } \\
\text { Rating }\end{array}$ & $\begin{array}{l}\text { Accuracy } \\
\text { Rating }\end{array}$ \\
\hline CIPP Model & 92 & 93 & 92 & 88 & 94 \\
$\begin{array}{l}\text { Utilization- } \\
\text { focused }\end{array}$ & 86 & 93 & 92 & 78 & 79 \\
$\begin{array}{l}\text { Consumer } \\
\text { oriented }\end{array}$ & 84 & 89 & 75 & 91 & 81 \\
$\begin{array}{l}\text { Responsive and } \\
\text { client centered } \\
\text { Case study }\end{array}$ & 84 & 93 & 83 & 78 & 81 \\
$\begin{array}{l}\text { Constructivist } \\
\text { Objectives }\end{array}$ & 81 & 81 & 83 & 81 & 88 \\
$\begin{array}{l}\text { based } \\
\text { Experimental }\end{array}$ & 56 & 61 & 67 & 84 & 85 \\
$\begin{array}{l}\text { design } \\
\text { Note: Stufflebean }\end{array}$ & 54 & 42 & 53 & 73 \\
\hline
\end{tabular}

Note: Stufflebeam and Shinkfield, 2007, p. 242- as modified by the researcher

Evaluation applications. The demand for program evaluation is growing;

consequently, so are the potential applications. Government offices at all levels, federal, state, and local, are increasingly calling for the auditing of public programs. Such auditing typically revolves around compliance issues and the need for data on "economy, efficiency, and return on investment." (Newcomer, Hatry \& Wholey, 2004, p. xxxviixxxviii). An array of legislation mandates program evaluation; the applications most relevant to this study stem from The No Child Left Behind Act of 2001 (NCLB) and Missouri School Improvement Program (MSIP), cycle 4. Stakeholder commitment to compliance and auditing-oriented appraisals emerged within the findings of this study; 
however, it is important to remember that this study was commissioned based on the need to go beyond this particular application.

Beyond the auditing function, Patton (1997) details a variety of contrasting applications: "internal versus external; outcomes versus process; experimental designs versus cases studies; mandated accountability systems versus voluntary management efforts; academic studies versus information action research by program staff” (p. 64). Stufflebeam and Shinkfield (2007) argue evaluation is "society's most fundamental discipline...oriented to assessing and improving all aspects of society" (p.4). Increasingly, evaluation is used to facilitate learning-- "especially transformative learning in organizational contexts" (Preskill \& Torres, 2000, p.25).

Utilization-Focused Evaluation (UFE) When contrasting the UFE approach to program evaluation in general, the key distinction rests in the premise "utilizationfocused program evaluation is...done for and with specific, intended primary users for specific, intended uses" (Patton, 1997, p. 23). When thinking about this, it is helpful to realize that prior to the 1970s, evaluation was largely approached via experimental design, an approach that, to this day, is applied. However, evaluations done outside the organizational context are often perceived by intended users as something being done to, or on, them. As a result, evaluation findings are often relegated to a forgotten shelf, where they have little chance of impacting organizational development and/or knowledge creation (Patton, Preskill \& Torres, 1999; Stufflebeam \& Shinkfield, 2007). The Utilization-Focused Evaluation (UFE) approach is "explicitly geared to ensure that program evaluation makes an impact” (Stufflebeam \& Shinkfield, 2007, p. 230). Moreover, of the 26 recognized approaches to evaluation, Stufflebeam and Shinkfield 
deem the UFE approach "eclectic," noting "all [other] evaluation methods are fair game in a Utilization-Focused Evaluation” (p. 232). 


\section{UFE Approach Relative* to the Other 25 Recognized Evalaution Approaches}
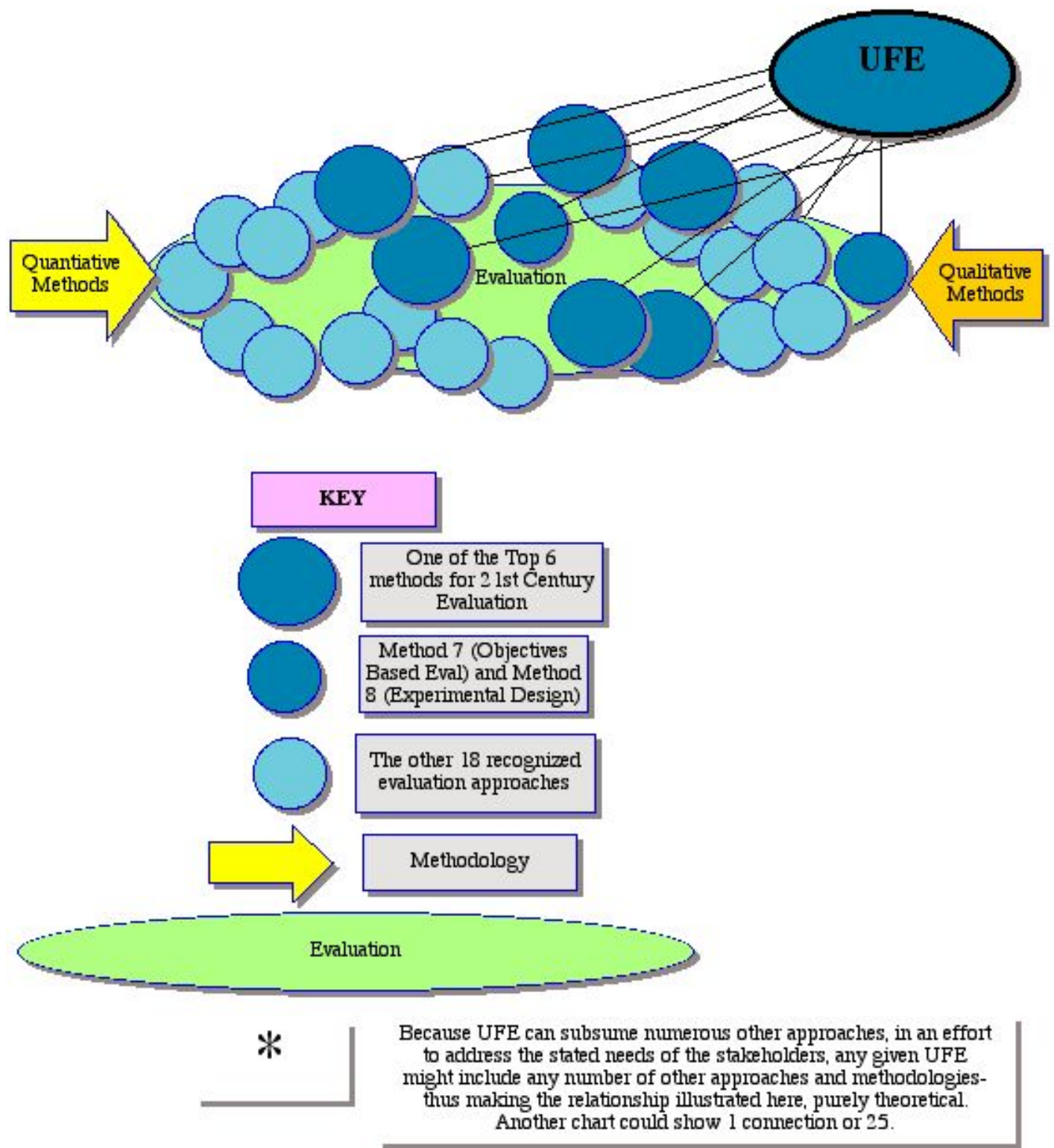

Figure 3. The Utilization-Focused Evaluation (UFE) approach often draws on other evaluation approaches (Patton, 1997; Stufflebeam \& Shinkfield, 2007). 
Stufflebeam and Shinkfield (2007) rank the Utilization-Focused Evaluation as the second strongest evaluation approach as weighted in terms of compliance with the widely recognized Program Evaluation Standards: utility, feasibility, propriety, accuracy (AEA, 1995). As such, they argue it is one of the six "best approaches for $21^{\text {st }}$ Century Evaluations" (p. 244). Horton (1999) argues that this approach provides the evaluator with a "broad theoretical framework that puts the various evaluation theories, approaches, and controversies in perspective, as parts of a broader whole," while at the same time providing "methodological options and site-specific guidelines that are consistent with the theory and have proven useful in practice" (p. 399).

Though widely attributed to Patton, the Utilization-Focused Evaluation (UFE) approach has been around in utilization-theory circles since early in the 1970's (Alkin, 2004). Indeed, "some evaluation theorists realized early that one vital aspect of the success of any evaluation is the extent to which it can bring about discernable change and how this can be achieved" (Stufflebeam \& Shinkfield, 2007, p. 431). However, with the 1978 publication of the first edition of Utilization-Focused Evaluation, Patton became widely recognized as the father of the form. Publication numbers stand as testament: the third edition, published in 1997, necessitated five printings; Patton is currently preparing the $4^{\text {th }}$ edition for press.

The UFE approach (Patton, 1997) begins with identifying stakeholders and working with them to understand first, what it is they want to know as a result of the evaluation, and second, how they intend to use the results. Analogues for this method can be found in Preskill and Torres' (1998) evaluative inquiry approach, as well as Rodriguez-Campos' (2005) version of collaborative evaluation. Patton argues that 
beginning an evaluation this way serves to "engender commitments to both evaluation and use" (p. 21). Emphasis on the collaborative relationship between and among the evaluator and stakeholders empowers stakeholders to interact and learn from one another. As a result, Lencioni's (2002) premise that a team can and should work to integrate members' values, needs, and beliefs is addressed. Patton deems such integrative benefits "process use" of the evaluation and explains "being engaged in the evaluation can be useful quite apart from the findings that may emerge from the process" (p. 88).

Once the stakeholders are identified and the groundwork for the evaluation is set, Patton (1997) argues it is necessary for the evaluator to facilitate the focusing of the evaluation. He cites numerous ways to go about this, and specifically elaborates on four mechanisms: a) focus on future decisions; b) focus on critical issues or concerns; c) focus on responsive approach; and d) focus on questions (pp. 184-185). The importance of working with stakeholders to focus the evaluation merits further emphasis. Patton explains:

Focusing an evaluation means dealing with several basic concerns.

What is the purpose of the evaluation? How will the information be used? What will we know after the evaluation that we don't know now? What actions will we be able to take based on evaluation findings? ...As evaluators and primary users interact around these questions, the evaluation takes shape (pp.189190).

In a UFE design, stakeholder involvement is paramount. Once focused, appropriate methods must be vetted and mutually agreed upon. When the findings are aggregated, the evaluator must then facilitate stakeholders in the analysis and 
interpretation of the results. Stufflebeam and Shinkfield (2007) explain in this approach, "the evaluator...is the intended user's technical assistant...[he/she] must be an effective negotiator...working hard to gear a defensible program evaluation to the targeted users' evolving needs" (p. 232).

While the UFE approach clearly necessitates a considerable allocation of organizational time and resources (Patton, 1997), it is well-suited to engendering collaboration and what the leadership scholarship deems a key principle of change: people are more likely to embrace and value innovations (or this case evaluation use) if they were involved along the way (Bolman \& Deal, 2003; Fullan, 2001; Kotter, 1996; Lencioni, 2002; Starratt, 2003; Yukl, 2006).

Social Construction of Reality The collaborative nature of the UFE approach engenders the social construction of knowledge as framed within Berger and Luckmann's (1967) social construction of reality theory. Fundamental to this theory are two premises: a) the foundation of knowledge in everyday life is derived and maintained by social interaction and b) "the world originates in ...thoughts and actions and is maintained as real by these" (p. 20).

There are several tenets of Berger and Luckmann's theory which are particularly pertinent to the aims of this study: objectivation, institutionalization, and legitimation. To more fully grasp the meaning of these ideas, it is useful to consider the particular nuances of each as they relate to this study.

Objectivation. Objectivation is the critical action Berger and Luckmann (1967) stipulate for creating "the foundations of knowledge in everyday life" (p. 19). It is a critical process for creating commonly held knowledge and is evidenced in the process of 
producing commonly understood evidence of action. Objectivation is what happens when people work together to produce a tangible product or reified concept that is mutually recognizable and understandable to not only the person or people whose activity produced it, but also to other individuals within the community. Integral to objectivation is the idea that individuals possess knowledge of everyday life in terms of structured relevances, which may or may not intersect with the relevance structures of other people (p. 45). Objectivation generates commonly understood evidence of action, and therefore knowledge, in large part because common action realigns individual relevance structures.

The diagram illustrates objectivation and serves to provide a frame of reference for this study. The School District had a knowledge void pertaining to program evaluation-- the grey box. Moreover, a cabinet made up of individuals with highly divergent, unevenly spaced relevance structures regarding program evaluation was leading The School District. Individuals (and their relevance structures) are represented by the scattered pentagons. The UFE was the activity. The resulting product, the program evaluation model, was buttressed by the realigned relevance structures of the participants, whose input bolstered the model to varying degrees as represented by the placement of the pentagons. The study found that realignment of relevance structures is an important, desired process-use of program evaluation in The School District. 


\section{OBJECTIVATION:}

a critical process for producing commonly held knowledge
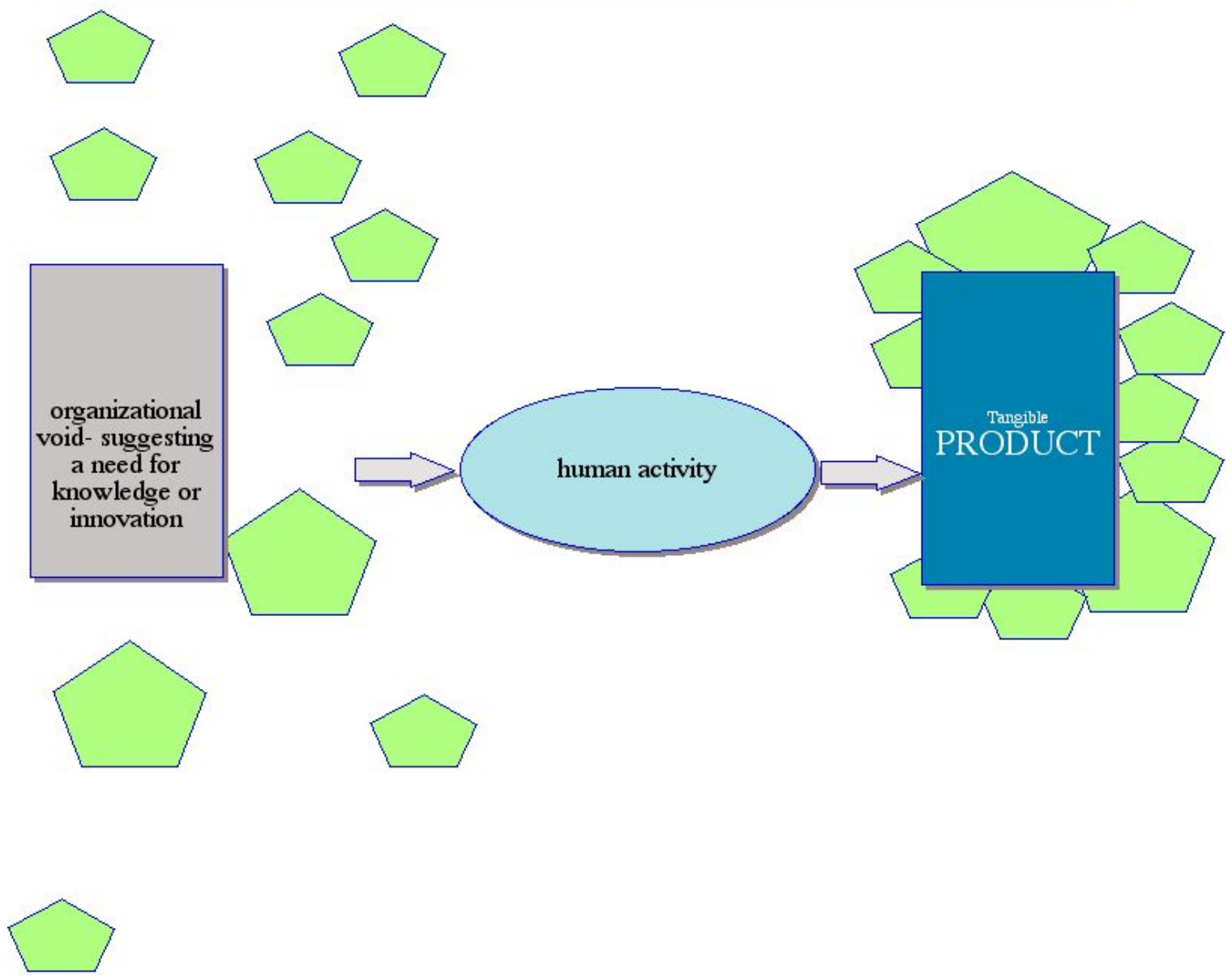

KEY

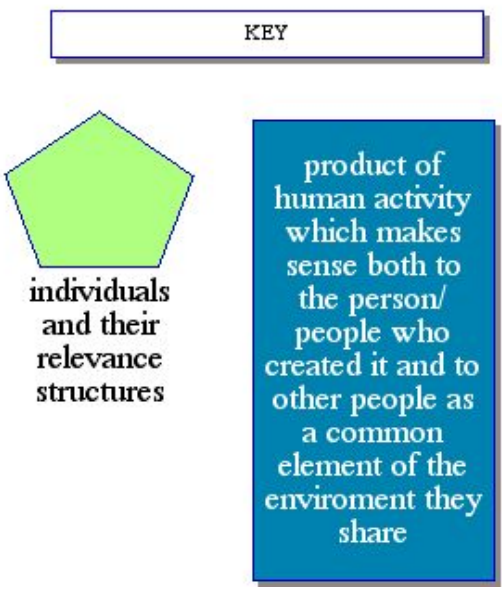

Figure 4. Objectivation is a process that aligns individual relevance structures. 
Institutionalization. A second process within the social construction of reality theory is known as institutionalization. It "occurs whenever there is a reciprocal typification of habitualized actions by types of actors" (Berger \& Luckmann, 1967, p. 54). In other words, institutionalization is what happens when the habitualized actions and/or modes thinking of an individual are transferred in such a way that these same actions and/or modes of thinking become the habitualized behavior of the group. "Men together produce a human environment" ... and " social order is a human product, or, more precisely, an ongoing human production" (Berger \& Luckmann, p. 51). Berger and Luckmann go on to explain "The objectivated meanings of institutional activity are conceived of as 'knowledge' and transmitted as such. Some of this 'knowledge is deemed relevant to all, some only to certain types. All transmission requires some sort of apparatus" (p. 70).

Again, the illustration serves to help link the concept to the study. The model and processes that are derived from this study have the potential to be transferred into the fabric of the organizational culture in the form of commonly held understandings and expectations of the stakeholders who have the potential to carry forth from the study commonly held expectations regarding how program evaluation should be done and what benefits stand to be gained in their district in the future. 


\section{Institutionalization:}

a critical process for producing commonly held knowledge

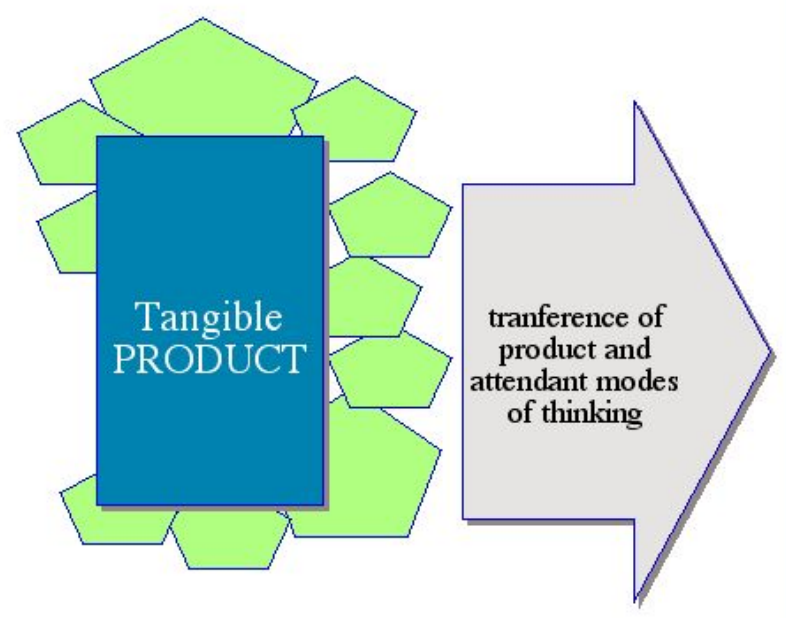

\section{Organizational Culture}

KEY
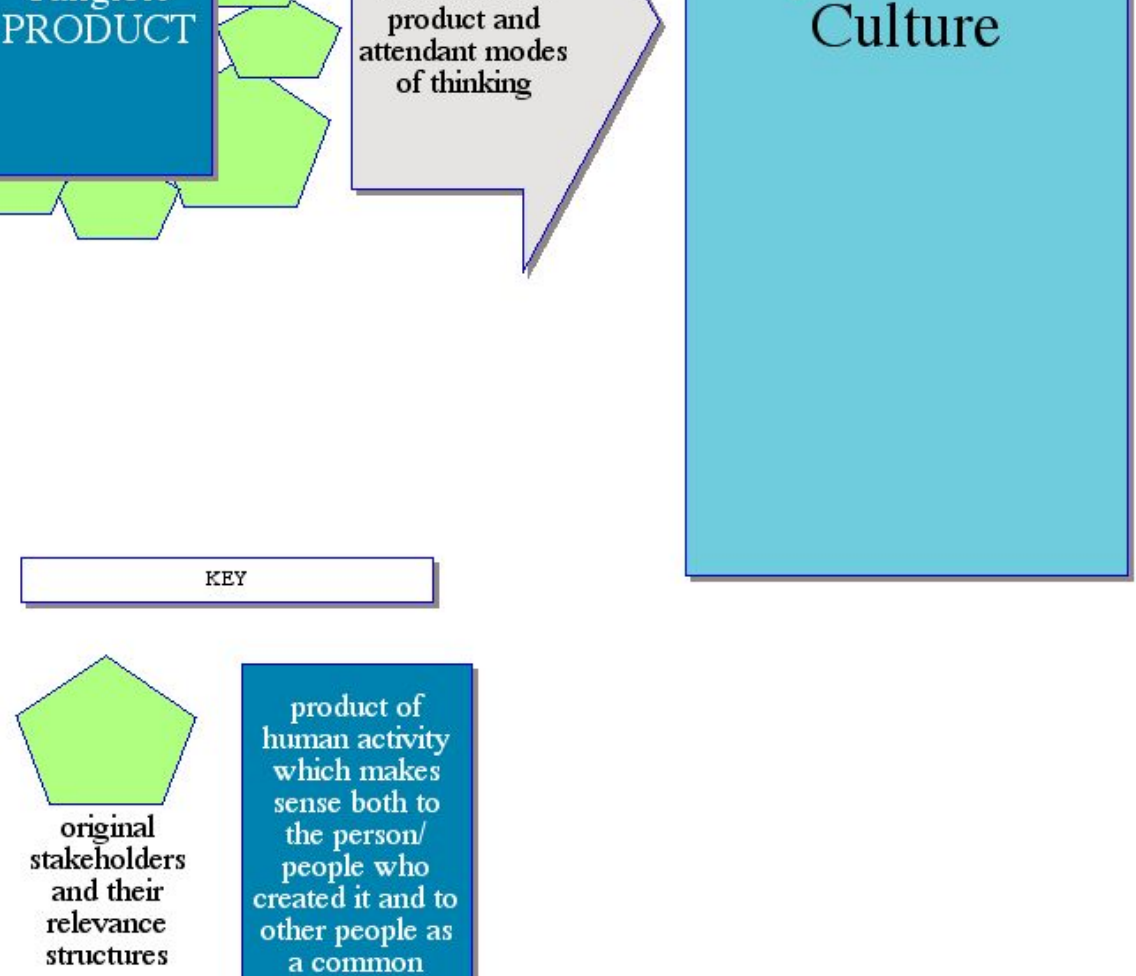

product of

human activity

which makes

sense both to

the person/

people who

created it and to

other people as

a common

element of the

environment

they share

Figure 5. Institutionalization transfers modes of thinking into organizational culture. 
Legitimation. Legitimation, the third and final social construction of reality concept, produces new meanings that serve to integrate the meanings already attached to disparate institutional processes" (Berger \& Luckmann, 1967, p. 92). It takes place in four phases and is important because it results, according to Berger and Luckmann, in the "full integration of discrete institutional processes" (p. 96). Legitimation is grounded in discourse and is built upon objectification and institutionalization. This concept is important to the study because it raises the expectation that the resulting model could function as a mechanism capable of integrating discrete philosophies and processes and of uniting them under the umbrella of the three School District values: culture of collaboration, integrity of action, and commitment to excellence.

There are four phases of legitimation. It is useful to think of these phases in terms of sequential stair steps leading to the goal of a fully integrated organization. Full integration is defined in this context as an organization whose processes work together to ensure district values are reified. For this to be the case, individuals must come to understand the value of "performing one action and not another" (Berger \& Luckmann, 1967, p. 93-94). 


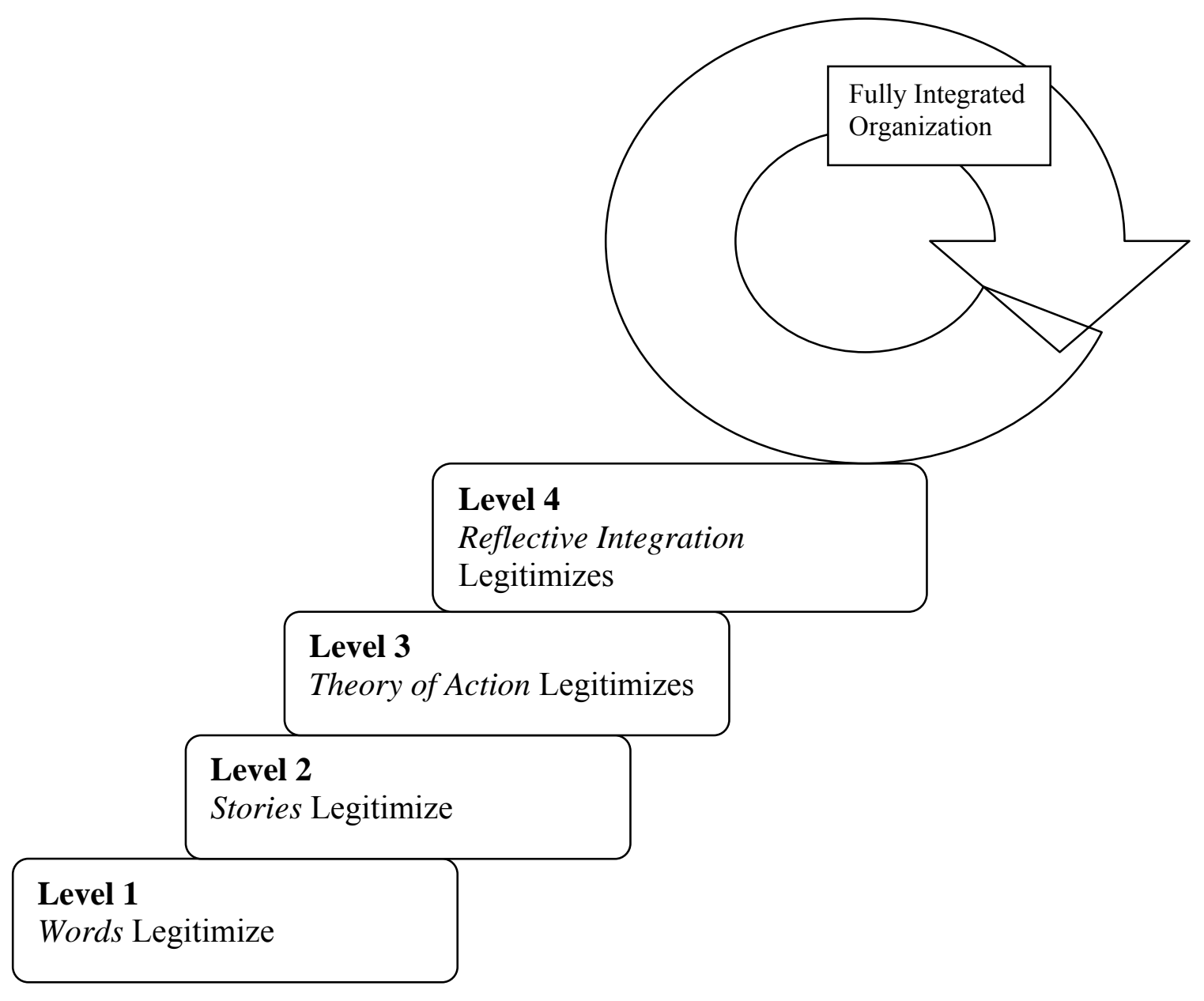

Figure 6. The four levels of legitimatization build upon one another sequentially, leading to a fully integrated organization.

The four levels of legitimization are all grounded in discourse. In level one, legitimation is present as soon "as a system of linguistic objectifications of human experience is transmitted" (Berger \& Luckmann, 1967, p. 94). In other words, an institutionalized process can begin its way towards legitimization simply by being named and discussed aloud. An example from the archival data utilized for this study illustrates this first level. Before the pilot program evaluation, stakeholders for this research were not accustomed to using the words stakeholders or logic model. By the time the program 
evaluation report was made to the superintendent's council, these words were in routine use by at least three groups of people: the council, the program evaluation stakeholders, and the district principals.

Level two moves beyond mere words and phrases and moves toward more complex narrative forms, like stories and moral maxims. Again, an example from the archival data serves to clarify this level. Once the pilot program evaluation concluded, people who participated spoke of sitting at the table. The program manager told numerous people the story of overcoming the anxiety she felt at the start of the evaluation. Another stakeholder explained to non-participants the unanticipated benefits of the experience.

Level three, legitimation, moves beyond stories and wise sayings and contains "explicit theories by which an institutional sector is legitmated in terms of a differentiated body of knowledge" (Berger \& Luckmann, p. 94). This phase is analogous to Patton's (1997) theory of action, or, in other words, "an articulation of what is supposed to happen in the process that is intended to achieve desired outcomes. The linkages between processes and outcomes are made explicit" (p. 223). It is in phase three that the "provinces of meaning" and "discrete processes of institutional order" begin to become integrated (Berger \& Luckmann, p. 96). To recap the connections between levels is to say that words progress into stories, which then provide the foundation for theory of action, which is also known as program rationale (Rossi, Lipsey, \& Freeman, 2004). These levels are progressively linked and are necessary before moving to level four.

Finally, at level four, reflectively integrative discourse allows the words, stories, and rationales of the first three levels to be integrated into the greater organizational 
mission and vision. This raises the process to the symbolic level. Bolman and Deal (2003) explain "the more complex a role structure (lots of people doing many different things), the harder it is to maintain a focused, tightly coupled enterprise" which raises the dilemma of "differentiation versus integration" (p. 69). The symbolic processes of level four legitimation offer a mechanism to redress the dilemma. It is on this level that ideas are integrated within organizational culture. Implementation of the evaluation tools developed as a result of this research would be one indication that The School District has reached the fourth phase of legitimation.

Double-loop learning. Morgan (1997) argues that a true learning organization must provide for double-loop learning. The UFE design of this research provides an avenue for stakeholders to strengthen the organization's self-questioning behaviors and, thus, "detect and correct errors in operating norms and thus influence the standards that guide detailed operations" (p. 86). According to Morgan, many organizations are good at single-loop learning, but few have developed the capacity for double-loop learning.

Discourse in Social Construction of Reality. Because objectivation, institutionalization, and legitimation are all dependent upon words and dialogue, and because the UFE design of this research is dependent upon discourse in a variety of settings, it is important to review the nature and role of language in the social construction of knowledge. Reason and Bradbury (2001) "suggest that the human world as we know it is produced and reproduced by the innumerable acts and conversations that we undertake daily" (p. 449). Bergman and Luckmann argue language grounds reality: "the common language available to me for the objectification of my experiences is grounded in everyday life and keeps pointing back to it even as I employ it to interpret 
experiences in finite provinces of meaning....[ie] I translate the non-everyday

experiences back into the paramount reality of everyday life" (p.26). Moreover, Bergman and Luckmann provide an overview of language which elucidates the connection between language and social construction of knowledge within context: "because of its capacity to transcend the here and now, language bridges different zones within the reality of everyday life and integrates them into meaningful whole. The transcendences have spatial, temporal, and social dimensions" (p. 39).

The importance of dialogue and conversations is widely noted in the literature (Baumgarter, 2001; Berger \& Luckmann, 1967; Bruffee, 1999; Lencioni, 2002; Merriam, 2001; Nonaka \& Takeuchi, 1995; Patton, 1997; Preskill \& Torres, 1998; Starratt, 2003). Bruffee believes "conversation... is of such vital importance to learning that with it, any of us has a shot at doing whatever we want to do. Without it, few of us stand a chance" (p. 14). He advocates for individuals, teams, and organizations to become truly collaborative and innovative in their approach to problems they will "have to learn to trust the power of conversation" (p. 18). Merriam writes that with dialogue and reflection, organizations can not only work toward solutions, but in so doing, produce sustained change. Baumgartner notes the "learning process involves questioning, critical reflection, and the need to engage in rational discourse [in order] to gain consensual validation for a new perspective" (p. 21). Nonaka and Takeuchi iterate the importance of articulation.

It is in finding ways to express the inexpressible that language pushes the boundaries of thought into the realm of innovation. Bruffee explains the "reacculturative, conversational process, direct and indirect, by which bearers of intellectual tradition [for example teachers and other professionals] construct knowledge in the langue of their 
communities of knowledgeable peers" (p. 54). He goes on to state that, indeed, "what we know is a conversational artifact...it means that knowledge is what we can explain to one another's satisfaction" (p. 118). Preskill and Torres' model for evaluative inquiry is predicated upon dialogue and enlarged via other pathways which are themselves largely, though certainly not exclusively, conversational: reflection, asking questions, identifying and clarifying values, beliefs, assumptions, and knowledge. Indeed, it is important to note that dialogue and conversation can, in fact, take written forms as well as spoken.

Written language is another vital expression of communication. It manifests its presence for individuals as both consumers and producers, much the same way verbal language does. Language materializes as text to be read. Bruffee (1999) explains "by reading, we acquire fluency in the language of the text and make it our own. ...conversely, we make the authors we have read members of our community" (p. 8). We encounter such texts in a variety of circumstances. Nonaka and Takeuchi (1995) iterate the variety of forms that language can take, both in the spoken and written form as it reifies knowledge: "schemata, mental models, beliefs, and perceptions...metaphors, analogies, concepts, hypothesis" (pp. 8,64). They explain that it is primarily through writing that language is able to convert tacit knowledge into knowledge that can be articulated. Starratt (2003) explores the relationship between knowledge, meaning, social activity and notes that when learners set out to inquire, to solve problems, it "involves multiple texts, experiments, and perspectives in the generating of meaning” (p. 37).

Electronic surveying. The function of this section is to provide an overview of the uses and applications of electronic surveying, particularly as it fits within the qualitative design of this study. There were two factors specifically related to this study which 
played into the use of electronic surveying. First, this technique was utilized as a mechanism to implement the Delphi technique. Second, it provided an opportunity to learn how it could be used by the organization as a mechanism to overcome geographical barriers. This strategy is predicated upon the understanding that language manifests itself in the electronic environment. Kilgore (2001) notes that this environment contributes to communities of practice, which are integral to building and disseminating knowledge within an organization (Nonaka \& Takeuchi, 1995). Richardson (2006) writes frequently on the uses of web tools and believes one of the biggest potentials of web tools is the ability to create spaces where people can collaborate with others electronically, arguing they are a "great way to manage and communicate the knowledge that gets created" (p. 30). The electronic survey is what Reason and Bradbury (2001) might appropriately deem a "new structure that allows for meeting in a community of practice [wherein] such, meetings are not easy [because of] our usually fragmented organizational structures" (p. 453). Certainly computers are the chief tool through which this language is shared. Bruffee (1999) explains:

The potential relationship between computers and education is quite another story to fulfill that potential, we have to understand the capacity of computers to foster reacculturative conversation among peers. That capacity results from the correspondence between the internal conversation of networked computers and how people learn. p. 111.

Organizational knowledge creation. The literature makes clear the organizational knowledge is socially constructed and, for the most part, context-based. The literature clearly defines knowledge as socially constructed (Bruffee, 1999; Flaherty, 1999; 
Merriam et al, 2001; Nonaka \& Takeuchi, 1995; Preskill \& Torres, 1999; Starratt, 2003). Bruffee explains the effect of working in concert with others to analyze a common text: "although we learned a lot from what we read, we learned a lot more from what we said to one another about what we read. Each of us began to change, and we discovered that the most powerful force changing us was our influence on one another" (pp. 8-9). Nonaka and Takeuchi elucidate the role social interaction plays in the creation and expansion of human knowledge. They vanquish the notion that organizational knowledge conversion can be confined within an individual. Rather they explain it is a "social process between individuals" (p. 61). Starrat reminds that it is not only knowledge, but ultimately meaning as well that is socially produced. Bruffee expands the definition to embrace not only knowledge and meaning, but also diagnostic judgment. Further he argues that in most cases there is no one architect of meaning, rather it evolves as a type of "community consensus" (p. 56). Preskill and Torres link all of this to the perpetual need to solve organizational problems and issues: inquiry and subsequent action become social and communal activities. The UFE design of this study paid particular attention to these precepts allowing for the community of stakeholders to participate individually and collectively in the construction of meaning.

Learning is intentional and contextual (Bruffee, 1999; Hansman, 2001; Kilgore, 2001; Marsick \& Watkins, 2001; Merriam, 2001; Nonaka \& Takeuchi, 1995; Preskill \& Torres, 1999; Starratt, 2003). Thus, it is critical to examine the role context plays in organizational knowledge creation and problem solving, and it is ill advised to apply "blinding focus on the individual learner whle ignoring the sociohistorical context in which learning occurs" (Kilgore, 2001, p.11). Hansman explains the link between social 
interaction and particular context: "the core idea in situated cognition is that learning is inherently social in nature. The nature of the interactions among learners, the tools they use within these interactions, the activity itself, and the social context in which the activity takes place shape learning" (p. 45). Furthermore, he believes the approach to adult learning is best addressed via situations, as opposed to subjects. Marsick and Watkins (2001) created a model that juxtaposes the larger social setting and the more specific learning context. They explain the model thus:

The circle in the center represents our belief that learning grows out of everyday encounters while working and living in a given context...the outer circle represents the context within which the experience occurs....the context for learning that plays a key role in influencing the way in which people interpret the situation, their choices, the actions they take and the learning that is effected (p. 29).

On the topic of interpretation, Flaherty (1999) puts forth a model for understanding how language and experience bound within a particular context can bring about changes in actions driven by interpretations. Preskill and Torres (1999) further iterate that the generation of organizational knowledge creation and knowledge involves "developing systems and structures that not only allow but also encourage organization members to learn and grow together- to develop communities of practice" (p. 14). Nonaka and Takeuchi (1995) tie all these ideas together by explaining both "information and knowledge are context- specific and relational in that they depend on the situation and are created dynamically in social interaction among people" (p. 59). 
The problem serving as impetus for this UFE was that The School District was unaccustomed to utilizing program evaluation in either the sense mandated by MSIP or in the sense of knowledge creation. Nonaka and Takeuchi (1995) assert that knowledge creation and, thus, organizational innovation, comes about in response to the pressure of current or impending problems and/or organizational issues. Merriam (2001) explains that one of the five assumptions underlying adult learning is a need for a problem and a subsequent interest in immediate application of knowledge. Bruffee (1999) notes that the impetus for effective collaboration is often a group's focus “on solving or dealing with a formidable problem" (p. 9). Preskill and Torres (1999) define evaluative inquiry, the workhorse of organizational knowledge creation, as "an ongoing process for investigating and understanding critical organizational issues" (p. 1). Preskill \& Torres (1999) note the importance of intentional and contextual learning. They argue that such a paradigm "involves developing systems and structures that not only allow but also encourage organization members to learn and grow together- to develop "communities of practice"" (p. 14). Berger and Luckmann (1967) explain how the social construction of reality causes organizations to address and integrate problems. "Even the unproblematic sector of everyday reality is so only until further notice, that is until continuity is interrupted by the appearance of a problem. When this happens the reality of everyday life seeks to integrate the problematic sector into what is already unproblematic" (p. 24).

\section{Summary}

The No Child Left Behind Act of 2001 requires K-12 organizations to perform numerous program evaluations, despite the fact few of these organizations have the professional capacity to support program evaluations beyond the minimum level required 
to achieve federal compliance (Rossi, Lipsey \& Freeman, 2004; Scriven, 1996).

Because the K-12 arena is not populated with professional program evaluators, evaluations typically emphasize outcomes at the expense of process assessment while also prioritizing compliance needs ahead of other stakeholder interests (Eddy \& Berry, 2007). As early as 2002, the Department of Education expressed concerns that "evaluation studies are not as helpful as they could be to practitioners at the local level, nor can they answer questions of causation" (New Directions, 2004).

A review of related literature for this study provided an overview of evaluation history, approaches, and applications, with a particular emphasis upon UFE model. The review of literature elaborated upon the ideas embodied within the social construction of reality. It also discussed electronic surveying before segueing into an overview of organizational knowledge creation. 


\title{
CHAPTER THREE
}

\section{RESEARCH METHODOLOGY}

\author{
Introduction
}

The No Child Left Behind Act (NCLB) of 2001 requires K-12 organizations to perform numerous program evaluations, despite the fact few of these organizations have the professional capacity to support program evaluations beyond the minimum level required to achieve federal compliance (Rossi, Lipsey, \& Freeman, 2004; Scriven, 1996). Because the K-12 arena is not populated with professional program evaluators, evaluations typically emphasize outcomes at the expense of process assessment while also prioritizing compliance needs ahead of other stakeholder interests (Eddy \& Berry, 2007). As early as 2002, the Department of Education expressed concerns that "evaluation studies are not as helpful as they could be to practitioners at the local level, nor can they answer questions of causation" (New Directions, 2004). The selected Missouri school district, hereafter referred to as The School District, has not yet determined how to implement program evaluations that are routinely capable of maximizing the use of stakeholder time, overcoming geographic constraints, and providing anonymity where necessary while still promoting organizational knowledge creation.

\section{Research Paradigm and Philosophy}

To best understand how processes and meanings evolve from any research, the reader is well-served to understand the cognitive logic of the researcher. Coghlan and Brannick (2005) emphasize "researchers' epistemological and ontological perspectives legitimate their own distinctive way of doing research and determine what they consider 
as a valid, legitimate contribution to knowledge or theory" (p. 5). This research is framed by a decidedly subjectivist ontological point of view which makes room for the idea that perception is reality. As such, reality hinges on individual cognition; it has multiple frames of reference- as opposed to an objectivist view that alternatively argues there is a single reality which hinges on external determinants.

Epistemology, the study of the theory of knowledge, concerns itself with questions regarding the essence and origin of knowledge. Again, the discipline is separated into objectivist and subjectivist leanings. Interpretation of language serves as a distinguishing factor. The researcher is an epistemological subjectivist, and, as such, "denies the possibility of a theory-neutral language" (Coghlan \& Brannick, 2005, p. 5). The confluence of these subjective stances creates an interpretivist/constructivst research stance. Additionally, it is important to contextualize this stance within the general paradigm of evaluation design, which Cronbach (1982) observed is "an exercise of the dramatic imagination" (p. 23). Finally, the research must be situated within the specific paradigm of the UFE approach, which is primarily judged, subjectively, by its utility to stakeholders (Patton, 1997).

\section{Problem and Purpose Overview}

The School District has not yet determined how to implement program evaluations that are routinely capable of maximizing the use of stakeholder time, overcoming geographic constraints, and providing anonymity where necessary while still promoting organizational knowledge creation. 
Problem Statement. The School District needs a program evaluation model that balances organizational knowledge creation with cost effectiveness, efficiency, and compliance issues.

Purpose of the Study. The purpose of this study was to conduct a UtilizationFocused Evaluation (Patton, 1997) of the program evaluation process of The School District. The study will provide The School District a site-specific process and model for future program evaluations.

\section{Research Questions}

To address the purpose of the study, the following research questions will guide the study:

1. Can a site-specific model for program evaluation be generated?

2. What data can be gathered to inform the creation of the program evaluation model?

3. How will data be analyzed to inform the creation of the model?

\section{Research Methods}

A qualitative case study methodology was used. According to Merriam (1998), a case study can provide a detailed examination and understanding of one setting, a single subject, or a particular event. Denizen and Lincoln (2000) note case studies can be a disciplined force in setting public policy and in generating reflection on human experience. The topic for this case study was selected in the hope that the result would inform The School District's future program evaluation practices. This case study was differentiated from other research in that it was developed via The School District's unique paradigm. This was accomplished using Utilization-Focused Evaluation (UFE) 
principles (Patton, 1997) in order to provide the basis for the evaluation of The School District's program evaluation model. The UFE paradigm suggests that for evaluations to be useful, and thus utilized, stakeholders must be involved as much as possible with all facets of the evaluation. The UFE approach stipulates that the primary intended users will participate in the selection of the most significant content, methods, theory, and applications pursuant to the contextual reality in which the organization functions.

This study was predicated upon qualitative research methods. The School District's superintendent, who articulated the need for a program evaluation model in her district, inspired this proposed research. She explained how the pervasive culture of compliance erodes away at any hope for organizational knowledge creation. She expressed an unwillingness to allow this to happen on her watch and explained that she would very much like to engage her key stakeholders in the challenge of engendering an organizational stance which eschews cow-towing to compliance and sacrificing learning on the altar of expediency (personal communication, December 16, 2007). When the circle of influence was broadened to include other stakeholders, her cabinet, the superintendent layered the aforementioned challenge upon the recently articulated district vision, mission, and goals, particularly: culture of collaboration, ensuring a safe learning environment, and fiscal responsibility. The stakeholders were particularly interested in learning how efficiency could be best integrated into program evaluation; this interest arose from a recent large-scale program evaluation which absorbed a high level of district resources- particularly in terms of building principals' time. In short, it became clear that the stakeholders of this proposed research desired a transportable model for program evaluation, one which functioned within the contextual constraints of The School 
District's vision, mission, and goals, but which was flexible enough to utilize on a variety of program evaluations.

\section{Setting and Population}

The School District selected for the case study was chosen because of its desire to pursue a study of program evaluation options culminating in some type of site-specific, transportable model for k-12 program evaluation. The superintendent and her cabinet were aware of the need to locate a model for program evaluation that would provide flexibility for a compendium of evaluation purposes. Moreover, The School District is noted for its open desire to pursue organizational knowledge creation, a facet of the culture promoted by its superintendent whose educational background stems from a unique mixture of leadership, policy analysis, and program evaluation.

\section{Research Path}

This case study was conducted as a Utilization-Focused Evaluation. As such, it is designed to involve the intended users in evaluating and determining the utility of results (Patton, 1997). The superintendent has requested a study of The School District's program evaluation process. Moreover, the superintendent requested that the Utilization-Focused Evaluation approach be employed. See Figure 5 for an illustration of the research path for this study.

The UFE was initiated via an interview (Appendix D) of the primary intended user, the superintendent, to determine the specific direction of the study and the program's greatest need (Figure 5). Questions were framed in terms of vision and anticipated outcomes to allow for rich, thick description as recommended by Merriam (1998). In keeping with the UFE paradigm (Patton, 1997), questions for the interviews 
with other stakeholders (Appendix E) will emerge from this opening encounter. The other stakeholders for this study are defined as the Superintendent's Cabinet. In addition to the superintendent, the cabinet consists of the eight administrators who lead the district's chief areas of service: Curriculum, Instruction, and Assessment; Elementary Education; Secondary Education; Human Resources; Community Relations; Finance; Special Education; and Technology and Operations.

This study was based upon qualitative data. The qualitative data came from current and archival sources. Content analysis was performed on federal, state, and local documents; data was reduced using an affinity diagram. Data from The School District's pilot program evaluation was analyzed, via content analysis, and reduced utilizing an interrelationship diagraph. As previously mentioned, interview questions were used to interview the superintendent and her cabinet (Appendices D, E, G). Following each interview, the tapes were transcribed to provide written text for analysis. According to Seidman (2006) "the primary method for creating text from interviews is to tape-record the interviews and to transcribe them. Each word a participant speaks reflects his or her consciousness" (p. 114). In addition to the face-to-face interviews, an electronic survey, constructed with open-end questions, was delivered to the stakeholders via an email link (Appendix F). Again, in keeping with the UFE design, questions for the electronic survey emerged from early research stages in an effort to ensure maximum utility of information for the intended users (Patton, 1997). All data was collected and triangulated to provide feedback, in the form of a recommended model for program evaluation, to the intended users for evaluation, the superintendent and her cabinet. 


\section{Data Collection Strategies}

This study involved data collection in several forms: gathering of pertinent documents, archival data, long and short interviews, electronic surveys, and, potentially, a follow-up review of literature. All interviewees were informed that the information obtained was for the evaluation of The School District's program evaluation process with the intention of creating a transportable, site-specific model for program evaluations within The School District in the future. Prior to the start of research, a letter requesting permission to conduct the study within The School District (Appendix B) was sent to the superintendent. A letter of consent was obtained from the superintendent. All stakeholders were informed that any information gathered was used for the purposes of this research study; each signed an agreement of informed consent (Appendix C). Five data collection mechanisms were used (Figure 7).

Long interviews. The researcher developed interview questions for the primary intended user (Appendices D and G). Two interviews, taking approximately 60 minutes each, were undertaken and digitally recorded. A research assistant transcribed the recording. Member checking of the transcripts occurred. Revisions were made, as called for, as a result of the member check. None of the revisions required more than revising a few words that the transcriptionist misheard.

Document analysis. The No Child Left Behind Act of 2001, Missouri School Improvement Program (MSIP)- Cycle 4, The School District's board policy, and The School District's vision, mission, and goals were gathered.

Archival data. The program evaluation data and material generated from The School District's full-scale evaluation pilot was used. 
Short interviews. Development of questions for the short interviews (Appendix E) was guided by issues raised during the first long interview with the primary user. Other stakeholders were defined as the Superintendent's Cabinet. In addition to the superintendent, the cabinet consists of the eight administrators who lead the district's chief areas of service: Curriculum, Instruction, and Assessment; Elementary Education; Secondary Education; Human Resources; Community Relations; Finance; Special Education; and Technology and Operations. These interviews took approximately 20 minutes and were digitally recorded, as per Seidman (2006) and then transcribed by the research assistant. Member checking of the transcripts occured. Revisions were made, if called for, as a result of the member check.

Electronic survey. Feedback from stakeholders and the process of the Delphi technique informed the development and implementation of questions for the electronic survey (Appendix F). The Delphi technique "aggregates the judgments of a number of individuals through a series of [questions] ...to which individuals respond anonymously" (Garavalia \& Gredler, 2004, p. 377). The conceptual underpinnings of objectification, instituationalization, and legitimation acted as a filter, serving to categorize the emergent data within the survey, which was constructed using Fink's (2006) steps for surveys. The survey was powered by Survey Monkey (2008), which is approved by MU IRB for research purposes. In addition to the written informed consent form, the email invitation to participate in the electronic survey included assurances of confidentiality (Appendix $\mathrm{H})$.

Follow up review of literature. Process use surfaced as an unanticipated emergent theme. A secondary review of literature was undertaken to shed light on the implications. 


\section{Data Analysis}

This study utilized several mechanisms for data analysis and reduction: content analysis, open and axial coding, affinity diagramming, and interrelationship diagraphing.

Long interviews. Open and axial coding was utilized on the long interview transcripts. Denzin and Lincoln (2005) explain "coding is the first step in taking an analytic stance toward the data" (p. 517.) Both long interviews will serve the UFE design by ensuring that the primary intended user's concerns are addressed. The second interview will specifically function as the lens for "interpreting evaluation findings" (Stufflebeam and Shinkfield, 2007, p. 233).

Document analysis. Content analysis was performed on the No Child Left Behind Act of 2001, Missouri School Improvement Program (MSIP)- Cycle 4, The School District's board policy, and The School District's vision, mission, and goals. Merriam (1998) notes that qualitative content analysis is different from conventional content analysis in that it is a flexible process, one in which the researcher can analyze with the intent of understanding theoretical relationships. For this study, the content analysis will strive to elucidate connections between compliance mandates and the district vision, mission and goals. The data reduction will result in an affinity diagram (Brassard, 1996).

Archival data. Content analysis was employed on the program evaluation data and material generated from The School District's full-scale evaluation pilot. Thereafter, an interrelationship diagraph (Brassard, 1996) was employed to reduce the data. This process allowed the researcher to map logical patterns that emerged from the information. Because it does not require linear thinking, this technique will allow for the use of 
multidirectional thinking to integrate complexities and account for interrelationships (Brassard, 1996).

Short interviews. Open and axial coding were used on the short interview. transcripts.

Electronic survey. Open and axial coding were used on the survey results.

Follow-up review of literature. To create an additional filter for data reduction, content analysis and synthesis was employed. 


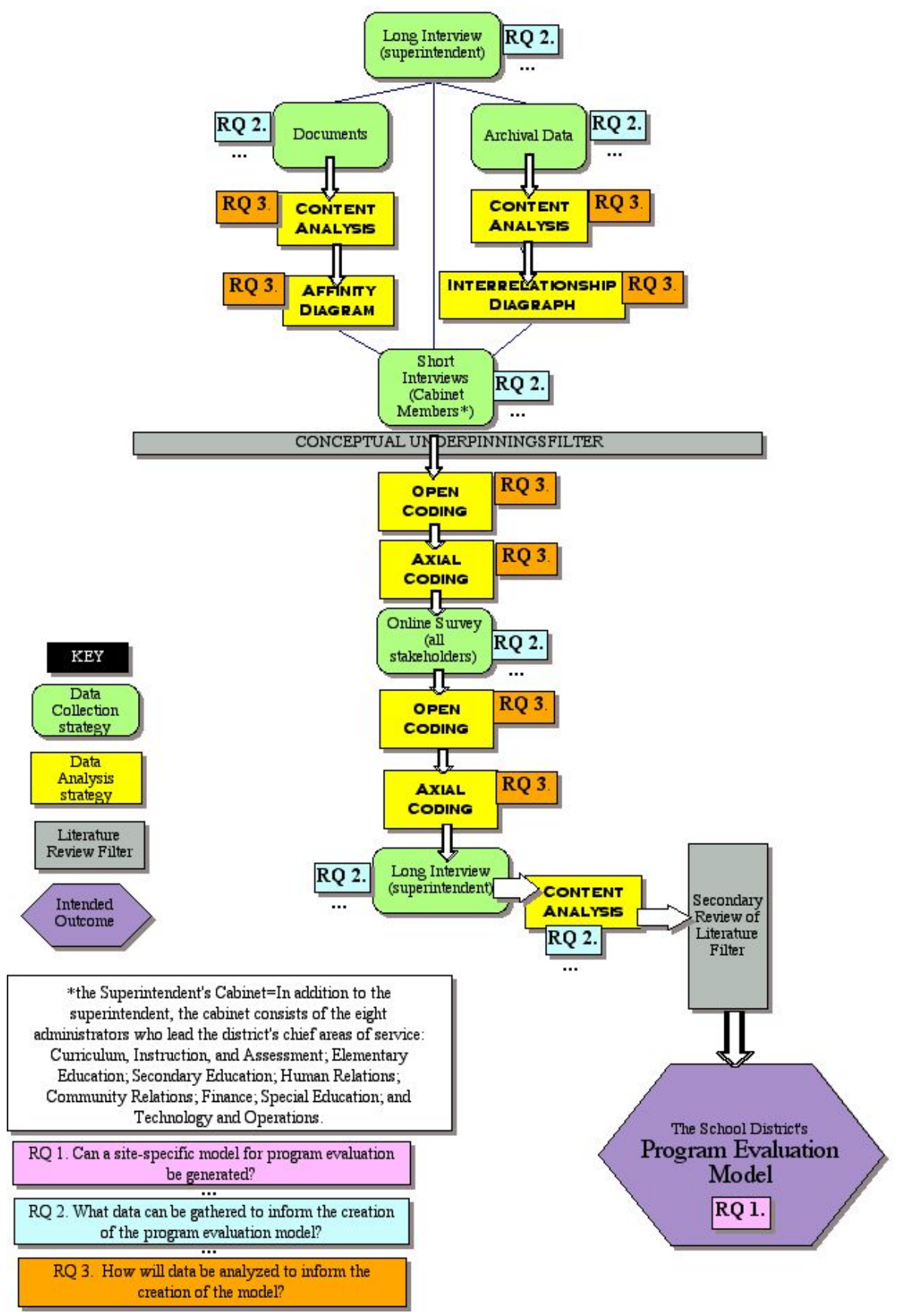

Figure 7. Reified data reduction process for this study. 


\section{Summary}

The School District had not yet determined how to implement program evaluations that are routinely capable of maximizing the use of stakeholder time, overcoming geographic constraints, and providing anonymity where necessary while still promoting organizational knowledge creation. The School District needed a program evaluation model that balances organizational knowledge creation with cost effectiveness, efficiency, and compliance issues. The purpose of this study was to conduct a Utilization-Focused Evaluation (Patton, 1997) of the program evaluation process of The School District. The study provided The School District a site-specific process and a model for future program evaluations. 


\section{CHAPTER FOUR}

\section{FINDINGS}

\section{Introduction}

The School District had not yet determined how to implement program evaluations that are routinely capable of maximizing the use of stakeholder time, overcoming geographic constraints, and providing anonymity where necessary while still promoting organizational knowledge creation. The School District needed a program evaluation model that balances organizational knowledge creation with cost effectiveness, efficiency, and compliance issues. The purpose of this study was to provide a Utilization-Focused Evaluation (Patton, 1997) of The School District's program evaluation process. The study provided The School District a site-specific process and model for program evaluations in the future.

This evaluation was designed to provide a program evaluation model for The School District. The model provided the district with a transportable process, one which can be used on future program evaluations to support several non-exclusive outcomes: compliance with federal, state, and local mandates, process analysis, and organizational knowledge creation. As such, this study may have an impact on future programming within The School District. Because the model is transportable, this evaluation might also be of benefit other organizations within the K-12 arena.

A qualitative case study methodology was used. According to Merriam (1998), a case study can provide a detailed examination and understanding of one setting, a single subject, or a particular event. Denizen and Lincoln (2000) note case studies can be a disciplined force in setting public policy and in engendering reflection on human 
experience. The topic for this case study was selected in the hope that the result would inform The School District's policy and methods regarding program evaluation. This case study was differentiated from other research in that it was developed via The School District's unique paradigm. This was accomplished using Utilization-Focused Evaluation (UFE) principles (Patton, 1997) to provide the basis for the evaluation of The School District's program evaluation model. The UFE paradigm suggests that for evaluations to be useful, and thus utilized, stakeholders must be involved as much as possible with all facets of the evaluation. Utilization-Focused Evaluation involves the primary intended users in the selection of the most significant content, methods, theory, and applications based upon contextual reality.

The UFE was initiated via an interview (Appendix D) of the primary intended user, the superintendent, to determine the specific direction of the study and the program's greatest need. Questions were framed in terms of vision and anticipated outcomes to allow for rich, thick description as recommended by Merriam (1998). In keeping with the UFE paradigm (Patton, 1997), questions for the interviews with other stakeholders (Appendix E) emerged from this opening encounter. The other stakeholders for this study were identified as the Superintendent's Cabinet, which includes the eight district administrators who lead the district's services: Curriculum, Instruction, and Assessment; Elementary Education; Secondary Education; Human Resources; Community Relations; Finance; Special Education; and Technology and Operations.

This study was based upon qualitative data. The qualitative data came from current and archival sources. Content analysis was performed on federal, state, and local documents; data were reduced using an affinity diagram. Data from The School District's 
pilot program evaluation were analyzed, via content analysis, and reduced utilizing an interrelationship diagraph. As previously mentioned, questions were used to interview the superintendent and her cabinet (Appendices D, E, G). Following each interview, the tapes were transcribed to provide written text for analysis. According to Seidman (2006) "the primary method for creating text from interviews is to tape-record the interviews and to transcribe them. Each word a participant speaks reflects his or her consciousness" (p. 114). In addition to the face-to-face interviews, an electronic survey, constructed with open-end questions, was delivered to the stakeholders via an email link (Appendix F). Again, in keeping with the UFE design, questions for the electronic survey emerged from early research stages, specifically data analysis of interviews, in an effort to ensure maximum utility of information for the intended users (Patton, 1997). All data were collected and triangulated to provide feedback, in the form of a recommended model for program evaluation, to the intended users for evaluation, the superintendent and her cabinet.

This study involved data collection in several forms: gathering of pertinent documents, archival data, long and short interviews, electronic surveys, and, potentially, a follow-up review of literature. All interviewees were informed that the information obtained was for the evaluation of The School District's program evaluation process with the intention of creating transportable, site-specific model for program evaluations within The School District in the future. Prior to the start of research, a letter requesting permission to conduct the study within the School District (Appendix B) was sent to the superintendent. A letter of consent was obtained from the superintendent. Stakeholders were informed that any information gathered was used for the purposes of this research 
study; each signed an agreement of informed consent (Appendix C). Five data collection mechanisms were used: long interviews, document analysis, archival data analysis, short interviews, and an electronic survey. This chapter will report the results of each of these research activities. This chapter is organized into several sections: introduction, first long interview, document content analysis, archival data content analysis, short interviews, electronic surveys, second long interview, original research questions, and summary.

\section{First Long Interview}

The primary intended user of the evaluation findings was identified as the superintendent of The School District. Therefore, research began with the first step in the data collection sequence-- a forty-five minute interview that took place in her office. The interview was tape-recorded to "ensure everything said [was] preserved for analysis" (Merriam, 1998, p. 87). A research assistant not affiliated with The School District then transcribed the tape verbatim the same day. The transcript was returned immediately upon completion to the interviewee who confirmed its accuracy via member-check as recommended by Merriam. The rapid turnaround ensured that the interviewee would have the experience fresh in her memory when she reviewed the transcript. In addition to the tape recording, notes were taken during the interview to record researcher thoughts and reactions.

The first long interview was driven by two questions: (a) What is your vision for program evaluation in your school district? (b) What are the anticipated outcomes from evaluation in your mind? The superintendent confirmed the fact that she was the primary intended user of this study of the evaluation model. She identified the members of her cabinet as the major stakeholders. She stated that she believed this Utilization-Focused 
Evaluation was needed so the district could obtain a site-specific model for program evaluation. During the course of the interview, she emphasized why she believed it was critical for the stakeholders to agree upon a model of program evaluation for The School District. She said, “what I'm finding is, we don't have very often, intense focus or purposes and we definitely in this organization don't have agreed upon processes. Definitely don't. And if I were to define what I think one of the greatest weaknesses of this organization right now is we don't have agreed upon processes, which means we are still in reactive mode. Until we get out of reactive mode, we're going to make haphazard decisions that are not going to be hitting the target" (Long interview, March 18, p. 14).

Two types of coding, open and axial, were used. Open coding of the long interview revealed several priorities. Words that appear often included mechanism, components, comparative data, stakeholders, ownership, knowledge creation, conflict resolution, systemic, logic modeling, purpose, process, right reasons, data, efficient, and effective. The impassioned delivery of these words within context illustrated the weight the superintendent put on the concepts. Axial coding of the long interview was organized according to several strands: outcomes for this particular program evaluation, outcomes for a fully operational program evaluation operation, and a vision for the role program evaluation could play in transforming organizational culture.

She verbalized her anticipation regarding outcomes from this particular program evaluation. She hoped that the UFE would bring about the creation of a mechanism to make informed programming decisions. She also believed this mechanism would be in the form of a consistent model, probably containing three sections: part one to contain "commonalities of expectations of any program" (Long interview, March 18, p. 3). She 
hoped the model would provide a path for comparative data analysis, especially if it contained a unique section designed in conjunction with the program manager. She wanted a concrete plan for stakeholder integration- a "means by which we can do multiple evaluations and still have them thorough[ly]" grounded by stakeholder input (Long interview, March 18, p. 11). Finally, she hoped that the would "automatically support our values of culture of collaboration....commitment to excellence.....and integrity of actions" (Long interview, March 18, p. 7).

Beyond the scope of this particular UFE and looking towards the future, the superintendent anticipated several significant outcomes from a fully operational program evaluation system. These include

1. the ability to implement the aforementioned mechanism to make informed programming decisions, particularly relative to implementation of change initiatives.

2. a greater understanding of the value and impact various programs have on the ability of The School District to accomplish its stated mission, educating each child for success.

3. increased ownership in district programming.

Her vision for program evaluation centered on transforming culture, a theme which presented itself several ways. She believed that program evaluation is capable of

1. engendering evaluation capacity at all levels of the organization, particularly through the construction and use of logic modeling.

2. casting off the airs of reactivity in favor of proactivity as an organizational stance. 
3. "creates new knowledge that can be a springboard into a rich program, better services to kids, better practices" (Long interview, March 18, p. 8).

4. allowing questions to be posed without being perceived as insults or threats.

5. providing "a means by which we can move toward that benchmark we call excellence" (Long interview, March 18, p. 10).

The interview ended with the agreement regarding which documents would be analyzed, how the archival data would be studied, and which questions were most vital for the other stakeholders to answer.

\section{Document Content Analysis}

Content analysis of important documents pertinent to this study was performed on the No Child Left Behind Act of 2001, Missouri School Improvement Program (MSIP)Cycle 4, The School District's board policy, and The School District's vision, mission, and goals. These documents were analyzed to assist the researcher in understanding the overt and theoretical relationships among the documents (Appendix I).

An analysis of the complete No Child Left Behind Act of 2001 document illuminated the pervasive nature of the evaluative task in K-12 education (Appendix K). One important aspect of the document analysis involved studying the frequency and dispersion of the word evaluation with the intent of understanding the scope of evaluation within the legislative mandate. Specifically, the term evaluation is mentioned 311 times, a frequency determined by converting the document to a Microsoft Word file and performing a word search via the "find word" option. Use of the word evaluation is evenly spaced throughout the six sections of the 10 titles in the legislation. This was determined via the creation of a workbook designed to separate evaluation mandates 
according to page number, section, part and title of the law. See Appendix $\mathrm{N}$ for an example of the analysis workbook. It is important to note the contexts in which evaluation is framed shift throughout the document. These shifts are important insofar as they encompass a variety of evaluation modes, for instance external vs. internal, taskssuch as analysis, reporting, and information dissemination- and topics: reading improvement programming, comprehensive school reform, language instruction for limited English proficient and immigrant students, $21^{\text {st }}$ century schools, and parent involvement policy and programming, to name a few.

An analysis of the Missouri School Improvement Program (MSIP) Cycle 4 revealed that program evaluation is treated in a centralized manner (Appendix $\mathrm{J}$ ). Unlike the recursive mentioning and mandating present throughout NCLB, MSIP Cycle 4 localizes program evaluation within one section where it calls for several activities: (a) biennial review of goals and objectives (b) report on the effectiveness of each program and service (c) documentation of action to "ensure that these programs efficiently achieve their goals." Moreover, each district is mandated to have a board- approved procedural plan that "coordinates the evaluation of all programs and services." There are four elements that must be present within any school district's evaluation plan:

1. Program goals and objectives for the programs and services offered;

2. Evaluation criteria and procedures for the programs and services offered;

3. Designated responsible persons for the programs/services;

4. List of programs due for evaluation and timelines for reporting the results of the evaluations to the board (MSIP, $4^{\text {th }}$ cycle). 
The School District does not currently have an evaluation plan in place as stipulated by MSIP, cycle 4.

An analysis of The School District's board policy brought to light information notably relevant to this study (Appendix L). There are eleven sections that cover the entire operation of The School District. Within the subsets of these eleven headings, evaluation is mentioned four times. Three of the four citations are found within Section A- Foundations and Basic Commitments: evaluation of school board operational procedures (policy AFA), evaluation of support staff (policy AFD), and evaluation of instructional programs (policy AFE). The fourth citation is within Section C- General School Administration: evaluation of the superintendent (policy CBG). None of these references particularly indicate program evaluation as conveyed professionally within NCLB or procedurally within MSIP, cycle 4. Policy AFA stipulates the school board shall review and continuously study its own operations. Policy AFD obviously directed towards performance reviews meant to be couched through the human resources department: evaluation of support staff. Policy AFE, evaluation of instructional programs, is articulated within this section specifically in terms of curriculum review protocols.

After analyzing board policy sections for the frequency and location of evaluation, the next step was to study the subheadings for references to programs. Subtitles within sections reflect the word "program" is utilized eighteen times over the course of six sections. Notably, 56 percent, or ten of the eighteen, references are within Section I: Instruction. 
The School District's vision, mission, and goals have implications for program evaluation as well (Appendix M). The mission is educating each child for success. The vision, a clause punctuated with a colon, most notably states a great place to learn. Though not explicitly articulated as such, the three goals function as mechanisms to qualify how The School District intends to achieve the vision: (a) create a learning environment that will produce academic success for all students (b) provide a safe, secure learning environment (c) practice fiscal stewardship of resources. Strategies, measurements, and action steps undergird each goal. None of these stipulate program evaluation.

The important finding was in the juxtaposition of the federal, state, and local perspectives (Appendix I). A summary list of the various responses elicited to a search for the program was generated (Appendix $\mathrm{O}$ ) and presented to the primary intended user. The most significant finding was that The School District has not embraced a coherent and/or site-specific definition of the word program, particularly as this word relates to the imperative of program evaluation. However, despite a lack of coherence, the analysis drew forth the fact that the way School District board policy references programs strongly resonates with the manner in which the No Child Left Behind Act of 2001 references policy. Another important concept that emerged is that at the LEA (local educational agency) level, in this case The School District, no concern is appropriated towards the methodological imperatives set forth for program evaluation as embodied in the federal legislation, particularly regarding edicts concerning randomized controlled trials. No evidence collected suggests The School District has the intent of pursuing such methods. 


\section{Archival Data Content Analysis}

Content analysis of archival data from The School District's pilot program evaluation, an evaluation of the English Speaker's of Other Languages program, was performed. The content analyzed included field notes from commissioning activities with district administration, one-on-one interviews and focus groups, stakeholder meeting agendas, a binder of best ELL practices, drafts of the evolving program logic model, final logic model, emails from the program manager to program evaluation staff, draft report of evaluation, and report submitted at final stakeholders' meeting. A process was used to identify key topics, as recommend by Merriam (1998). Specifically, the data were distilled via the process of constructing an interrelationship diagraph. The purpose of the interrelationship diagraph is to take a "central idea, issue or problem and map out the logical or sequential links among related items. It is a creative process that shows every idea can be logically linked with more than one other idea at a time" (Brassard, 1996, p. 41). This process was organized into three steps: identifying and articulating key ideas, organizing the ideas according to obvious trends, creation of relationship arrows.

All documents were organized according to date of creation and place within the evaluation timeline. All documents were then read and key topics and relevant characteristics were highlighted. Identified elements were then transcribed into one-line statements. Utilizing the computer program Inspiration, each statement was copied and pasted into a box; this resulted in a representation of what Brassard (1996) deems an "explosion of ideas" (p. 41). 


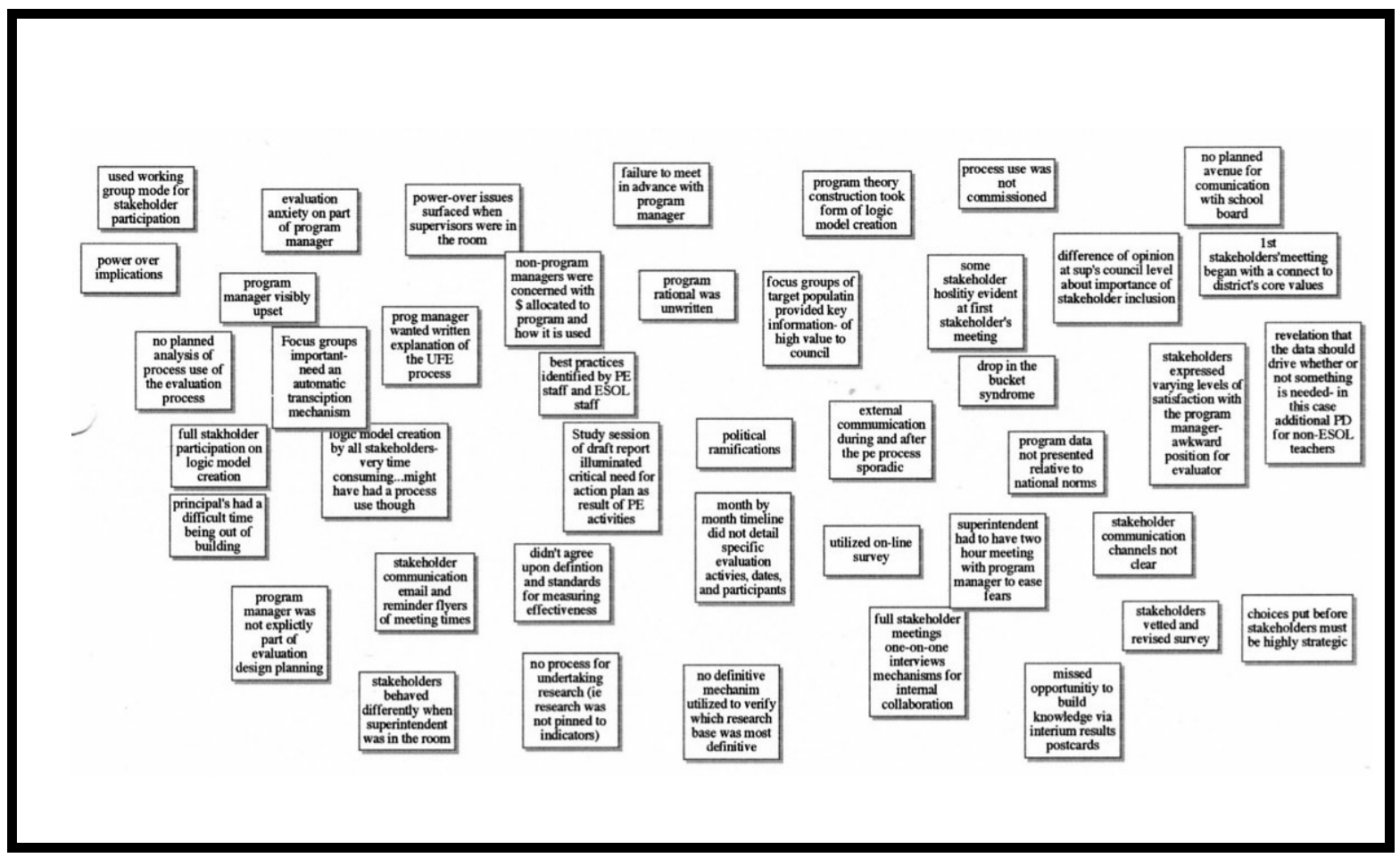

Figure 8. Step 1- Important concepts distilled from archival data result in the explosion of ideas (Brassard, 1996).

Once this step was complete, the next step was to analyze the ideas in search of recurrent patterns or trends. At this stage, the screen was printed and multi-directional arrows penciled in to identify potential relationships among ideas. Though this process was untidy, in keeping with Brassard's (1996) directions, it became clear that the individual cards were fraught with implications that could be categorized according to Bolman and Deal's (2003) four frames: human resource, political, symbolic, and structural. Furthermore, some of the individual cards carried implications for more than one frame; in such cases, the card was copied so that it could be categorized within as many frames as was appropriate, a key tenet of interrelationship diagraphing. It also became apparent that there were significant concepts that did not clearly fit within one of Bolman and Deal's frames because such ideas were closely connected to evaluation 
procedures, inclusive of stakeholder behaviors during the process. Because these statements were crucial to the evaluative process, a new category was created to indicate important concepts directly related to evaluation protocols, procedures, and behaviors: this category was entitled procedural.

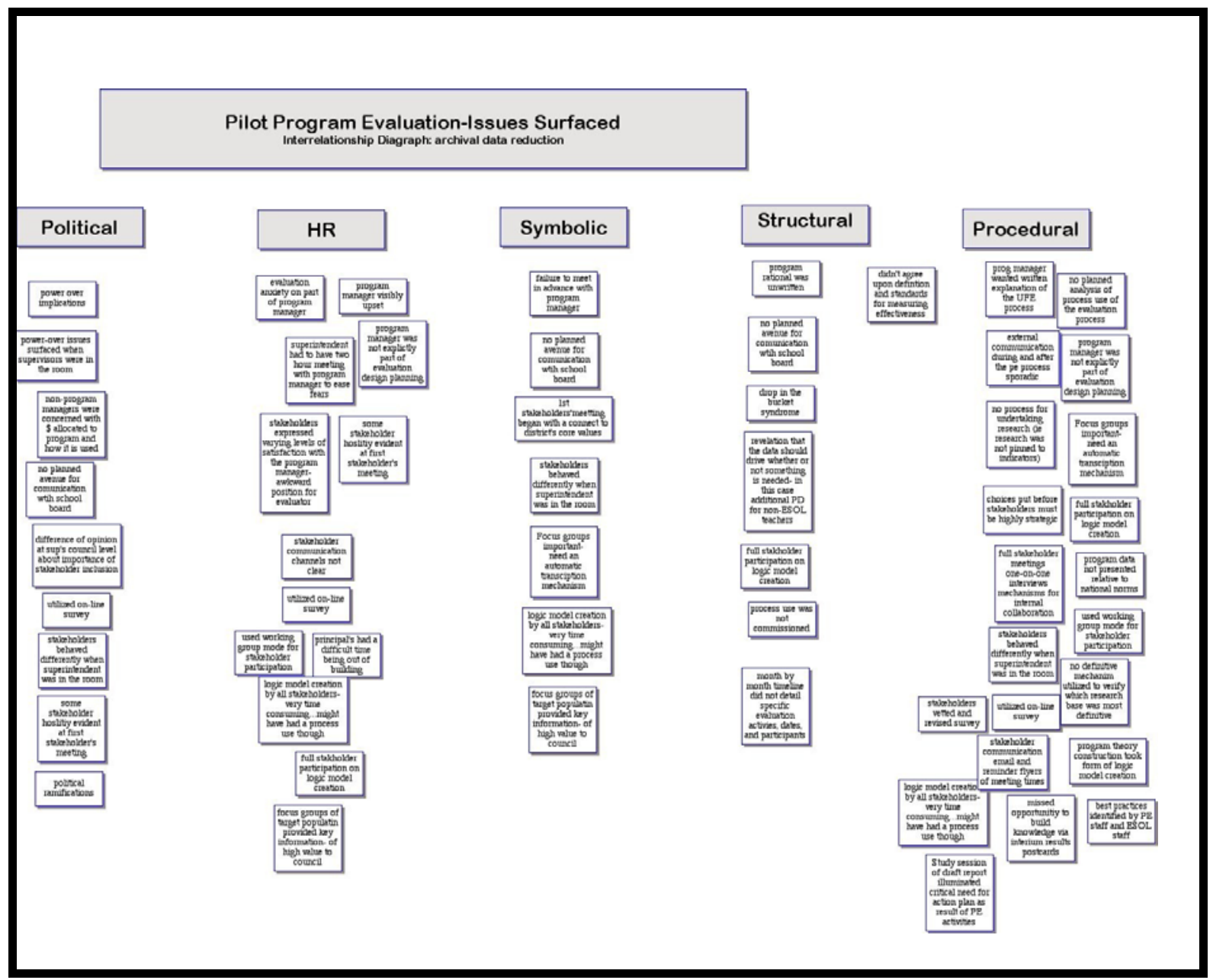

Figure 9. Step 2- Ideas organized according to Bolman and Deal's (2003) frames.

Once the cards were arranged according to frames, it was necessary to consider how the various cards implicated or impacted the others. To do this, arrows were once again penciled into the emerging diagraph. The result, though less chaotic than previous attempts, is still messy. 


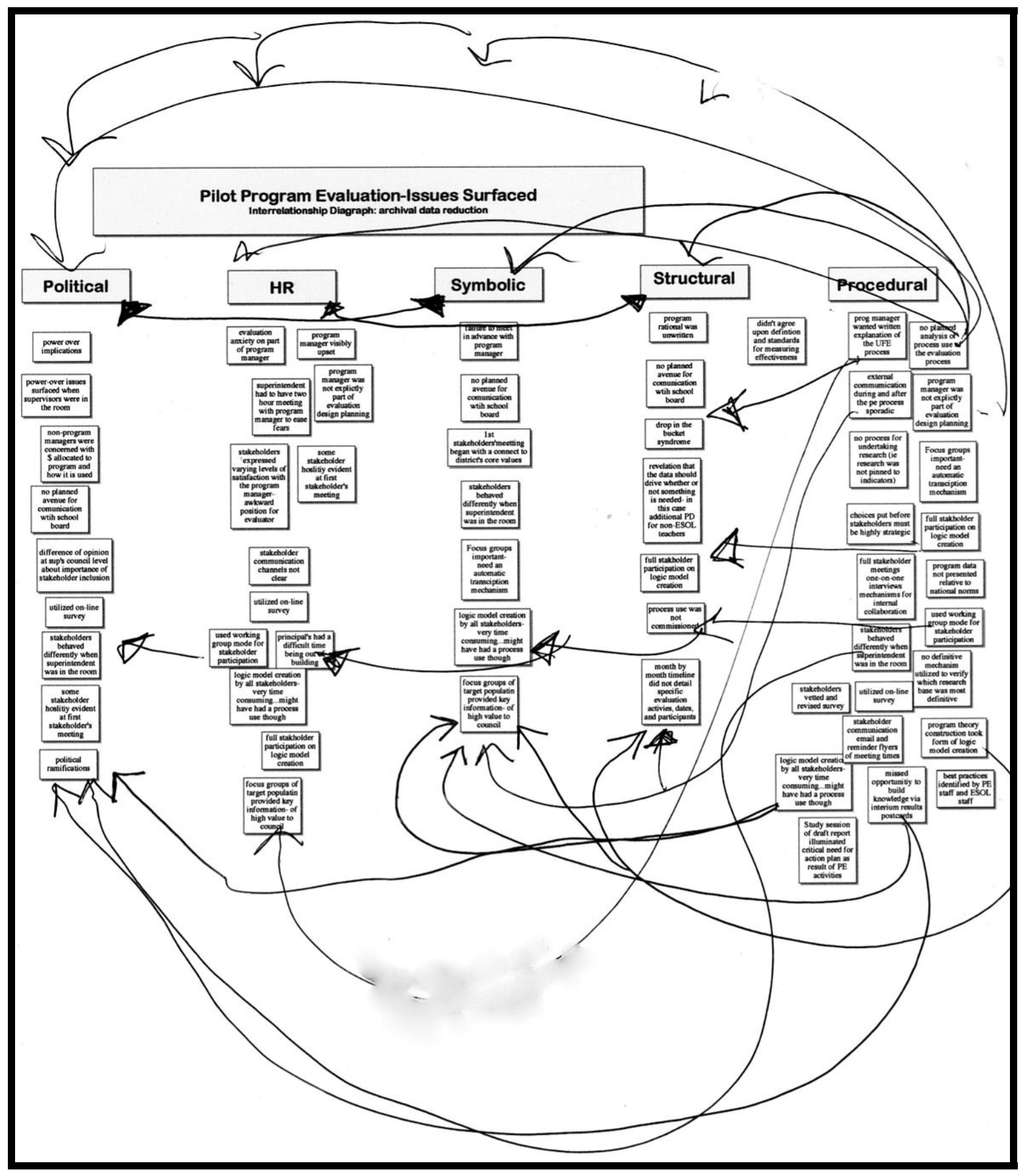

Figure 10. A picture of "outgoing arrows dominant" (Brassard, 1996) facilitates an understanding of the relationships among important ideas.

At this stage of the analysis, it became clear that the arrows were becoming less multi-directional. In fact, it became apparent that procedural issues had what Brassard 
(1996) terms "outgoing arrows dominant" (p. 62) and as such were begetting implications across all four of Bolman and Deal's (2003) frames. The resulting graphic, the product of an untidy process, distills meaning and helps to clarify understanding by focusing attention on cause and effect.

Pilot Program Evaluation-Issues Surfaced Interrelationship Diagraph: archival data reduction

\section{Procedural} Issues

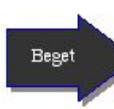

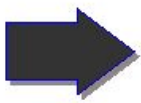
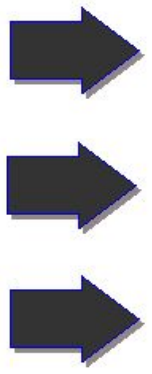

HR Implications

Political Implications

Symbolic Implications

Structural Implications

Figure 11. Procedural issues function as a basic cause which begets other implications.

According to Brassard, when dominant outgoing arrows are present, it means that a "basic cause, if solved, will have a spillover effect on a large number of items" (p. 62). Utilizing the interrelationship diagraphing process for analysis of archival data from The School District's Pilot Program Evaluation brought to light the manner in which procedural issues beget human resources, political, symbolic, and structural implications. Therefore, the data reduction process brought to light an important consideration: procedural issues, if solved, can bring about improvements and benefits to the organization, particularly in terms of human resources, politics, symbolism, and structure. 


\section{Short Stakeholder Interviews}

The next step in the data collection sequence was to conduct short, defined as no longer than 20 minutes, interviews with each of the other stakeholders, all members of the superintendent's cabinet. As with the first long interview, these interviews were taperecorded, to "ensure everything said [was] preserved for analysis" (Merriam, 1998, p. 87). A research assistant not affiliated with The School District then transcribed the tape verbatim within two days of the interviews. Upon receipt, the transcript was returned immediately to the interviewee who confirmed its accuracy via member-checking, as recommend by Merriam. The rapid turn-around ensured that the interviewee would have the experience fresh in his or her memory when reviewing the transcript. In addition to the tape recording, notes were taken during the interview to record researcher thoughts and reactions. Open coding was performed in the following manner

1. Transcript confirmed by interviewee

2. First close reading- highlighting and making note of possible trends

3. Second close reading- open coding and tallying of words that came out as trends during first close reading

4. Open coding workbook (Appendix I) creation

5. Utilized find feature in Microsoft Word to confirm hand counts of word frequencies

6. Removed all researcher comments from transcripts so as not to skew the find process 
7. Analyzed for words identified during $2^{\text {nd }}$ close reading and entered the frequencies into the Open Coding workbook

This open coding process illuminated important ideas as embodied in high frequency words. The most important concepts according to the frequency evidenced in the short stakeholder interviews were effectiveness (52), stakeholders (38), students (33), resources (23), and change (21). The only word to surface in every interview was effective.

After the open coding process was complete, a third close reading was used to begin the process of axial coding. Three main themes, all of which in some way took into account ideas evidenced via open coding, emerged: engendering collaboration, return on investment, and process use. Once it was determined these themes were present, each was highlighted throughout the text of the interviews utilizing yellow, green, and pink highlighting respectively (Appendix P).

Collaboration. Repeatedly throughout the short interviews stakeholders expressed the importance of bringing people together to work and think. One stakeholder explained, people need to feel validated and know that "we are all in this together" (Interview, March 19, p. 2). Another stakeholder noted people must "have input and participate in development" (Interview, March 18, p.2). Top down delivery does not foster solid implementation according to yet another (Interview, March 19, p.2). A different stakeholder for this study explained that stakeholders for various programs across the district deserve to be at the table working together (Interview, March 20). Another elaborated: "program evaluation can bring people around the table, allowing them to really talk to each other, to appreciate differences, and experience, as result, a change in perspective and accomplish what they might not on their own" (Interview, March 20). 
This stakeholder went on to say that this type of collaboration helps people feel better about being accountable... because people want to "be right when somebody comes and looks" at their program (p. 6).

In an off transcript comment, she later elaborated that people simply need guidance in understanding what it means to be right in this sense and that working together produces a confidence in the final product which generates this sense of rightness. Another stakeholder explained "there are many, many stakeholders involved in The School District that can really contribute" if only they knew how. This lets them know and opens up an avenue for their participation. He continued "we all make up the district and we all have the same goals, but for all the entities to work in concert with each other they need to know their strengths [and] where they fit in the district as a whole" (Interview, March 26, p. 4). These observations are supported by Bolman and Deal's (2003) assertion: “people's skills, attitudes, energy, and commitment are vital resources that can make or break an enterprise" (p. 114). The theme of collaboration as it has surfaced within these interviews supports the importance of fostering this element as an integral part of the district's program evaluation ideals.

Return on investment. Another emergent theme was the importance of return on investment. There was a common thread regarding the fact, expressed by a stakeholder, that The School District has "limited precious organizational resources. They need to be diverted to and used as effectively as you can... so you really need to [know] where are we spending most of our money and are we getting value out of it" (Interview, p. 2). Additionally, a different stakeholder spoke of the imperative of doing everything possible to express in word and deed that The School District takes stewardship of resources 
seriously. Every stakeholder spoke on some level to this idea; almost all posed the concept in the interrogative, using the form and force of a question.

1. "Are they doing what they are supposed to be doing, getting the results that we expect and are they cost effective?"

2. "Are we using resources efficiently?"

3. "Are we getting bang for the dollar?"

4. "Are we getting good service for the dollar resources expended?"

5. "When I'm spending my money for this program, what is my valued added for the program?"

6. "Are we getting a 'return on investment?"

In most cases, these comments were connected to services to students. As one stakeholder pointed out, it is critical to stay focused on the number of students actually served by an innovation or program. Another stakeholder returned to the questioning format when he put the concern this way "what is really the direct impact on students and how do we measure it?" (p. 5). Another stakeholder said the bottom line involves doing "a heavy cost analysis on what's it costing me. If I'm worried about closing the achievement gap, I've got to know what this program is producing for me" (p. 1).

While the core focus of The School District's work is students, stakeholders acknowledged programs exist which might serve students in subsidiary capacity; one example of such a program would be a parent involvement program. Therefore, it becomes incumbent upon someone, probably the program manager in concert with the supervising director and bolstered by the full stakeholder contingency, to determine what the unit of investment actually is. The United Way calls this concept "cost per unit" 
(Budget Training Manual). A stakeholder who has served on United Way review teams as an auditor and has rich experience in this way of thinking explained, there is a "fiscal element to every program....put the numbers on the page... what does it cost us to do this program? and how many children, staff, constituents are being served by the program" (Interview, p. 4). She went on to note that effectiveness and cost per unit must be framed in terms of the definition of the unit of output so that program managers are able to articulate how many, and what type, of people they serve, with a particular amount of money to achieve a particular kind of impact. Clearly defining the scope of these particulars is one important way program managers are able to frame the work of the program and to properly filter how return on investment should be viewed, particularly by those with the discretionary power to retain or eliminate programming.

Process-use. This theme emerged multiple times throughout the interviews, despite the fact that the stakeholders did not possess precise definitions nor any professional knowledge of what Patton (1997) deems process use: the "ways in which being engaged in the processes of evaluation can be useful quite apart from the findings that may emerge from those processes" (p. 88). More specifically, it is "indicated by individual changes in thinking and behavior and program or organizational changes in procedures and culture that occur among those involved in evaluation as a result of the learning that occurs during the evaluation process" (Patton, p. 90). Given that both archival data and even a couple of comments within the electronic interview transcripts indicate that at least a couple of stakeholders believed that taking process use into account was tantamount to over-complicating things, it is interesting that the axial coding process turned up a preponderance of comments suggesting process use may well deserve 
to take center stage. Stakeholder thinking along these lines fell into roughly four categories: assailing elephants and sacred cows; increasing ownership; reifying The School District's vision, mission, goals; changing the organizational culture.

One unexpected process use which emerged was the ability of the sheer process of program evaluation, when done in depth, to assail truths or situations that have, up until that point, been ignored such as "a belief or system that is treated with much respect and is not usually criticized" (Heacock, 2003, p. 405). One stakeholder termed this "attack[ing] the sacred cows" (Interview, April 8). Another stakeholder conceived of the situation in terms of elephants and cows. His entire response, though lengthy, merits consideration.

[Program Evaluation is] a way to ferret out dysfunction.....by bringing stakeholders to the table, it kind of puts the elephant in the middle of the room and either everyone kind of testily agrees to continue not to see the Emperor has no clothes or you have to address it. And so....therefore you can either continue the culture if it's bad or you can all agree, and I think that's what would happen, is that everyone would agree. Let's address this issue and by addressing the sacred cows knock down the silo walls, increase communication, convince people that things aren't just the preserve of Mr. X because it's always been his program or her program and therefore there's a sanctity and it's inviolate...you dare not trespass into that program. I think that's one of the things, one of the good things that could come out it that might not show up in the box score necessarily. [Program evaluation] can change the culture of an organization by breaking down barriers and silos and by identifying the elephant in the room that sometimes is 
institutionally...very difficult to admit ......most programs are not that dysfunctional that there is an elephant but maybe there's a bunch of little elephants (Interview, March 20, p. 3).

Archival data supports this, and other stakeholders recognized the benefits of opening up channels of communication. The axial coding process illuminated the significance of elevating this particular process by consecrating it with a stated legitimacy; in other words, stakeholders believed there is value in getting things out into the open.

A second process use that turned up via axial coding was that of increasing ownership. One stakeholder particularly echoed the others when she noted that participating in an evaluation gave people the opportunity "to get to sit at the table and provide input" which therefore "increases level of involvement...buy-in aids in collaboration and the sense of knowing we are all in this together" (Interview, March 19, p.2). Another stakeholder explained that the process generates a feeling of validation among stakeholders at all levels within the organization- particularly for those who are not specifically in charge (p.4). He went on to say he believed it aids in building rapport within the organization and allows program managers to feel like the district is giving them information, which, in turn, generates ownership in the direction the program and the district is heading.

Ownership can be generated via voice. One stakeholder particularly emphasized the importance of validating a person's right of expression. He said it is all about having real voice and getting to use it. He categorized the benefits of this type of voice in three ways: (a) It generates belief in the program and the district (b) It creates buy-in regarding 
implementation (c) It produces the perception of something other than a top down delivery. Consequently, people with voice are able to talk and process what's happening.... "they have time to analyze it and process it and develop a plan for change" (p. 7) instead of feeling victimized or powerless in the face of it.

A third process use is the use of the program evaluation process to reify The School District's vision, mission, goals, and values. A stakeholder for this study said he believed having the program stakeholders around the table was a good opportunity to clarify how what people do fits with the stated goals. Another stakeholder commented that ideally all questions within an evaluation would "filter through [district] goals, at least looped back through them" (Interview, March 20, p. 6). Doing so would serve to reify the goals for participants, many of whom are so consumed by daily operations at the building level and have little occasion to ponder what the foundational district philosophies mean when seen in action. Yet another stakeholder argued that program evaluation should be viewed as a mechanism to constantly recalibrate back to The School District's mission of educating each child for success. A different stakeholder observed program evaluation can help participants align perspectives and expectations, not only with The School District philosophies but with those of colleagues and community.

A final process use that emerged from the individual interviews was the manner in which program evaluation can change the organizational culture. A stakeholder pointed out that program evaluation could change the culture by helping people slow down, which would help the organization become more proactive, rather than reactive. Another stakeholder commented the act itself is like putting a camera in the room: "the very fact that there is a camera there, changes behavior instantly" (Interview, March 20). 
This argument, the focus of years of research regarding the Hawthorne effect, is predicated on the notion that people change their behavior when they become the object of attention, focus, and/or study. Another stakeholder felt that the change could be most noticeable in terms of thinking practices: it could engender a solution-generating oriented mindset among participants. He explained, "program evaluation could help you reach beyond and look for solutions to problems as opposed to just evaluating what's already happening in the organization" (Interview, March 31, p. 4). A different stakeholder noted "You've got to get past the old culture sometimes to get to the new, to establish new knowledge, new thinking, expand people's knowledge base so they can develop learning on their own....this process can do that" (Interview, March 26).

\section{Electronic Survey}

In constructing the electronic survey, the Delphi technique was utilized to synthesize data and ensure all stakeholder ideas are properly represented. Garavalia and Gredler (2004) note that this "technique permits the collection of rich evaluation data" (p. 375). Particularly, it was necessary to integrate important trends illuminated during open and axial coding of the short interview transcripts. According to Garavalia and Gredler, the Delphi technique requires the researcher to find out what a group or panel of people, questioned separately think. Then their answers are summarized and rolled back out to the entire group, without being affixed to any particular individual. This technique selected by the researcher a mechanism to not only survey stakeholders, but also report interim findings to them. Additionally, the Delphi technique was selected primarily because of its potential value in overcoming geographic barriers and preserving participant anonymity -- aims of this study. 
The logic in the technique is that when people read the summaries, an activity they do alone, and are asked to comment on the ideas included within the summary, individuals within the group are more likely to legitimately unite their thinking- or conversely- legitimately diverge in thinking because they are not in the same room and subject to group think. In this case, the electronic survey (Appendix F) posed questions after reporting summarized versions of emergent themes from the Short Interviews. Eight of the nine stakeholders responded to the electronic survey. Open and Axial coding were used to make sense of the data.

Question 1. How would you define effective? Open coding of answers to this question brought to light an emphasis on purpose and pre-determined intent. Stakeholders made it clear that effectiveness could not be determined without first articulating program purpose and/or intended results. One respondent wrote, "the goals of the program must be clearly stated and understood prior to implementation. And those goals must be able to be measured. Then, the evaluation can help us understand whether we are meeting the goalsthus-is the program effective" (Electronic survey, May 2).

Question 2. What recommendation(s) do you have for balancing the need for stakeholder input against the problems associated with the routine scheduling and use of large, lengthy meetings? Responses to this question ranged from have smaller, shorter meetings to maintaining the commitment to full inclusion around the table. One respondent suggested that an early evaluative task would be to "align the level of engagement the stakeholder has with the program to the level of evaluation in which they should participate" (Electronic survey, May 5). This brought to light the concept of levels of engagement that could, as another respondent suggested, be reflected in a 
published meeting schedule. Another respondent warned, "building a culture for program evaluation will require sacrificing the ideal. Although people hate surveys, open-ended focused interviews, and large stakeholder meetings, the balance must be found among those three tools. Covey said, "No investment, no involvement" (Electronic survey, May 2). It is important to note that none of the respondents suggested doing away with large stakeholder meetings entirely. The minimum requirement seemed to be two full meetings: an orientation and a full-group wrap-up session.

Question 3. To what extent should program evaluations be designed to investigate and put a valuation on a program's qualitative benefits? Responses to this question were divided. Half of the respondents responded strongly that it was the job of program evaluation to plan for and integrate an understanding of qualitative benefits. One respondent exclaimed, "Very. Identifying and valuing (maybe not in dollars) qualitative benefits should be one of the key outputs of program evaluations. How else can we know if we are accomplishing what we intended; and it might lead to a restatement of our intent" (Electronic survey, May 3). Another wrote, "an evaluation without qualitative consideration is not complete; qualitative information is essential to see/know the whole picture" (Electronic Survey, May 2). The other half of the respondents was more noncommittal in their responses, landing squarely in the realm of "it depends" (Electronic survey, May 1). None of the respondents believed it was necessary to tie qualitative benefits to dollars.

Question 4. Should "evaluation capacity building” be an official part of the program evaluation process? Why or why not? Seven of the eight respondents said yes to this. One elaborated, "absolutely, or we'll have to do major evaluations of everything 
every five years, and, in the interim, things could go awry" (Electronic survey, May 1). Another explained, "yes, 'evaluation capacity building' is the piece that changes the culture. Without a cultural acceptance of program evaluation processes a program evaluation will not be validated by the stakeholders. It will take time to acculturate stakeholders into feeling and knowing that program evaluation is a must, not a wish" (Electronic survey, May 2). The respondent who did not respond "yes" did not exactly respond "no"-- though the response showed little patience for the concept of capacity building: "I think we are making this more complicated than it needs to be. The $\mathrm{BOE} / \mathrm{Admin}$ needs to determine which programs it wants to evaluate. Agree on the program goals, make sure they can be measured, proceed. Maybe I'm missing something here" (Electronic survey, May 2). The significance of this response is that runs contrary to the notion of stakeholder inclusion that was so roundly touted throughout the data collection. Its divergence from the mentality evidenced throughout the other data, inclusive of all electronic survey responses, indicates the fitness of the Delphi technique to allow for divergent voices. Moreover, this was not the only response that raised the question of whether or not the respondent actually understood what the question was asking. For instance, another respondent who started the response with "yes" continued with "However, I do believe that there could be a point of diminishing returns if an organization was to over evaluate" (Electronic survey, May 5).

Question 5. What process(es) or activities do you recommend to lessen anxiety on the part of the program manager whose program is about to be evaluated? Several concrete imperatives surfaced out of these answers. First, a multi-year evaluation schedule, complete with the pre-determined depth of the evaluation and what that means, 
needs to be established and published. Next, ensure program managers in-line for program evaluations are able to hear from those who have already had their programs evaluated and then be given the opportunity to pick-apart the process with the program evaluator. It is also important that the director in charge of supervising the program "blesses" the evaluation by "first affirming the [program manger] in his/her role and then by shedding light on successes already noted" (Electronic survey, May 2). In short, the program manager must be supported on a variety of cognitive and personal levels to gain a clear understanding that the evaluation is a tool for growth- "not a punitive activity" (Electronic survey, May 2).

Question 6. Please list, in order of priority from your perspective, a few examples of district PROGRAMS you believe should be evaluated within the next 24 months. With one exception, "alternative programs," the responses did not evidence a strong response pattern within the group of respondents whose answers included an array of ideas: alternative programs (6 responses); gifted (4 responses); Buchan County Children's Academy (3 responses); childcare (3 responses); social workers (2 responses); instructional coaching ( 2 responses); assessment ( 2 responses). One response regarding each of the following: summer school, class size reduction program, Title II, Appleseed grants, ESOL, tutoring, guided reading, tech instruction for teachers, GPS, PLTW (engineering and bio-med); plus any special initiatives requiring allocated funds. It is notable that the majority of these suggestions are not referenced in board policy as programs, and the converse is true. Those programs referenced in board policy as programs are noticeably absent en mass from this list. It should be noted that the following question is present because state guidelines call for evaluations of programs 
and services. Responses to the following question make it plain that stakeholders do not possess a firm understanding of the distinction between the two.

Question 7. Please list, in order of priority from your perspective, a few examples of district SERVICES you believe should be evaluated within the next 24 months. One respondent wrote, “well, I probably put a few services on screen 6 not knowing this was here" (Electronic survey, May 6). However the respondent did not go on to clarify what should have stayed on the program screen and what needed to move to the service screen. Respondents had the option of returning to screens and changing answers during the course of the survey. Another respondent seemed to view the word service as an opportunity to think further about programs as evidenced by his/her response: "see previous. I know that's too many for 24 months" (Electronic survey, May 3). The cumulative list reads: parent involvement, childcare, PAT, Homebound, 504, food services, maintenance, see previous (ie interchangeable with previous list), computer technicians, safety/emergency management plan, volunteer services (mentioned twice), business partners, employee orientation, tech support. Notably this list contains a few references to programs articulated within board policy.

\section{Second Long Interview}

The final step in the data collection sequence was a thirty-minute interview with the primary intended user. As called for in the interview protocol, the interview was preceded by a ninety-minute presentation of data collected to date. 
HIGHLGHTS OF DATA COLLECTION-

organic reporting tool

utilized prior to 2 nd long interview with primary intended user
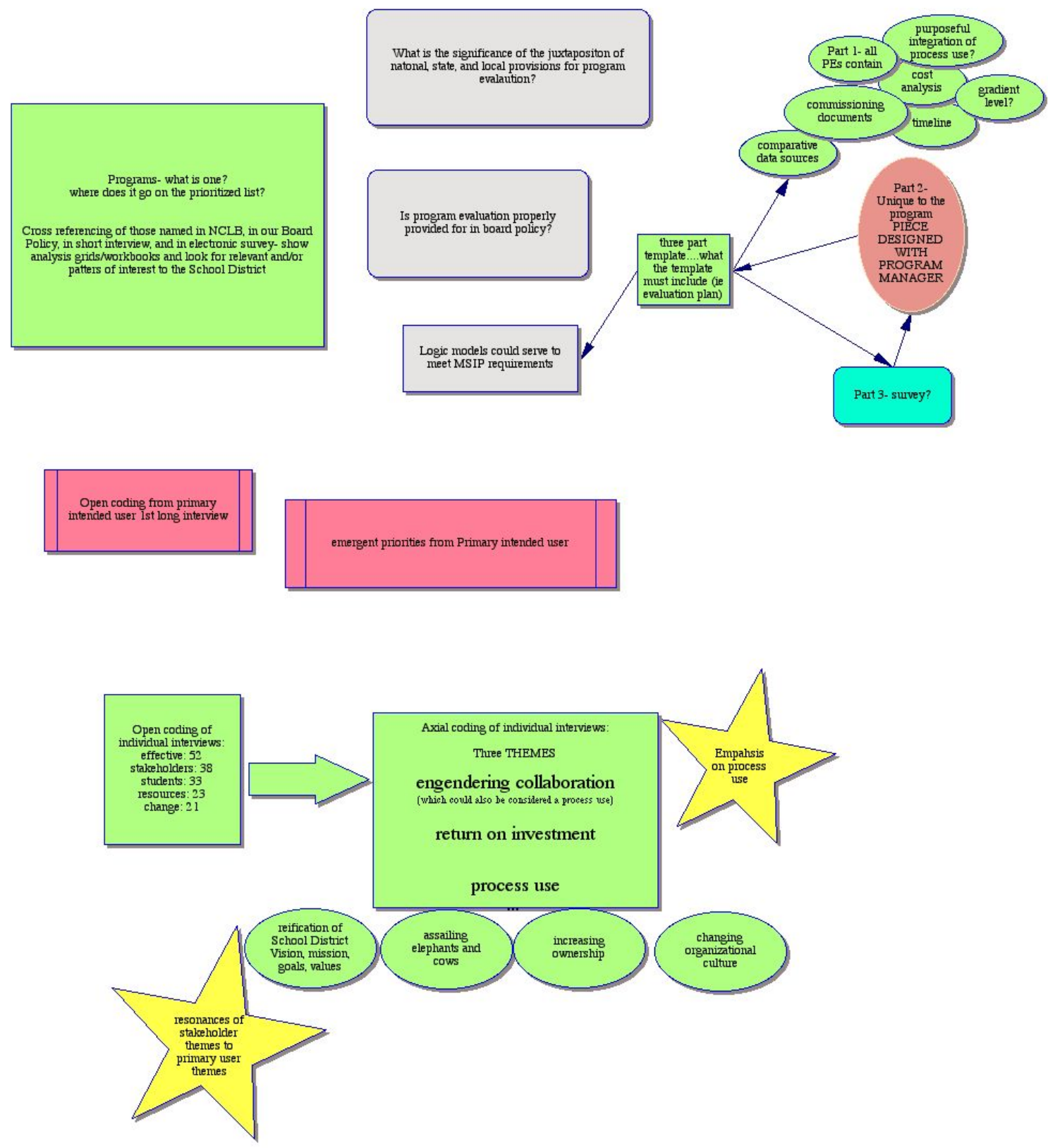

Figure 12. An organic reporting tool was utilized to facilitate second long interview. 
Again, the interview was tape-recorded, to "ensure everything said [was] preserved for analysis" (Merriam, 1998, p. 87). A research assistant not affiliated with The School District then transcribed the tape verbatim. The transcript was returned immediately upon completion to the interviewee who confirmed its accuracy; in this way, member-checking was performed.

The second long interview was preceded by a presentation of data highlights collected during the research. An organic reporting tool (Figure 12) was used to guide the presentation and ensure that important information was shared with the primary intended user. This step took ninety minutes. The interview was then driven by three questions: (a) What is your reaction to the data? (b) How do patterns within the data cause you to confirm or revise your current vision for program evaluation in The School District? (c) In terms of the organizational knowledge creation paradigm you set forth in your 2007 research, what new knowledge do you value most in this data? Why?

This interview yielded several significant concepts. "I think it is my job to pare out sacred cows and unsuccessful programming" (Interview, May 27). However, the superintendent was intrigued and slightly troubled by the summarization of programming examples (Appendix I). She furrowed her brow and said, "obviously, we need to make a list of programs and not just those that legislation stipulates we evaluate, whatever those are, but ones we believe we need to evaluate for our own reasons" (Interview, May 27).

At this point in the interview she expressed the view that The School District is about "people" not just "stuff." This point surfaced in response to her thinking regarding how her views of program evaluation have changed as a result of this study. She said that before this, her thinking was very focused upon the logistics of the UFE model. She 
explained, "that was all I knew....I don't think I really understood the people side of this" (Interview, May 27). It was this side that came out very strongly in the coding processes used upon the data. She was surprised by the significant emergence of the importance of process use, especially since these ideas came up from people who have no formal training in evaluation. Given a definition of empowerment evaluation, she responded that she could actually conceive of scenarios where such an evaluation might be commissioned, particularly given the fact that the other stakeholders in this study were responsible for the emergence of the concept of process-use. She went on to study the data reporting tool and argue that really only two main themes emerged from the stakeholders, emphasis on return on investment and emphasis on process use, because engendering collaboration is actually a process use itself. Moreover, it was very significant to her that the coding substantiated The School District commitment to the culture of collaboration.

She spoke about her own learning as a result of participating in this process and viewing the archival data from the perspective of a participant. She believes she witnessed two things: emergence of voice and emergence of leadership. She acknowledged these were unintended outcomes and wondered rhetorically, "How can you begin to put a price on that?" (Interview, May 27).

\section{Original Research Questions}

Data were collected and analyzed which resulted in the aforementioned findings that are relevant to the original research questions:

1. Can a site-specific model for program evaluation be generated? 
2. What data can be gathered to inform the creation of the program evaluation model?

3. How will data be analyzed to inform the creation of the model?

A discussion of these findings and their implications for program evaluation practice takes place in the next chapter.

Summary

These findings were the result of a Utilization-Focused Evaluation using qualitative research methods. Multiple sources of evidence were utilized to generate a program evaluation model for The School District. A discussion of these findings and their implications for program evaluation will be presented in the next chapter. 


\section{CHAPTER FIVE}

\section{DISCUSSION WITH CONCLUSIONS AND RECOMMENDATIONS}

\section{Introduction}

This research study was conducted based upon the problem that The School District had not yet determined how to implement program evaluations that are routinely capable of maximizing the use of stakeholder time, overcoming geographic constraints, and providing anonymity where necessary while still promoting organizational knowledge creation. The School District needed a program evaluation model that could balance organizational knowledge creation with cost effectiveness, efficiency, and compliance issues. The purpose of this study was to conduct a Utilization-Focused Evaluation (Patton, 1997) of The School District's program evaluation process.

Overview of the Study

The superintendent of The School District requested a Utilization-Focused Evaluation (Patton, 1997) of the district's program evaluation process. The superintendent and her council desired a cost-effective, program evaluation model that is flexible enough to provide compliance, process, causation, promotional, information while also promoting organizational knowledge creation. The researcher utilized multiple data sources, including federal, state, and local documents, archival data, current data, an electronic survey, and in-depth one-on-one interviews to satisfy the identified purpose of the evaluation.

Data analysis took place on multiple levels. Content analysis was performed upon four discrete sets of documents: The No Child Left Behind Act of 2001 (NCLB), Missouri School Improvement Program (MSIP)- Cycle 4, The School District's board 
policy, and The School District's vision, mission, and goals. Once content analysis was complete, an affinity diagram was created. Brassard (1996) notes, "this tool gathers large amounts of language data, organizes it into groupings based on the natural relationships between each item, and defines groups of items. It is a largely creative process" (p. 17).

Content analysis of archival data from The School District's program evaluation pilot was performed, and then it was analyzed utilizing an interrelationship diagraph. Again, Brassard explains, "this tool takes a central idea, issue, or problem and maps out the logical or sequential links among related items." Though it is very much like an affinity diagram, it is not the same in that "an affinity allows some initial organized creative patterns to emerge but an interrelationship diagraph lets logical patterns become apparent" (p. 41).

Open and axial coding was utilized to reduce data gathered from surveys and interviews. These processes allowed the researcher to first "fracture the data...to identify some categories, their properties, and dimensional locations" and then put the data "back together in new ways by making connections between a category and its subcategories" (Heppner \& Heppner, 2004, p. 152). In constructing the electronic survey, the Delphi technique was utilized to integrate data and ensure all stakeholder ideas were properly represented. Garavalia and Gredler (2004) note that this "technique permits the collection of rich evaluation data" (p. 375). The member check strategy was used throughout the process to provide for internal validity. 


\section{Discussion of Findings}

Three research questions guided the study. In a brief discussion of findings, the research questions are restated and discussion is based upon data analysis provided in Chapter 4. Results of analysis are provided.

\section{Research Question 1}

Can a site-specific model for program evaluation be generated? Specific tools and processes were generated as a result of this study (Figures and appendixes). It is unclear whether these constitute a model; the research indicated a necessity for the quality of fluidity that transcends the discrete processes normally conceived of within the confines of a model. However, if one agrees that the systemic use of agreed upon tools and processes constitute a simple model, then yes. A site-specific simple model for program evaluation can be generated.

During the first long interview, the primary intended user put forth the parameters for a simple model, particularly that it would have three components, one which would be standard for all evaluations, one which would be of collaborative design between the program manager and the program evaluator, and a third component, most likely the program logic model which would be submitted in advance of the evaluation. Other stakeholders were asked to describe and/or list elements they believed were necessary for inclusion and whether or not they believed the three-part structure made sense. There was unanimous agreement on the three-part structure. A chief area of emphasis relative to the components was figuring out how to provide for a structure that would allow the stakeholders of this study, who serve as the commissioning body for future program evaluations, to signify the level of involvement needed for a particular evaluation. 
Data analysis revealed that the stakeholders conceived of gradient depth of any given program evaluation in terms of the depth and breadth of stakeholder inclusion. One stakeholder explained that the depth of the evaluation could be aligned with the "level[s] of engagement of stakeholders" (Electronic survey, May 9). The idea of the program evaluation gradient (Figure 1) was modified by the researcher to integrate the intended users' conception of how a program evaluation could be rated (Figure13). All stakeholders were emailed a draft of this tool. All but one confirmed, either via email or verbally, their desire to pursue the use of this instrument. One stakeholder asked that it be applied to several hypothetical program evaluations and declared it a very utilitarian tool and suitable for immediate use. This stakeholder expressed a preference for the vertical disposition of this graphic, as opposed to the horizontal nature of the originally proposed program evaluation gradient. She noted this goes further towards expressing the concepts of depth and breadth (personal communication, June 4).

\section{Levels of Program Evaluation depth and breadth of stakeholder inclusion}

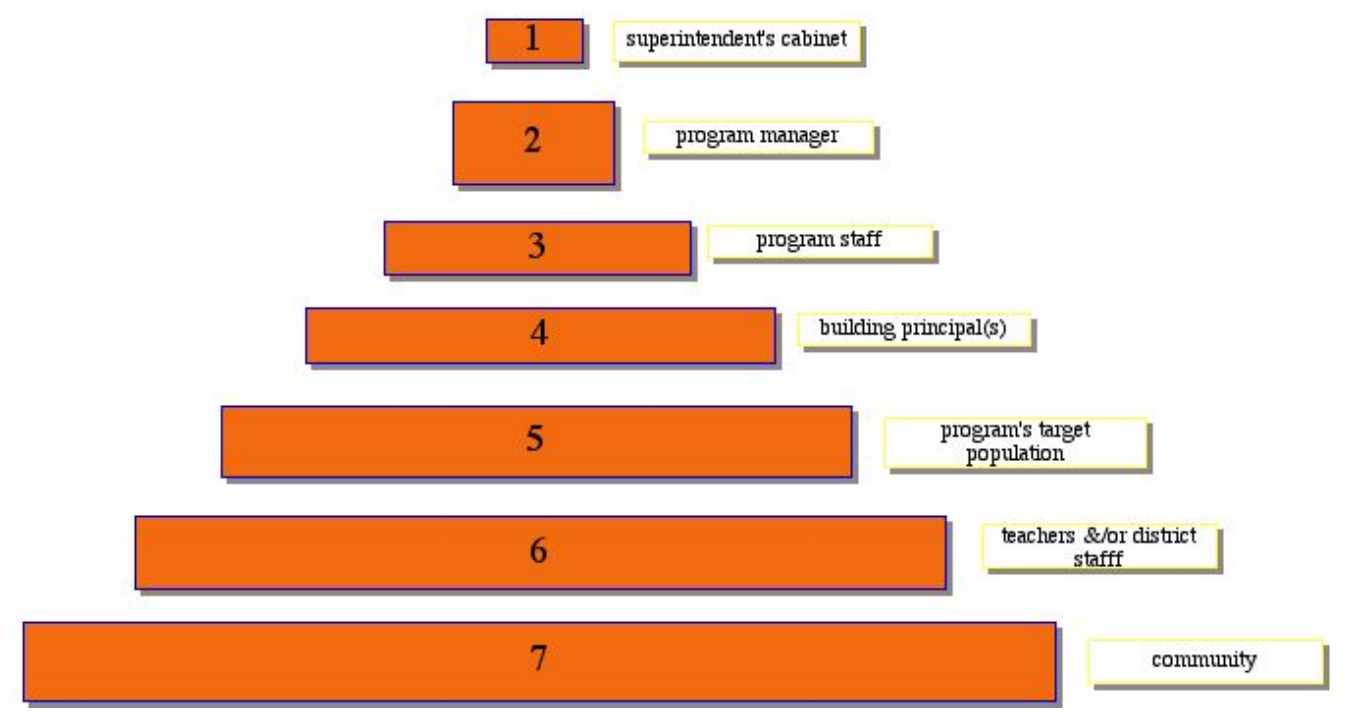


Figure 13. The level of involvement diagram provides stakeholders a tool for commissioning various gradient levels/depths of evaluations.

The Levels of Program Evaluation chart (Figure 12) is a tool that evolved from this study for use in conjunction with the program evaluation roster (Appendix R) and the program evaluation commissioning document (Appendix S). It is recommended that in addition to these tools, a program evaluation booklet be developed. This booklet should include the details of the three-part program evaluation structure, as well as the who, what, where, when, why, and how of The School District's program evaluation policy and procedures.

Research Question 2

What data can be gathered to inform the creation of the program evaluation model? Because this study was qualitative in design, data emerged from words, phrases, and narratives (Appendix P). These data gathering activities were closely aligned to the first three phases of legitimation (Figure 6). Additionally, the UFE process itself operationalized objectification (Figure 4 ) and instituationalization (Figure 5) of The School District's program evaluation philosophy/model. Research Question 3

How will data be analyzed to inform the creation of the model? Content analysis, data reduction in the form of diagrams, and coding, both open and axial, were utilized to analyze the data. From these activities, themes emerged which informed the creation of the model. However, before discussing the predominant emergent themes, it is important to note that as the research progressed, it became increasingly clear that the concept of creating a model turned out to be semi-reductive. Analysis of the data brought to light 
twin significances: procedural, easily adaptable to the types of replicatable actions typified within model construction; and the significance of process use as what Patton (2007) would deem a "sensitizing concept...as a container for capturing, holding, and examining these manifestations to better understand patterns and implications" (p. 102). The later idea is fluid, and as such is not as discrete in nature as what the study originally planned to address. Nonetheless priorities for The School District and tools for future program evaluations came about as a result of this study, even if, strictly speaking, a comprehensive, consistently replicable model did not.

Priorities originated within each theme: procedural and process use. Within the procedural theme, several priorities emerged: (a) The School District must agree upon what constitutes a program; (b) the emergent tool or model must account for cost per unit of investment and/or return on investment; (c) the emergent model must eventually include a processes/policies/philosophies handbook. It was found that failure to provide for issues related to this theme have in the past and will continue in the future to result in negative complications within the four frames of the organization: human resources, structural, political, and symbolic.

Within the process theme, five targeted benefits of program evaluation emerged:

1. Collaboration, process and results, can be engendered and promoted.

2. The School District's vision, mission, goals, and values can be reified and promoted.

3. Organizational sacred cows can be assailed.

4. Program and organizational ownership can be increased at a variety of levels. 
5. Organizational culture can be changed via knowledge creation and evaluation capacity building.

\section{Limitations}

The study contained several limitations. The study was limited because The School District is only in the first phase of professional program evaluation. The study was limited because it was based in large part upon the respondents' perceptions. The study was limited because the researcher is the research instrument. The study was limited because it is subject to interpretation and analysis by the researcher who is employed by The School District.

\section{Overall Conclusions}

Several conclusions can be drawn from this study. These conclusions can be grouped according to the two dominant themes: procedural and process use, particularly in terms of evaluative capacity building.

Procedural Conclusions. Because of the aforementioned limitations, it should come as no surprise that the study illuminated the need to implement clearly articulated processes, tools, and procedures. One very foundational conclusion, which The School District must address as soon as possible, is the idea that there is no general agreement among stakeholders regarding what constitutes a program, and therefore it is difficult for stakeholders to set program evaluation priorities. It is encouraging, however, that the analysis of programs mentioned in The School District's Board policy resonates with those types of programs mandated in NCLB. This resonance could provide The School District with a starting point for creating its definition of a program and subsequent priorities for evaluation. Again, it bears repeating that expediency in accomplishing this 
task is important if The School District expects to meet the MSIP, cycle four requirements for program evaluation. Implementing the tools created as a result of this study would be one way to come into compliance with this regulation (Appendices R \& S).

Another procedural conclusion stems from the reality that there is negligible support in The School District's board policy for program evaluation. Over the course of the past three years, The School District has made great strides in bringing its operating and philosophical procedures into alignment with board policy. The fact that there is no specific policy related to program evaluation seems to stem from the fact that The School District aligns its policy to MASA policy, which apparently has no program evaluation provision within it (personal communication, May 21).

A third procedural conclusion is that program evaluation procedures and processes must be made as transparent as possible, preferably in the form of a manual or booklet. A variety of organizational issues, which can be grouped according to Bolman and Deal's (2003) four frames, arose because program evaluation procedural issues were not properly addressed in advance of the pilot program evaluation. According to Brassard (1996) if the basic cause is solved then, a spillover effect can be expected in other areas. In this case, it is reasonable to conclude that if The School District takes comprehensive steps to shore up its program evaluation processes and procedures there will be a positive impact on human resources, organizational structure, political ramifications, and symbolic interpretation.

A fourth procedural conclusion is the notion that there should be a plainly evident mechanism for program managers to understand the role their program supervisor plays in the evaluation. Stakeholders agreed that it was the responsibility of not only program 
evaluator, but also the program supervisor as well to work with the program manager in advance of the evaluation. This is an important step in reducing evaluation anxiety, which could negatively impact the fabric of the organization if not properly addressed.

Another procedural conclusion is that the organization must continue to educate all program managers on how to construct operationalizable logic models for their individual programs in advance of the program evaluation. Archival data and interviews emphasized the significance of requiring logic models. This requirement is one way to reduce program evaluation time commitment; it also supports evaluation capacity building, addressed in the following section.

The final procedural conclusion stems from all of the others, The School District will increase knowledge creation, improve systemic thinking, build evaluative capacity, and change organizational culture by adopting an agreed upon program evaluation model, perhaps one comprised of the tools generated by this study.

Process-use conclusions. The most notable process use conclusion is that stakeholders are open to intentionally providing for process use and evaluative capacity building. Patton (1997) notes "the possibility and desirability of learning from evaluation processes as well as findings can be made intentional and purposeful. In other words, instead of treating process use as an informal offshoot, explicit and up-front attention to the potential impacts of evaluation logic and processes can increase those impacts and make them a planned purpose for undertaking the evaluation. In that way, the evaluation's overall utility is increased" (p. 88). It is important to distinguish process use from use of evaluation findings. Patton (1997) defines process use as the "purposeful application of the evaluation process to teach evaluative inquiry" (King, 2007). This 
concept also "refers to changes in attitude, thinking, and behavior that result from participating in an evaluation" (Patton, 2007). A resonance between the data for this study and accepted theory supports the conclusion that The School District is poised to begin deliberately providing for process use.

The particular, context-bound benefits of process use, as articulated by stakeholders and detailed above, are noticeably related to three of the four recognized indicators of process use: a) enhancing shared understandings; b) furthering the program intervention; c) increasing engagement; and d) program and organizational development (Patton, 1997; King, 2007; Harnar \& Preskill, 2007). It is surprising and perhaps even ironic that furthering the program intervention did not surface during the course of the study, as one might have expected given the primary intended user's concern, born out through the analysis of archival data, that there was often little evidence that people were functioning beyond the ground level (Long interview, March 18). Given this, one might have expected that the only process-use that would have emerged (furthering program intervention) was the only one that did not. 
Table Two. The research findings can be aligned to evaluation industry-wide indicators of process-use.

\begin{tabular}{|c|c|}
\hline $\begin{array}{l}4 \text { industry-wide recognized } \\
\text { indicators of process- use } \\
\text { (Patton, 1997; King, 2007; Harnar \& } \\
\text { Preskill, 2007). }\end{array}$ & $\begin{array}{l}\text { Process-use benefits emergent } \\
\text { from research with The School } \\
\text { District } \\
\text { (data analysis by researcher) }\end{array}$ \\
\hline \multicolumn{2}{|c|}{ Enhancing shared understandings $\underbrace{*}_{\text {assailed. }}$ Organizational sacred cows can be } \\
\hline \multicolumn{2}{|l|}{ Furthering program intervention } \\
\hline \multirow[t]{2}{*}{ Increasing engagement } & $\begin{array}{l}\text { * Collaboration can be engendered } \\
\text { and promoted. }\end{array}$ \\
\hline & $>^{*}$ Program ownership can be increased. \\
\hline \multirow[t]{2}{*}{$\begin{array}{l}\text { Program and organizational } \\
\text { development }\end{array}$} & $\begin{array}{l}\text { * The School District's vision, } \\
\text { mission, goals, and values can be } \\
\text { reified and promoted }\end{array}$ \\
\hline & $\begin{array}{l}* \text { Organizational culture can be } \\
\text { changed via knowledge creation and } \\
\text { evaluation capacity building }\end{array}$ \\
\hline
\end{tabular}


The resonance between the two columns is particularly intriguing given none of the stakeholders were aware of the specifically articulated nature of process-use in evaluation, nor of the prominence of those four indicators within the concept. It can be concluded that the organization is posed to leverage evaluation capacity building as a mechanism to operationalize its efforts towards achieving an even greater commitment to excellence. Doing this may ensure that high quality evaluative thinking and evaluation practices are sustainable over time.

\section{Recommendations}

Recommendations can be divided into four categories: context driven, K-12 arena, collegial and future research. These categories speak to four different audiences and canvas a wide-range of implications for individuals and organizations.

Context-bound recommendations. These stem from the procedural and process categories and are specifically bound to the context of The School District.

1. The School District should implement clearly articulated processes, tools, and procedures, such as the tools that were created as a result of this study (Figure 13, Appendix R, Appendix S). Moreover, The School District should determine whether or not it will deem the aggregate use of these tools tantamount to implementation of a model.

2. The School District should ensure MSIP, cycle four compliance and in so doing, reach a commonly held understanding of what does and does not constitute a program. 
3. The School District should complete a roster of prioritized program evaluations. This roster should include a timeline, inclusive of a provision for reporting results to the school board.

4. The School District should ensure that there is a specific board policy that provides for program evaluation.

5. The School District should make evaluation procedures and processes as transparent as possible, preferably in the form of a manual or booklet.

6. The School District should reduce evaluation anxiety by ensuring program supervisors work with program managers in advance of a program evaluation to clarify expectations.

7. Construction of a program logic model should be required in advance of any program evaluation. Program manager and staff, facilitated by program evaluation staff as necessary, should work together to do this.

8. The School District should intentionally provide for process-use as a method to deliberately provide for evaluation-capacity building within the organization.

9. The School District should consider "a small evaluation steering committee that actively manages the evaluation- capacity building process" (King, p. 50, 2007). K-12 arena recommendations. Recommendations within this section are geared toward other K-12 organizations.

1. Other K-12 organizations should include internal program evaluators within their staffing projections, if such positions do not currently exist. 
2. K-12 organizations should work together and with the American Evaluation Association to investigate and implement a professional alliance of internal K-12 program evaluators

3. K-12 organizations should look beyond the confines of federal and state program evaluation mandates to investigate the potential for intentional organizational knowledge creation.

Collegial recommendations. The following recommendations are suggested as considerations for other internal K-12 program evaluators.

1. K-12 internal program evaluators should consider utilizing the tools proposed by this research, particularly the program evaluation involvement gradient and the program evaluation-commissioning document.

2. K-12 internal program evaluators should work to ensure the procedural issues do not beget issues in other areas of the organization. One way to do this is to develop and utilize an in-house program evaluation manual.

3. K-12 internal program evaluators should study their organizations to identify "evaluation champions" who are "willing to use their positional clout to encourage involvement and model support" for program evaluation in general and evaluation capacity building activities specifically (King, p. 49, 2007). Future research recommendations. The following recommendations are suggested for further research.

1. A comparative case study should be conducted at another school district. 
2. A follow-up study of the implementation of the findings from this research. This will provide The School District additional organizational knowledge creation opportunities relative to process use of program evaluation.

3. A comparative study of other school districts that have advanced program evaluation models should be conducted. This would provide practitioners within the K-12 arena a better understanding of the long- term impact of evaluation capacity building within a K-12 school district.

\section{Summary}

The purpose of this study was to provide a Utilization-Focused Evaluation (Patton, 1997) of a Missouri School District's, hereafter referred to as The School District, program evaluation process. The distinguishing feature of the UtilizationFocused Evaluation (UFE) approach is that it emphasizes intended use by clearly identified intended users. For this UFE, the primary intended user was the superintendent of a K-12 school district in Missouri; other stakeholders for this study were identified as the highest level of leadership within The School District. This group of stakeholders is collectively referred to as the superintendent's cabinet.

The problem addressed by this study was that The School District had not yet determined how to implement program evaluations that are routinely capable of maximizing the use of stakeholder time, overcoming geographic constraints, and providing anonymity where necessary while still promoting organizational knowledge creation. The School District needed a program evaluation model that balanced organizational knowledge creation with cost effectiveness, efficiency, and compliance. 
This study employed qualitative research processes and used the social construction of reality theory as a conceptual underpinning. The use of qualitative processes allowed for the collection of rich, thick descriptions (Merriam, 1998), which was particularly important given that the social construction of reality theory is predicated upon the importance of words, phrases, and narratives to construct meaning and diffuse knowledge (Berger \& Lucckmann, 1967). Essentially, this study resulted in two types of conclusions, procedural and process-use. Procedural conclusions involve those findings that stipulate the use of specific, clearly defined steps, methods, and tools that people throughout The School District can regularly expect to encounter when participating in a program evaluation. Process-use conclusions involve those findings that hinge on Patton's (1997) notion that there of benefits of program evaluation that arise purely as a result of being engaged in the evaluation process and are "useful quite apart from the findings that may emerge from the processes" (p. 89). Of particular interest in this study is that the research findings could be aligned to three of the four evaluation industry-wide indicators of process use: enhancing shared understandings, increasing engagement, and program and organizational development (Pattton, 1997; King, 2007; Harner \& Preskill, 2007).

The most significant findings of the UFE were that a) The School District should implement clearly articulated procedures and tools, such as the type created as a result of this study, and b) The School District should also intentionally incorporate process-use within future evaluation practice as a method to deliberately provide for evaluationcapacity building and knowledge creation within the organization. 
The chief research question was whether or not a site-specific model for program evaluation could be generated. In response to the question, the study found that specific tools and procedures could be generated. By implementing these tools, procedural issues could be rectified, which would in turn impact four facets of the organization: human resources, political, symbolic, and structural. However, the research also indicated a need for fluidity in thought and practice that transcends the discrete processes normally conceived of within the confines of a model. Morgan (1997) notes "when change in the environment becomes the order of the day, as when changing technological and market conditions pose new problems and challenges, open and flexible styles of organization and management are required" (p. 44). Morgan also argues that organizations must do what they can to promote and enhance organizational memory. Clearly articulated tools and processes enhance the capacity for such memory. However, these processes cannot be held sacrosanct at the expense of the fluidity in thought and action required for organizations to not only survive - but thrive- in a constantly changing environment. 


\section{References}

AEA Task Force on Guiding Principles for Evaluators. (1995). Guiding principles for evaluators. New directions for program evaluation, Summer, pp. 19-34.

Alkin, M. C. (2004). Evaluation roots: Tracing theorists' views and influences. Thousand Oaks, CA: Sage.

Baumgartner, L. (2001). An update on transformational learning.

Merriam, S. ed. The new theory on adult learning theory. San Francisco: JosseyBass.

Berger, P. L., \& Luckmann, T. L. (1967). The social construction of reality. New York: Anchor Books.

Bolman, L. G., \& Deal, T. E. (2003). Reframing organizations: Artistry, choice, and leadership ( $2^{\text {nd }}$ ed.). San Francisco: Jossey-Bass.

Brassard, M. B. (1996). The memory jogger plus. Salem, NH: GOAL/QPC.

Bruffee, K. A. (1999). Collaborative learning: Higher education, interdependence, and the authority of knowledge $\left(2^{\text {nd }}\right.$ ed.). Baltimore: Johns Hopkins University Press.

Carden, F. \& Earl, S. (2007). Infusing evaluative thinking as process use: The case of the International Development Research Centre (IDRC). Process use in theory, research, and practice: New directions for evaluation, 116, 61-73.

Coghlan, D., \& Brannick, T. (2005). Doing action research in your organization $\left(2^{\text {nd }}\right.$ ed.). Thousand Oaks, CA: Sage.

Cronbach, L. J. (1982). Designing evaluations of educational and social programs. San Francisco: Jossey-Bass. 
Denzin, N. K. \& Lincoln, Y. S. (2005). The Sage handbook of qualitative research. Thousand Oaks, CA: Sage.

Eddy, R., \& Berry, T. (2007, November). No Child Left Behind and the discipline of evaluation: Challenges and opportunities. Paper presented at the annual meeting of the American Evaluation Association, Baltimore, MD.

Ehlers, D. C. (2004). The impact of a campus ministry based internship program on vocational choice: A case study. Dissertation (Ed.D.) University of MissouriColumbia.

Fink, A. (2006). How to conduct surveys: A step-by-step guide. Thousand Oaks, CA: Sage.

Flaherty, J. (1999). Coaching - evoking excellence in others. Burlington, MA: Butterworth-Heinemann.

Freeman, H. E. (1977). The present status of evaluation research. In Guttentag, M.A. \& Saar, S. (Eds.), Evaluation studies review annual, 2, pp. 17-51).

Fullan, M. (2001). Leading in a culture of change. San Francisco: Jossey-Bass.

Garavalia, L. \& Gredler, M. (2004). Teaching Evaluation through Modeling: Using the Delphi Technique to Assess Problems in Academic Programs. American Journal of Evaluation, 25, 375.

Hansman, C. A (2001). Context-based adult learning. Merriam, S. ed. The new theory on adult learning theory. San Francisco: Jossey-Bass.

Harner, M. A., \& Preskill, H. (2007). Evaluators' descriptions of process use: An exploratory study. Process use in theory, research, and practice: New directions for evaluation, 116, 27-44. 
Heacock, P. (2003). Cambridge international dictionary of idioms.

Cambridge, England: Cambridge University Press.

Heppner, P. P., \& Heppner, M. J. (2004). Writing and publishing your thesis, dissertation \& research: A guide for students in the helping professions. Belmont, CA: Brooks/Cole.

Horton, D. (1999). Utilization-Focused Evaluation, by Michael Q. Patton- a review. American Journal of Evaluation, 20; 399-401.

Kilgore, D. W. (2001). Critical and postmodern perspectives on adult learning. Merriam, S. ed. The new theory on adult learning theory. San Francisco: JosseyBass

King, J. A. (2007). Developing evaluation capacity through process use. Process use in theory, research, and practice: New directions for evaluation, 116, 45-59.

Kotter, J. P. (1996). Leading change. Boston: Harvard Business School Press.

Lencioni, P. (2002). The five dysfunctions of a team: A leadership fable. San Francisco: Jossey-Bass.

Marsick, V. J. \& Watkins, K. E. (2001). Informal and incidental learning. Merriam, S. ed. The new theory on adult learning theory. San Francisco: Jossey-Bass.

Merriam, S. B. (1998). Qualitative research and case study application in education. San Francisco: Jossey-Bass.

Merriam, S. B. (2001). The new update on adult learning theory. San Francisco: JosseyBass.

Morgan, G. (1997). Images of organization. Thousand Oaks, CA: Sage. 
Nelson, M. \& Eddy, R. M. (2008). Evaluative thinking and action in the classroom. In Berry, T. \& Eddy, R. M. (Eds). Consequences of No Child Left Behind for educational evaluation. New Directions for Evaluation. 117 (37-46).

Newcomer, K. E., Hatry, H. P., \& Wholey, J. S. (2004). Meeting the need for sitespecific program evaluation approaches: An introduction. In Wholey, J. S., Hatry, H. P., \& Newcomer, K. E. (Eds.), Handbook of practical program evaluation. (pp. xxxiii-xliv). San Francisco: Jossey-Bass.

New directions for program evaluation at the U.S. Department of Education. (2004, October, 15). Retrieved January 27, 2008, from http://www.ed.gov/news/pressreleases/2002/04/evaluation.html

Nonaka, I., \& Takeuchi, H. (1995). The knowledge-creating company. New York: The Oxford University Press.

Patton, M. Q. (1997). Utilization-Focused Evaluation: The new century text. Thousand Oaks, CA: Sage.

Patton, M. Q. (2007). Process use as usefulism. Process use in theory, research, and practice: New directions for evaluation, 116, 99-112.

Podems, D. (2007). Process use: A case narrative from South Africa. Process use in theory, research, and practice: New directions for evaluation, 116, 87-97.

Preskill, H., \& Donaldson, S. I. (2008). Improving the evidence base for career development programs: Making use of the evaluation profession and positive psychology movement. Advances for Developing Human Resources, 10, pp. 104-121. 
Preskill, H., \& Torres, R. (1998). Evaluative inquiry for learning in organizations. Thousand Oaks, CA: Sage.

Preskill, H., \& Torres, R. (2000). The learning dimension of evaluation use. New Directions for Evaluation, 80, 25-37.

Reason, P., \& Bradbury, H. (2001). Handbook of action research: Participative inquiry and practice. Thousand Oaks, CA: Sage.

Richardson, W. (2006). Blogs, wikis, podcasts, and other powerful web tools. New York: Corwin Press.

Rodriguez-Campos, L. (2005). Collaborative evaluations. Tamarac, FL: Llumina Press. Rossi, P. H., Lipsey, M. W., \& Freeman, H. E. (2004). Evaluation. Thousand Oaks, CA: Sage.

Russ-Eft, D., \& Preskill, H. (2001). Evaluation in organizations: A systematic approach for enhancing learning, performance and change. Boston: Perseus Books.

Scriven, M. (1996). Types of evaluations and types of evaluator. American Journal of Evaluation, 17, 151-161.

Seidman, I. (2006). Interviewing as qualitative research: A guide for researchers in education and the social sciences ( $3^{\text {rd }}$ Edition). New York, New York: Teachers College Press.

Smith. M. (2007). Psychometric properties of the school leader practice survey (SLPS) to determine Missouri school superintendent perceptions about interstate school leaders licensure consortium (ISLLC) standards performance indicators. Unpublished doctoral dissertation, (Ed.D.), University of Missouri-Columbia. 
Starratt, R. (2003). Centering educational administration- cultivating meaning, community, responsibility ( $1^{\text {st }}$ ed). Mahwah, NJ: Lawrence Erlbaum Associates.

Stufflebeam, D. L., \& Shinkflied, A. J. (2007). Evaluation theory, models, and applications. San Francisco: Jossey-Bass.

Survey monkey (2008). Retrieved May 8, 2008, from http://www.surveymonkey.com/MySurvey_EditorPage.aspx?sm=rZsLei8CEkD8S ykr3t4OSbhFbT433peN5MyOtWv0CCw\%3d

United Way, Budget Review Team Training Manual, 2008.

Wholey, J. S. (2004). Evaluability assessment. In Wholey, J. S., Hatry, H. P., \& Newcomer, K. E. (Eds.), Handbook of practical program evaluation. (pp. xxxiiixliv). San Francisco: Jossey-Bass.

Yukl, G. (2006). Leadership in organizations. Upper Saddle River, N.J.: Prentice Hall. 
Appendix A

26 Evaluation Approaches

1. Public-relations inspired study

2. Politically controlled study

3. Pandering evaluation

4. Evaluation by pretext

5. Empowerment, under the guise of evaluation

6. Objectives-based study

7. Accountability study, particularly payment-by-results

8. Success case method

9. Objective testing program

10. Outcome evaluation as value-added assessment

11. Performance testing

12. Experimental design

13. Management information system

14. Benefit-cost analysis

15. Clarification hearing

16. Case study

17. Criticism and connoisseurship

18. Program theory-based evaluation

19. Mixed-methods studies

20. Decision-and accountability-oriented study, CIPP model

21. Consumer-oriented study 
22. Accreditation and certification

23. Responsive evaluation or client-centered study

24. Constructivist evaluation

25. Deliberative democratic evaluation

26. Utilization-Focused Evaluation 


\section{Appendix B}

Permission to conduct research was requested, obtained from The School District superintendent, and then submitted to IRB. 
Appendix C

Letter of Informed Consent

\section{INFORMED CONSENT}

Researcher's Name: Laura Nelson

Researcher's Contact Information:

Work: $\operatorname{xxxxxxxxx}$

Cell: $\operatorname{xxxxxxxxxx}$

Fax: $\operatorname{xxxxxxxxxx}$

Address

City State

\section{Project Title: UTILIZATION-FOCUSED EVALUATION OF THE PROGRAM} EVALUAITON PROCESS OF A MISSOURI SCHOOL DISTRICT

To whom it may concern,

You are being asked to participate in a research study. This research is being conducted to help The School District construct a site-specific model for future program evaluations. When you are invited to participate in research, you have the right to be informed about the study procedures so that you can decide whether you want to consent to participation. This form may contain words you do not know. Please ask the researcher to explain any words or information that you do not understand.

You have the right to know what you will be asked to do so that you can decide whether or not to be in the study. Your participation is voluntary. You do not have to be in the study if you do not want to. You may refuse to be in the study and nothing will happen. If you do not want to continue to be in the study, you may stop at any time without penalty or loss of benefits to which you are otherwise entitled.

The purpose of this research is to provide a Utilization-Focused Evaluation of The School District's program evaluation process. The study will provide The School District a process and model for future program evaluations.

The study will take place over the course of approximately one month. Your participation will take place in two phases. Phase one was in the form of a one-on-one interview. You will be provided the questions in advance. The interview itself will take about twenty minutes of your time. I will contact in you in advance, via email, to set up a convenient time for me to come to your office to conduct the interview, which was tape recorded and later transcribed verbatim. Phase two was in the form of your participation in an electronic survey. It will take approximately fifteen minutes for you to complete the 
survey. In both phases you was asked to verbalize your ideas and values regarding program evaluation in The School District.

There will be nine total participants in this study. Besides you, the superintendent and the rest of the superintendent's cabinet are also being asked to participate.

Your participation will benefit the entire school district, in so far as it is anticipated a transportable, mutually understandable model for future program evaluations was the output of the study. Your participation in this study is not expected to cause you any risks greater than those encountered in everyday life.

You have the option of not participating in this study and will not be penalized for your decision.

Your identity and participation will remain confidential. Your name will not be given to anyone outside the research setting. The confidentiality of records identifying you by name was maintained. The results from the study will be published in a dissertation. The data will be stored for one year and will be destroyed one year from the publication of said dissertation.

There are no costs to you that might result from participation in this research.

It is not the policy of the University of Missouri to compensate human subjects in the event the research results in injury. The University of Missouri, in fulfilling its public responsibility, has provided medical, professional and general liability insurance for any injury in the event such injury is caused by the negligence of the University of Missouri, its faculty and staff. The University of Missouri also provides, within the limitations of the laws of the State of Missouri, facilities and medical attention to subjects who suffer injuries while participating in research projects of the University of Missouri. In the event you have suffered injury as the result of participation in this research program, you are to contact the Risk Management Officer, telephone number (573) 882-1181, at the Health Services Center, who can review the matter and provide further information. This statement is not to be construed as an admission of liability.

If you do not understand what is written above, please contact the investigator listed below.

Informed Consent is an ongoing process that requires communication between the researcher and participants. The participant should comprehend what he/she is being asked to do so that he/she can make an informed decision about whether he/she will participate in a study. To this end, you will be informed of any new information discovered during the course of this study that might influence your health, welfare, or willingness to be in this study. 
The Campus Institutional Review Board offers educational opportunities to research participants, prospective participants, the IRB process, the responsibilities of the investigator and the IRB. You may access the Campus IRB website to learn more about the human subject research process at $\mathrm{http} / / \mathrm{www}$. research.missouri.edu/cirb/index.htm.

Please feel free to contact the researcher if you have questions. Additionally, you may ask questions, voice concerns or complaints to the research advisor, Dr. Joyce Piveral.

Researcher's Contact Information:

Work: $\operatorname{xxxxxxxxx}$

Cell: $x x x x x x x x x$

Fax: $\operatorname{xxxxxxxxxx}$

Address

City State

Research Advisor's Contact Information:

Dr. Joyce Piveral

PIVERAL@,nwmissouri.edu

Phone: (660) 562-1231

Northwest Missouri State University

800 University Drive

Maryville, MO 64468-6001

The Campus Institutional Review Board approved this research study. You may contact the Campus Institutional Review Board if you have questions about your rights, concerns, complaints or comments as a research participant.

You can contact the Campus Institutional Review Board directly by telephone or email to voice or solicit any concerns, questions, input or complaints about the research study. Campus Institutional Review Board

483 McReynolds Hall

Columbia, MO 65211

573.882 .9585

e-mail: umcresearchcirb@missouri.edu

website: http://www.research.missouri.edu/cirb/index.htm

A copy of this Informed Consent form was given to you before you participated in the research.

\section{Signature}

I have read this consent form and my questions have been answered. My signature below means that I do want to be in the study. I know that I can remove myself from the study at any time without any problems. 


\section{Appendix D}

First Long Interview Protocol

\section{$\underline{\text { First Long Interview Protocol }}$}

- Conducted with The School District's Superintendent

- $\quad$ Total $=1$

- Projected Length: One hour

- Logistics: Participant was provided with informed consent

- Interview was face-to-face; researcher took notes \& tape-recorded the interview

- Recording was transcribed by research assistant

1. What is your vision for program evaluation in your school district?

2. What are the anticipated outcomes from evaluation in your mind? 


\section{Appendix E}

Short Interview Protocol

\section{$\underline{\text { Short Interview Protocol }}$}

- Place in new data collection sequence: 2

- Conducted with The School District's Superintendents' Cabinet

- $\quad$ Total $=8$

- Projected length: 20 minutes

- Logistics: Participants were provided with informed consent

- Interview was face-to-face; researcher took notes \& tape-record interviews

- Recordings were transcribed by research assistant

1. From your perspective, what is the purpose of program evaluation?

2. What common components should every district program evaluation contain?

3. In what ways can program evaluation impact culture?

4. What priorities do you have for program evaluation in the district?

5. How could or should program evaluation bring about future organizational improvement?

6. Do you have additional thoughts on the topic? 


\section{Appendix F}

\section{Electronic Survey}

- Place in new data collection sequence: 3

- Survey delivered via email to all stakeholders

- Designed, supported, and delivered via Survey Monkey

- Projected length to complete: 15 minutes

- Open-ended questions

1. During the interviews, the single word every participant said was "effective." Most participants believed the chief purpose of program evaluation was to determine whether the program being studied was "effective." How would you define "effective?" (Objectivation)

2. Stakeholder voice and/or input was viewed by participants as the second most significant factor in program evaluation. Participants identified the need to "bring stakeholders to the table." However, the ESOL pilot evaluation surfaced problems (both ideological and logistical) associated with convening large groups of stakeholders around a table on a regular basis over the course of an evaluation. What recommendation(s) do you have for balancing the need for stakeholder input against the problems associated with the routine scheduling and use of large, lengthy meetings? (Objectivation)

3. Interview transcripts reflect that each member of the Cabinet believes the benefits of program evaluation include any dollar savings, increased efficiencies or additional revenue generated. However, participants also made it very clear that it is also important to take into account qualitative benefits that relate directly to The School District's vision, mission, goals - particularly when such benefits are tied directly to students. This sentiment held true among most participants, despite the fact that often the "cost per unit" for these qualitative benefits is higher. To what extent should program evaluations be designed to investigate and put a valuation on a program's qualitative benefits?

4. Evaluation capacity building has been defined as the "intentional work to continuously create and sustain overall organizational processes that make quality evaluation and its use routine." Should "evaluation capacity building" be an official part of the program evaluation process? Why or why not?

5. The ESOL program evaluation originally did not take into account the phenomena known as "evaluation anxiety." What process(es) or activities do you recommend to lessen anxiety on the part of the program manager whose program is about to be evaluated?"

6. Please list, in order of priority from your perspective, a few examples of district PROGRAMS you believe should be evaluated within the next 24 months.

7. Please list, in order of priority from your perspective, a few examples of district SERVICES that you believe should be evaluated within the next 24 months. 
Appendix G

Second Long Interview Protocol

\section{$\underline{\text { Second Long Interview Protocol }}$}

- To be conducted with The School District's Superintendent

- Total $=1$

- Projected Length: 30 minutes

- Interview was face-to-face; researcher took notes \& taped-record interview

- Recording was transcribed by research assistant

- Prior to the start of the recorded interview, the researcher presented the superintendent with the findings to date: 90 minutes

1. What is your reaction to the data?

2. How do patterns within the data cause you to confirm or revise your current vision for program evaluation in The School District?

3. In terms of the organizational knowledge creation paradigm you set forth in your 2007 research, what new knowledge do you value most in this data? Why? 


\section{Appendix $\mathrm{H}$}

\section{Electronic Survey Cover Letter-Notice of Confidentiality}

\section{Dear Stakeholder,}

The link at the bottom of this email will take you to an electronic survey. It will take you about 15 minutes to complete it. Please know that all responses will be kept confidential. Neither your name, nor your email address was shared with anyone, inclusive of other members of the superintendent's cabinet. You will have the opportunity to view your responses and amend them, if necessary, while in the electronic environment. Other elements of the informed consent form you signed are in effect during your participation in this survey.

If you have questions or concerns, before or after taking the survey, please do not hesitate to contact me.

Sincerely, Laura Nelson, researcher

816.262.6726 


\section{Appendix I}

\section{Affinity Diagram}

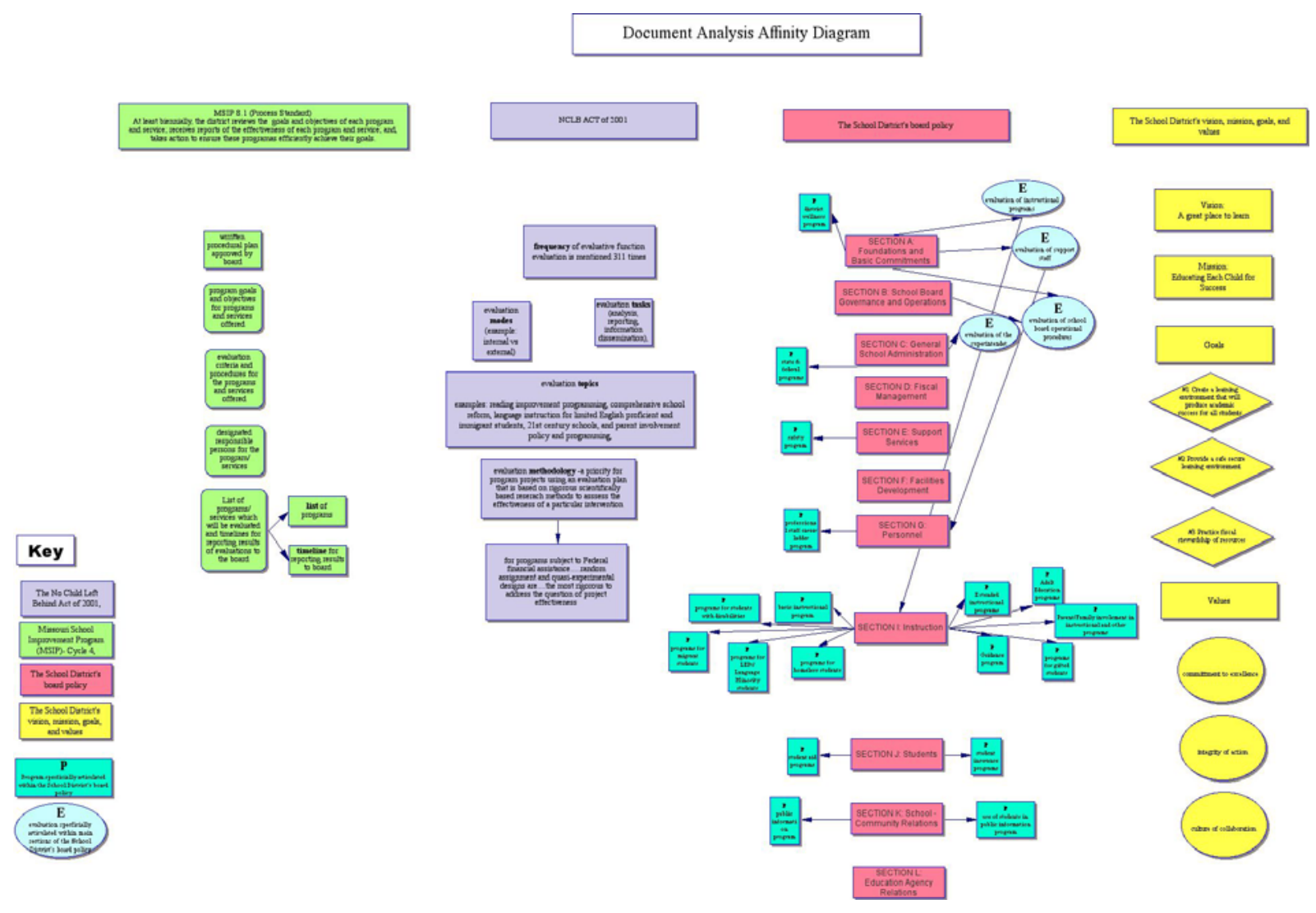




\section{Appendix $\mathbf{J}$}

MSIP, Cycle 4 Content Analysis Diagram

MSIP 8.1 (Process Standard)

At least biemnially, the district reviews the goals and objectives of each program and service; receives reports of the effectiveness of each program and service; and, takes action to ensure these programas efficiently achieve their goals.

written
procedural plan
approved by
board

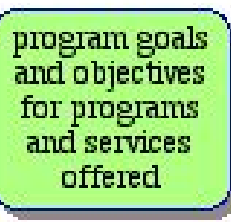

evaluation

criteria and

procedures for

the programs

and services

offered

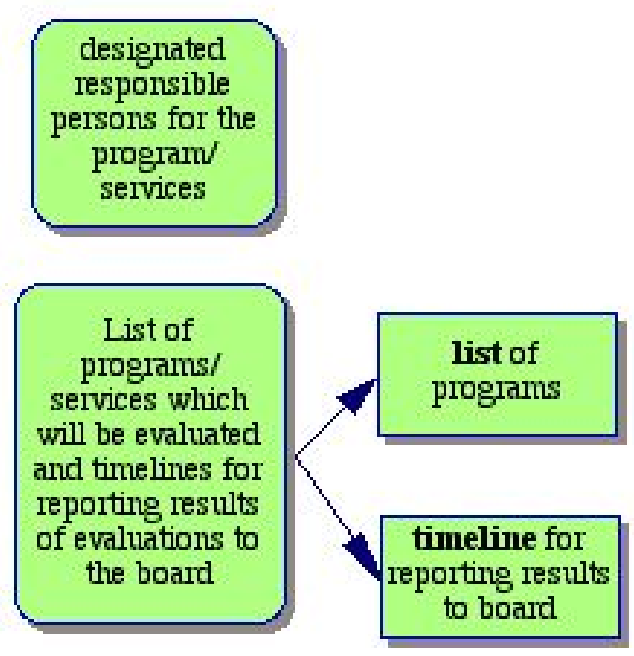




\section{Appendix K}

\section{NCLB Content Analysis Diagram}

NCLB ACT of 2001

frequency of evaluative function evaluation is mentioned 311 times

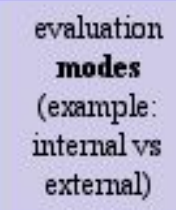

\section{evaluation topics}

examples: reading improvement programming, comprehensive school reform, language instruction for limited English proficient and immigrant students, 21 st century schools, and parent irvolvement policy and programming.

evaluation methodology -a priority for program projects using an evaluation plan that is based on rigorous scientifically based reserach methods to asssess the effectiveness of a particular intervention

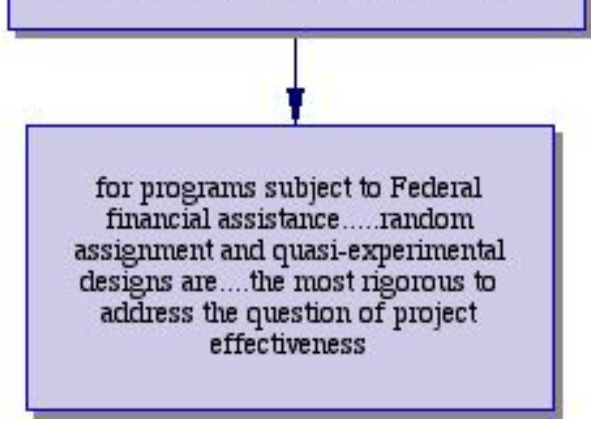




\section{Appendix L}

\section{School District Board Policy Content Analysis Diagram}

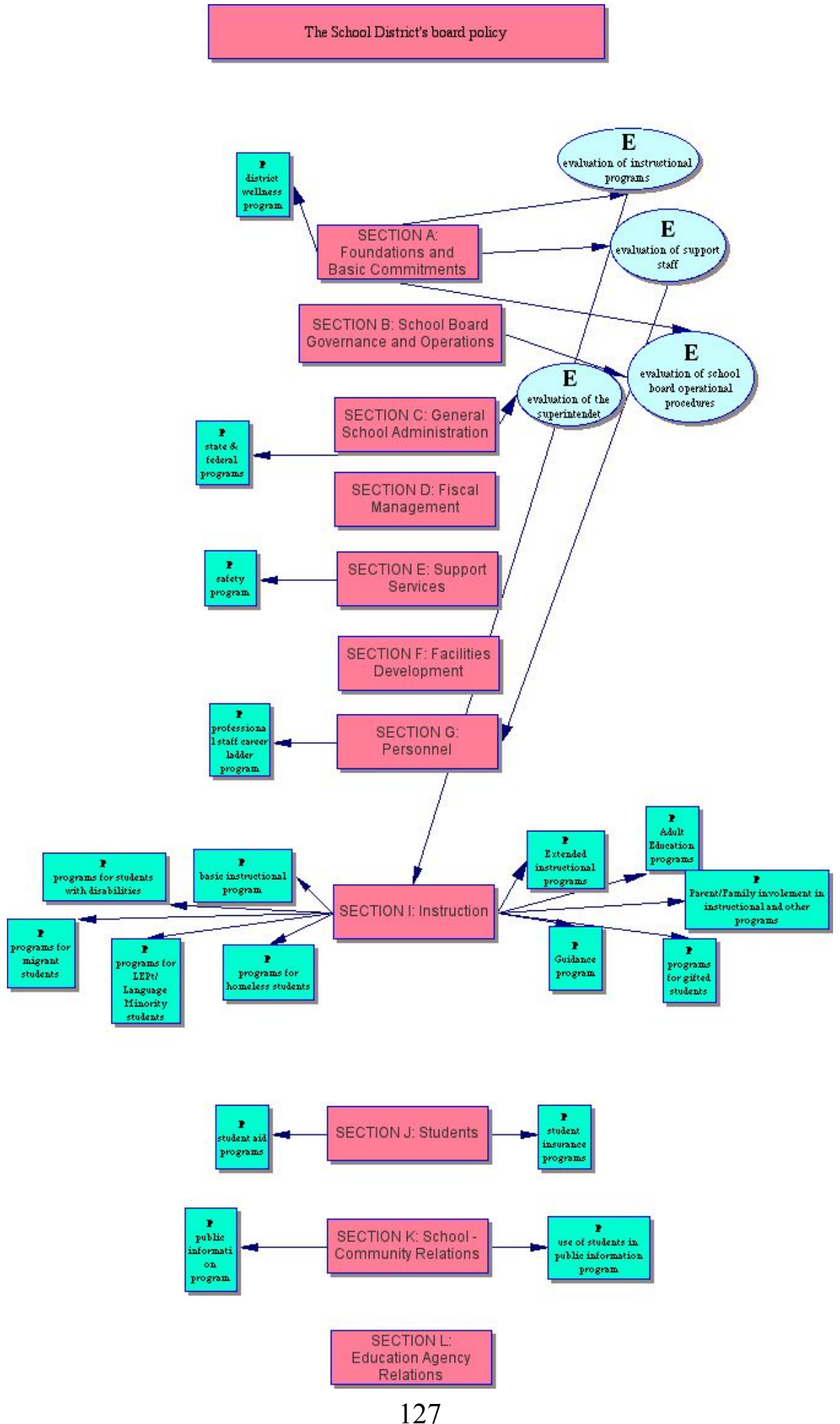




\section{Appendix M}

School District Vision, Mission, Goals and Values

The School District's vision, mission, goals, and values
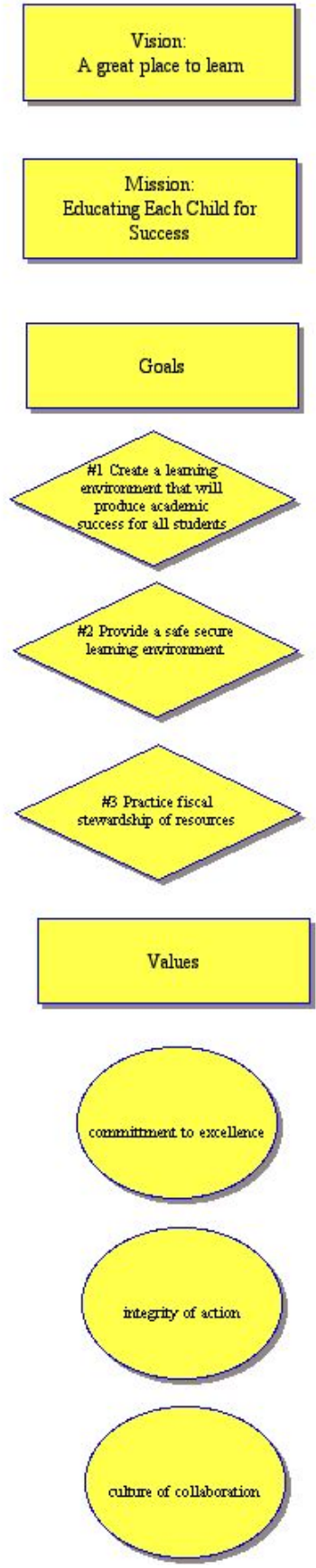


\section{Appendix N}

Example of No Child Left Behind Act of 2001 Document Analysis

\begin{tabular}{|c|c|c|}
\hline $\mathrm{Pg} / \mathrm{sec}$ & Topic & Directive \\
\hline $\begin{array}{l}77 \\
1118 \\
\text { a.2. e }\end{array}$ & $\begin{array}{l}\text { Parent } \\
\text { Involvement } \\
\text { policy }\end{array}$ & $\begin{array}{l}\text { Conduct, with the involvement of parents, an annual evaluation of the } \\
\text { content and effectiveness of the parental involvement policy in } \\
\text { improving the academic quality of the schools served under this part, } \\
\text { including identifying barriers to greater participation by parents in } \\
\text { activities authorized by this section (with particular attention to parents } \\
\text { who are economically disadvantaged, are disabled, have limited } \\
\text { English proficiency, have limited literacy, or are of any racial or ethnic } \\
\text { minority background) and use the findings of such evaluation to design } \\
\text { strategies for more effective parental involvement policies described in } \\
\text { this section. }\end{array}$ \\
\hline $\begin{array}{l}112 \\
1202 \\
\text { b.1.c }\end{array}$ & $\begin{array}{l}\text { Formula grants to } \\
\text { state educational } \\
\text { agencies }\end{array}$ & \\
\hline $\begin{array}{l}118 \\
1202 \\
\text { d.III }\end{array}$ & “ & $\begin{array}{l}\text { (info that must be recorded in a report to support the funding)The } \\
\text { progress the State educational agency and local educational agencies } \\
\text { within the State are making in reducing the number of students served } \\
\text { under this subpart in grades } 1,2,3 \text { who are reading below grade level } \\
\text { as demonstrated by such information as teacher reports and school } \\
\text { evaluations of mastery of the essential components of reading } \\
\text { instruction }\end{array}$ \\
\hline 118 & "“ & "“ “ \\
\hline $\begin{array}{l}119 \\
\sec \\
1203\end{array}$ & $\begin{array}{l}\text { State formula } \\
\text { grant applications }\end{array}$ & \\
\hline $\begin{array}{l}119 \\
b, 3\end{array}$ & & $\begin{array}{l}\text { (what the description needs to include) an assurance that the State } \\
\text { educational agency and any local LEA receiving a subgrant from that } \\
\text { State educational agency under section } 1202 \text {, will, if requested, } \\
\text { participate in the external evaluation under section } 1205\end{array}$ \\
\hline $\begin{array}{l}124 \\
\text { title } \\
1205\end{array}$ & $\begin{array}{l}\text { External } \\
\text { Evaluation }\end{array}$ & \\
\hline
\end{tabular}




\title{
Appendix O
}

\section{Examples of Programs- Data Presented to Primary Intended User}

\author{
Programs recognized by NCLB: \\ [Document Review] \\ Title 1- Improving the Academic Achievement of the Disadvantaged \\ Title II- Preparing, training, and recruiting high quality teachers and principals \\ Title III- Language Instruction for LEP and Immigrant students \\ Title IV- $21^{\text {st }}$ Century Schools \\ Title V- Promoting Informed parental choice and innovative programs \\ Title VI- Flexibility and Accountability \\ Title VII-Indian, Native Hawaiian, and Alaskan Native Education \\ Title VII- Impact Aid Program \\ Title X-part C- Homeless Education
}

\section{Programs recognized in School District Board policy: \\ [Document Review]}

District wellness program, state \& federal programs, safety program, professional staff career ladder program, programs for students with disabilities, basic instructional program, programs for migrant students, programs for LEP/LM students, programs for homeless students, extended instructional programs, adult education programs, parent/family involvement in instructional and other programs, guidance program, program for gifted students, student aid programs, student insurance programs, public information program,

\section{Programs used as examples in interviews:}

[Open Coding Interview Transcripts]

Wrestling, IB, spelling, reading recovery, boxed reading program, choir, elementary math, instructional coaching, ask principals for list of programs, parent involvement efforts, summer school, adult education programs, alternative programs, class-size reduction, new instructional practice, tutoring program, professional development program, class-size reduction guided reading

\section{List Generated from responses to electronic survey question \#6 (Please make a list of PROGRAMS you believe should be evaluated within the next two years).} [Open Coding Electronic Surveys]

alternative programs (6 responses); gifted (4 responses); BCA (3 responses); childcare (3 responses); Assessment (2 responses); Instructional coaching (2 responses); social workers (2 responses). One response regarding each of the following: summer school, class size reduction program, Title II, appleseed grants, ESOL, tutoring, guided reading, tech instruction for teachers, GPS, PLTW (engineering and bio-med); plus any special initiatives requiring allocated funds

\section{List Generated from responses to electronic survey question \#7 (Please make a list of SERVICES you believe should be evaluated within the next two years).}

[Open Coding Electronic Surveys]

Parent involvement, childcare, PAT, Homebound, 504, food services, maintenance, see previous (ie interchangeable with previous list), computer technicians, safety/emergency management plan, volunteer services (mentioned twice), business partners, employee orientation, tech support 


\section{Appendix P}

\section{Example of coded transcript}
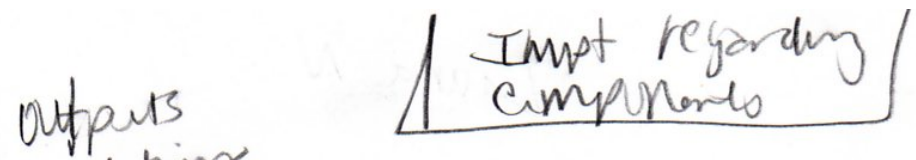

expcctution

8: I think you'd want to look at, what are the costs and what are the outputs, what are the expectations for the outputs and some way to measure how effective that is and what your cost per unit of output isto. Sounds like manufacturing but $I$ think that's still a concept that you could apply to most programs.

R: What do you mean by cost per unit of output?

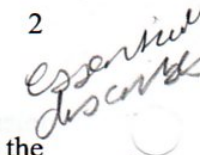

8: Well, if you have ten or fifteen kids in a program it might be cost per child served. Um. I Um. mean the child obviously isn't an output, but, it might be per
student served. Maybe that would be more like an output, but

R: Okay

9: That's what I mean

R: Okay. So you said costs, outputs, expectations, cost per unit of output. Are those the only common components you can think of? Or are there others?

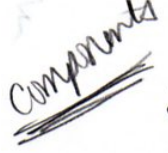
cout loud

S: Those are the ones that occur to me, quickly. Let's see, um. I would think there would be, and I'm kind of thinking reprt now, that I would expect a little bit of background so that people had a common understanding of what the program does and is expected to do. And then, of that be part of the evaluation process would be figuring out, whate the expectations because you would probably find a lot of stakeholders have different expectations.

$\mathrm{R}$ : What do you mean when you say takeholder expectations?

He:

3: With respect to differen stakeholders having different expectations, I'll use the example of the ESOL evaluation that you've just done. The teachers in that program may think the expectations are one thing, the superintendent may think something else, the business office probably has a different perspective on what those expectations are and I think you can ga on down the line, probably, with all sets of stakeholders for any program. And what they perceive the expectations to may net be the same and part of the program evaluation process maybe comes up with a demmon set of expectations. By letting those people talk to each other and find out, it may change to thectations. When you start talking to each other and find out how different they are, it may really change everybody's perspective.

R: So, I want to be clear that I got that right, so you said part of the program evaluation process might be coming up with a common set of expectations?

S: It might be, yeah

R: Or, at least -

\section{$S=$ Stakenolder coding = grean: ROI}

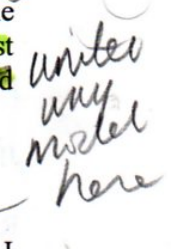




\section{Appendix Q}

Open Coding Workbook

Page 1

\begin{tabular}{|c|c|c|c|c|c|c|}
\hline Interviewee & $\begin{array}{l}\text { Effective } \\
\text { (ness) } \\
\text { (effectively } \\
\text { Ineffective) }\end{array}$ & Efficient(ly) & Stakeholders & Voice & Resource(s) & $\begin{array}{l}\text { Accountability } \\
\text { (accountable) }\end{array}$ \\
\hline Superintendent & 3 & 9 & 21 & 5 & 2 & 1 \\
\hline Assoc Super/ Dir & 1 & 1 & 1 & 0 & 1 & 4 \\
\hline Dir Information & 3 & 2 & 1 & 0 & 14 & 0 \\
\hline $\mathrm{COO}$ & 9 & 0 & 0 & 0 & 5 & 1 \\
\hline CFO & 7 & 0 & 4 & 0 & 0 & 3 \\
\hline Dir of HR & 7 & 3 & 5 & 1 & 0 & 0 \\
\hline Dir of SPED & 3 & 2 & 5 & 0 & 0 & 0 \\
\hline Asst. Dir Sec Ed & 1 & 0 & 0 & 0 & 0 & 0 \\
\hline Asst. Dir. Elem & 18 & 1 & 1 & 3 & 1 & 0 \\
\hline
\end{tabular}


Open Coding Workbook

Page 2

\begin{tabular}{|c|c|c|c|c|c|c|}
\hline Interviewee & Compliance & Opportunity & Student & Investment & Input & $\begin{array}{l}\text { Improvement } \\
\text { Improve }\end{array}$ \\
\hline Superintendent & 1 & 0 & 6 & 0 & 1 & 3 \\
\hline $\begin{array}{l}\text { Assoc Super/ Dir } \\
\text { CIA }\end{array}$ & 0 & 0 & 0 & 0 & 0 & 3 \\
\hline Dir Information & 0 & 0 & 3 & 1 & 1 & 3 \\
\hline $\mathrm{COO}$ & 0 & 0 & 7 & 3 & 0 & 1 \\
\hline $\mathrm{CFO}$ & 0 & 0 & 2 & 0 & 0 & 0 \\
\hline Dir of HR & 1 & 1 & 4 & 0 & 1 & 3 \\
\hline Dir of SPED & 1 & 0 & 2 & 0 & 0 & 0 \\
\hline Asst. Dir Sec Ed & 0 & 5 & 9 & 0 & 3 & 2 \\
\hline Asst. Dir. Elem Ed & 0 & 0 & 0 & 0 & 7 & 1 \\
\hline & 3 & 6 & 33 & 4 & 13 & 16 \\
\hline
\end{tabular}


Open Coding Workbook

Page 3

\begin{tabular}{|c|c|c|c|c|c|c|}
\hline Interviewee & $\begin{array}{l}\text { Perceive } \\
\text { perception }\end{array}$ & table & change & implementation & $\begin{array}{l}\text { Financial } \\
\text { (finance) }\end{array}$ & $\begin{array}{l}\text { Responsibility } \\
\text { (responsible) }\end{array}$ \\
\hline $\begin{array}{l}\text { Superintenden } \\
t\end{array}$ & 4 & 0 & 5 & 6 & 1 & 5 \\
\hline $\begin{array}{l}\text { Assoc Super/ } \\
\text { Dir CIA }\end{array}$ & 0 & 0 & 1 & 0 & 2 & 0 \\
\hline $\begin{array}{l}\text { Dir } \\
\text { Information }\end{array}$ & 2 & 5 & 0 & 0 & 3 & 1 \\
\hline $\mathrm{COO}$ & 0 & 1 & 3 & 0 & 1 & 0 \\
\hline CFO & 1 & 0 & 6 & 0 & 0 & 2 \\
\hline Dir of HR & 0 & 1 & 1 & 0 & 0 & 1 \\
\hline Dir of SPED & 0 & 0 & 0 & 0 & 0 & 0 \\
\hline $\begin{array}{l}\text { Asst. Dir Sec } \\
\text { Ed }\end{array}$ & 2 & 0 & 1 & 2 & 0 & 0 \\
\hline $\begin{array}{l}\text { Asst. Dir. } \\
\text { Elem Ed }\end{array}$ & 6 & 0 & 4 & 4 & 0 & 1 \\
\hline
\end{tabular}


Open Coding Workbook

Page 4

\begin{tabular}{|c|c|c|c|c|c|c|}
\hline Interviewee & value & waste & recommendations & Strength(s) & gaps & ownership \\
\hline Superintendent & 4 & 1 & 7 & 5 & 1 & 3 \\
\hline $\begin{array}{l}\text { Assoc Super/ Dir } \\
\text { CIA }\end{array}$ & 0 & 0 & 1 & 0 & 1 & 0 \\
\hline Dir Information & 4 & 2 & 4 & 1 & 6 & 0 \\
\hline $\mathrm{COO}$ & 10 & 0 & 0 & 0 & 0 & 0 \\
\hline $\mathrm{CFO}$ & 0 & 0 & 0 & 0 & 0 & 0 \\
\hline Dir of HR & 1 & 0 & 0 & 3 & 1 & 0 \\
\hline Dir of SPED & 0 & 0 & 0 & 3 & 0 & 0 \\
\hline Asst. Dir Sec Ed & 0 & 0 & 3 & 0 & 0 & 0 \\
\hline Asst. Dir. Elem Ed & 0 & 0 & 0 & 0 & 0 & 0 \\
\hline
\end{tabular}


Appendix R

2008-2010 Program Evaluation Roster

\section{The School District}

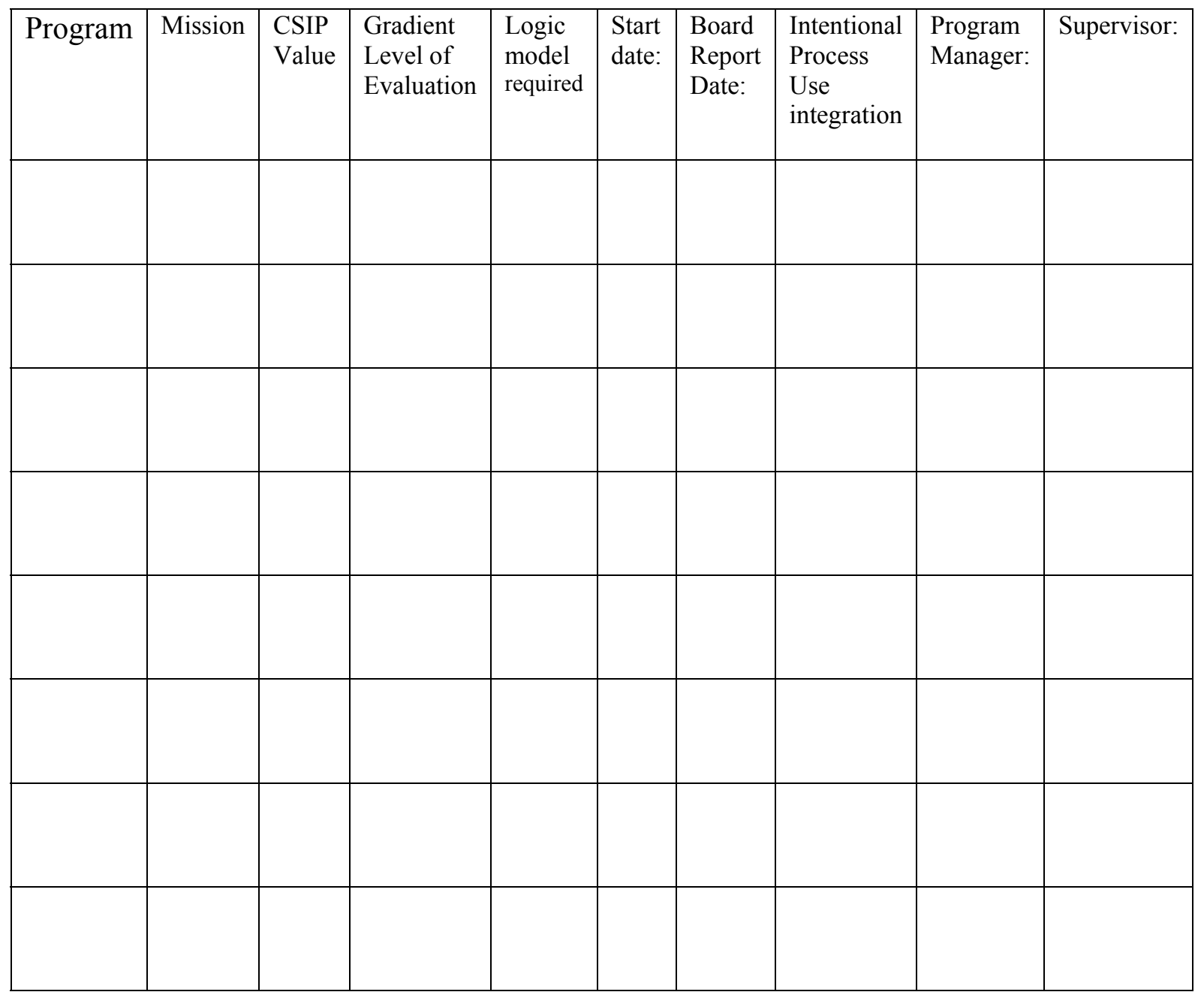




\section{Appendix S}

\section{THE SCHOOL DISTRICT PROGRAM EVALUATION COMMISSION}

Date Commissioned by Superintendent's Council:

\begin{tabular}{|l|l|l|l|l|l|l|l|l|}
\hline Program & Mission & $\begin{array}{l}\text { CSIP } \\
\text { Value }\end{array}$ & $\begin{array}{l}\text { Gradient } \\
\text { Level of } \\
\text { Evaluation }\end{array}$ & $\begin{array}{l}\text { Logic } \\
\text { model } \\
\text { required }\end{array}$ & $\begin{array}{l}\text { Start } \\
\text { date: }\end{array}$ & $\begin{array}{l}\text { Board } \\
\text { Report } \\
\text { Date: }\end{array}$ & $\begin{array}{l}\text { Program } \\
\text { Manager: }\end{array}$ & Supervisor: \\
\hline & & & $\begin{array}{l}\text { Attach to } \\
\text { this } \\
\text { document }\end{array}$ & & & & \\
\hline
\end{tabular}

Definition of Programming Unit of Service:

Payroll Expense:

Non-Payroll Expense:

Per Unit Cost of Program:

Program Goals and Objectives- expressed in terms of Outcomes and Impacts from Logic Model 
Laura Nelson earned a B.A. in Political Science from the University of MissouriKansas City, an MA in English from Northwest Missouri State University and an Ed.D in Educational Leadership and Policy analysis from the University of Missouri-Columbia. A National Board Certified language arts teacher, Laura has taught both Advanced Placement and at-risk English classes. She also served as communication arts curriculum coordinator for four years and as an instructional coach. Currently, she is the internal program evaluation analyst and assistant assessment director for the St. Joseph School District.

Laura Nelson lives in Saint Joseph, Missouri with her husband of 17 years, Tom and their children: Kit-14, Ridge-15, and Lindsey-23. Sometimes she reads for fun, but her chief hobby is finding animals in the clouds. She used to know how to cook. That was a long time ago though. 